\title{
12. RADIOLARIAN BIOSTRATIGRAPHY IN THE CENTRAL EQUATORIAL PACIFIC, DEEP SEA DRILLING PROJECT LEG 85
}

\author{
Catherine A. Nigrini ${ }^{2}$
}

\begin{abstract}
The radiolarian fauna found at the five sites drilled on Leg 85 ranges from Recent to uppermost Eocene and is both abundant and well preserved in almost all the recovered sediments. Detailed lists of 133 radiolarian events and range charts, comprising the upper and lower morphotypic limits of 73 species of Radiolaria, are presented for Sites 572, 573, 574 , and 575 .

Of the 133 events, 96 are consistently in order in the $12 \mathrm{Leg} 85$ holes. Twenty-five additional events are out of sequence in $<30 \%$ of the holes, but are still considered to be reliable and reproducible in the central equatorial Pacific. The remaining 12 events are out of sequence in $\geq 30 \%$ of the holes in which they occur. Of these, 3 are artifacts of insufficient data or coring disturbance. The remaining 9 events ( T Spirocyrtis subtilis, B Cyrtocapsella japonica, T Lychnodictyum audax, T Botryostrobus bramlettei, T Didymocyrtis laticonus, B Lithopera neotera, B Phormostichoartus corbula, B Dictyocoryne ontongensis, and B Carpocanopsis cristata s.s.) are more or less suspect for a variety of reasons.

Contrary to expectations, the percentage of out-of-sequence events, relative to the number of events tracked in various holes, is not noticeably lower in the hydraulic-piston-cored holes.
\end{abstract}

\section{INTRODUCTION}

Radiolarians were recovered from virtually all levels of the five DSDP sites drilled in the central equatorial Pacific on Leg 85 (Fig. 1). The upper portion of each site (down to maximum depth of $206.5 \mathrm{~m}$ in Hole 574) was double cored by using the hydraulic piston corer (HPC). Sites 572, 573, and 574 were then rotary drilled, with continuous coring, from a depth approximately equal to the maximum penetration of the HPC on down to basement. Site 575 was drilled with the HPC only, and basement was not reached. A single mudline core was recovered at Site 571. The locations and water depths of the sites are as follows:

\begin{tabular}{ccc}
\hline & Location & $\begin{array}{c}\text { Water } \\
\text { depth } \\
(\mathrm{m})\end{array}$ \\
\hline Site & $3^{\circ} 59.84^{\prime} \mathrm{N}, 114^{\circ} 08.53^{\prime} \mathrm{W}$ & 3962 \\
571 & $1^{\circ} 26.09^{\prime} \mathrm{N}, 113^{\circ} 50.52^{\prime} \mathrm{W}$ & 3893 \\
572 & $0^{\circ} 29.91^{\prime} \mathrm{N}, 113^{\circ} 18.57^{\prime} \mathrm{W}$ & 4301 \\
573 & $0^{\circ}$ & 4561 \\
574 & $4^{\circ} 12.52^{\prime} \mathrm{N}, 133^{\circ} 19.81^{\prime} \mathrm{W}$ & 4536 \\
575 & $5^{\circ} 51.00^{\prime} \mathrm{N}, 135^{\circ} 02.16^{\prime} \mathrm{W}$ & 4536 \\
\hline
\end{tabular}

Radiolarian assemblages range from uppermost Eocene to Recent (Fig. 2), and the fauna is both well preserved and prolific in almost all the recovered sediments.

\section{PROCEDURES}

One sample was taken from each section of core recovered during the first penetration of the HPC and the rotary drilling phase of the operation. Only core-catcher samples were taken from the second HPC penetration. Sediments were prepared in the usual manner, sieved at

\footnotetext{
${ }^{1}$ Mayer, L., Theyer, F., et al., Init. Repts. DSDP, 85: Washington (U.S. Govt. Printing Office).

${ }^{2}$ Address: 510 Papyrus Drive, La Habra Heights, CA 90631.
}

$63 \mu \mathrm{m}$, and mounted on strewn slides. For this report every corecatcher sample, and usually one intermediate sample from each core, was examined.

\section{RADIOLARIANS AT EACH SITE}

In this section, the radiolarian findings for each site are summarized. Detailed lists of events for Sites 572, 573, 574, and 575 are presented in Appendix A; range charts, plotted against depth of penetration, are presented in Figures 3 to 10. Raw data are presented in Appendix B. Unlike other DSDP reports (e.g., Westberg and Riedel, 1982), this chapter does not report abundances, but rather evaluates species on their presence or absence only. Some evolutionary transitions have been noted, but the bulk of the data presented are based on morphotypic first- and last-occurrence datum levels. In the events list, samples are designated by core and section number, and the sample depths below the seafloor are given in meters. Events and absolute ages that are inconsistent with the majority of the data collected are bracketed in the tables of events. Absolute ages are those used by the shipboard party to calculate sediment accumulation rates (see Table 1, Introduction, this volume). In the range charts, a heavy line indicates maximum confirmed range of species, and the dashed line indicates the interval between the first or last sample examined in which the species is present and the nearest sample examined in which the species is not present. An asterisk indicates a first or last occurrence that is inconsistent with the majority of the data collected. The epoch boundaries conform to those of Barron et al. (this volume). Abundances and conditions of preservation are almost always "common" and "good," so only exceptions to this general condition are noted in the text for each site.

The radiolarian zones used in this chapter for the Tertiary are those of Riedel and Sanfilippo (1978). The Quaternary zones used are those defined by Nigrini (1971). 


\section{A. NIGRINI}

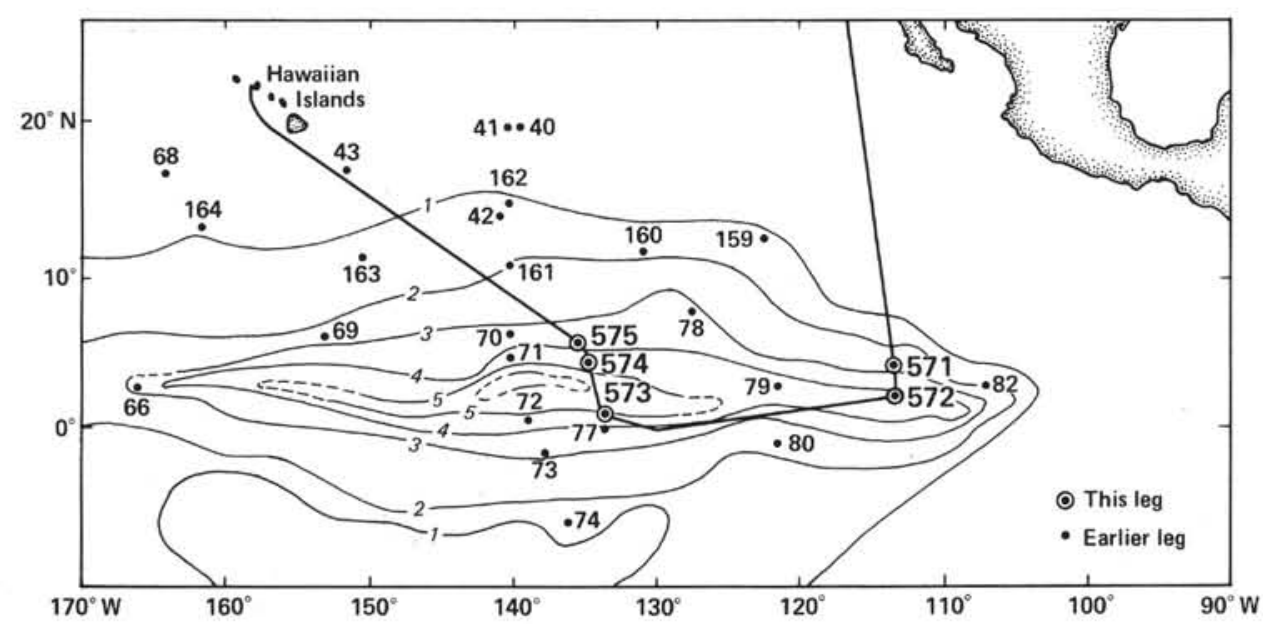

Figure 1. Location of Leg 85 and earlier drill sites. Sediment thickness is in tenths of two-way traveltime.

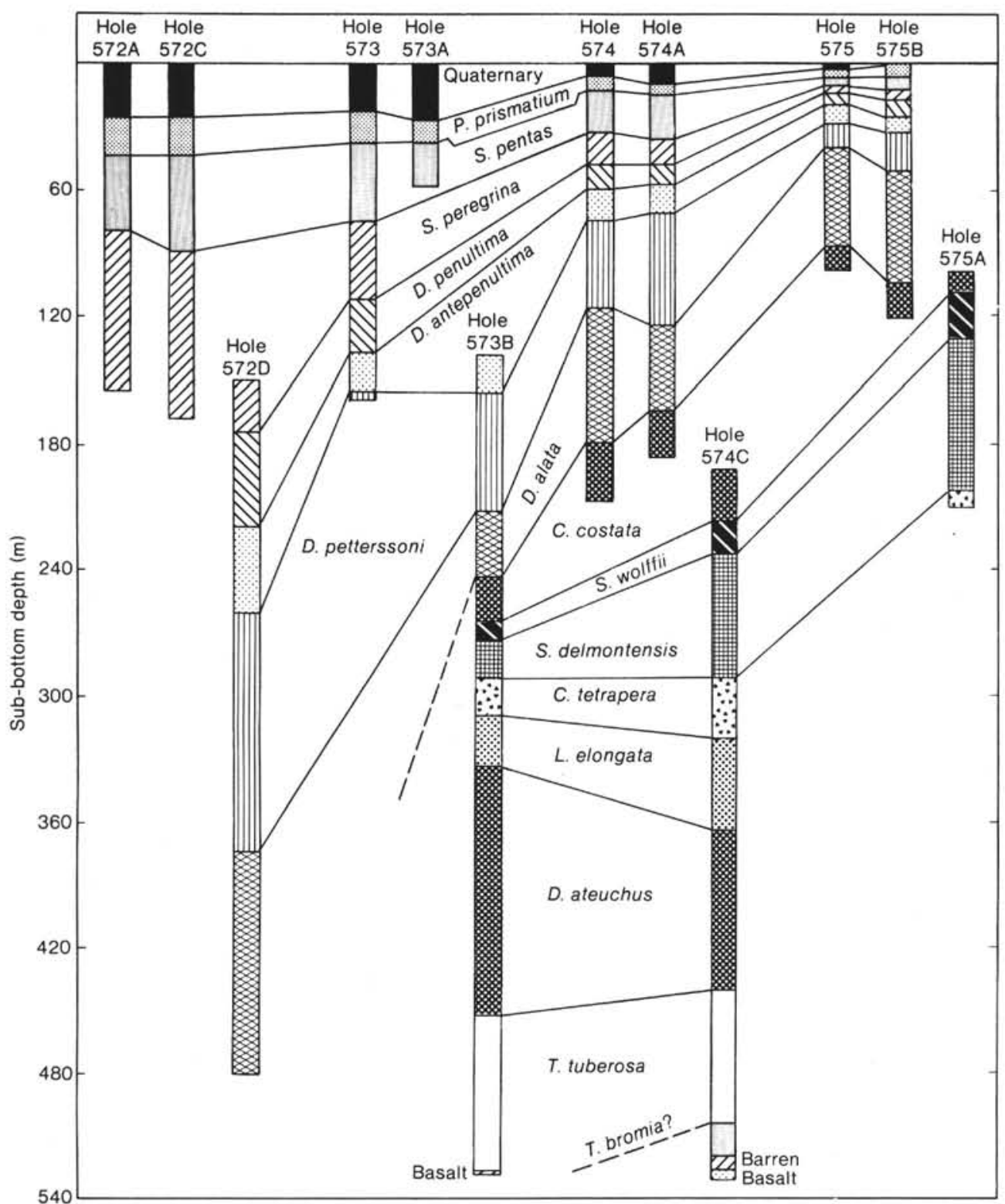

Figure 2. Summary chart of radiolarian zonation for DSDP Leg 85, Sites 572 to 575. 


\section{Site 571}

Site 571 was occupied primarily for the collection of heat-flow data. In the process, a single 7.11-m mudline core was recovered. It contains common, well-preserved, and diverse Quaternary radiolarians; the oldest sediment belongs to the Amphirhopalum ypsilon Zone (Quaternary).

\section{Site 572}

Site 572 is on the eastern edge of the equatorial highproductivity zone, slightly south and west of DSDP Site 81 . Three of the five holes drilled at this site (572A, 572C, and 572D) are sufficiently long to contain useful stratigraphic information. Holes $572 \mathrm{~A}$ and $572 \mathrm{C}$ are parallel HPC sequences, both of which end in the Stichocorys peregrina Zone (upper Miocene). Hole 572D was rota- ry drilled and continuously cored to basement $(479.5 \mathrm{~m}$ sub-bottom) and was still within the Dorcadospyris alata Zone (middle Miocene) just above the basalt. The Stichocorys peregrina Zone is unusually thick at this site, owing to a very high rate of sediment accumulation caused by an abundant upwelling diatom flora. As a result, there is some dilution of the radiolarian fauna between $572 \mathrm{~A}$ 8,CC and 572D-7,CC (72 to $218 \mathrm{~m}$ sub-bottom). A list of radiolarian events for Site 572 is presented in Appendix A, Table 1. Figures 3 and 4 are range charts for Holes 572A and 572D.

\section{Site 573}

Site 573 is near DSDP Site 77 in the eastern equatorial Pacific. Holes 573 and 573A are parallel HPC holes, but Hole 573A was abandoned prematurely. Hole 573B was rotary drilled and continuously cored to basement

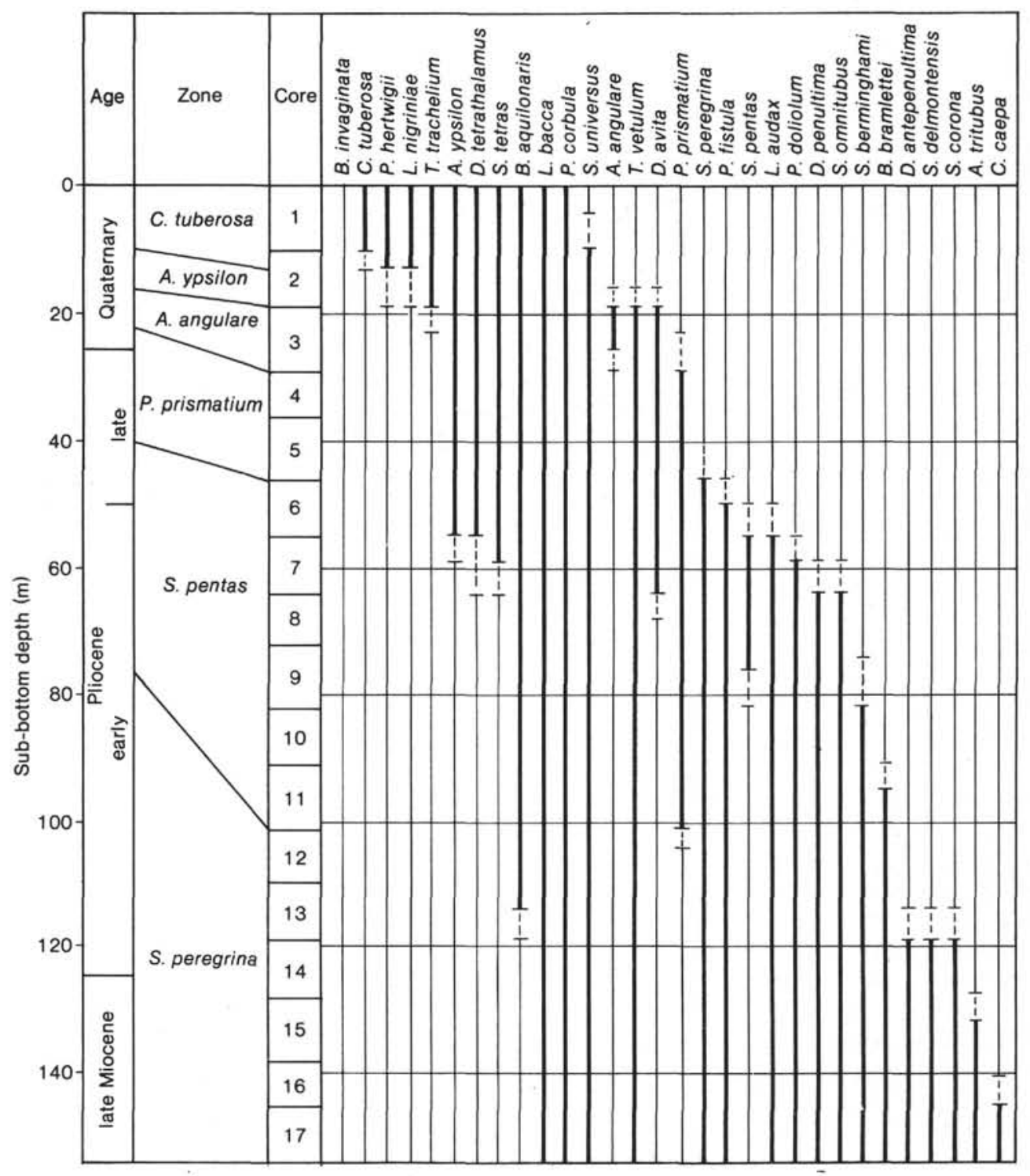

Figure 3. Radiolarian range chart for Hole 572A. Heavy vertical line indicates maximum confirmed range of a species. Dashed line interval between the first or last sample examined in which the species is present and the nearest sample examined in which the species is not present. 


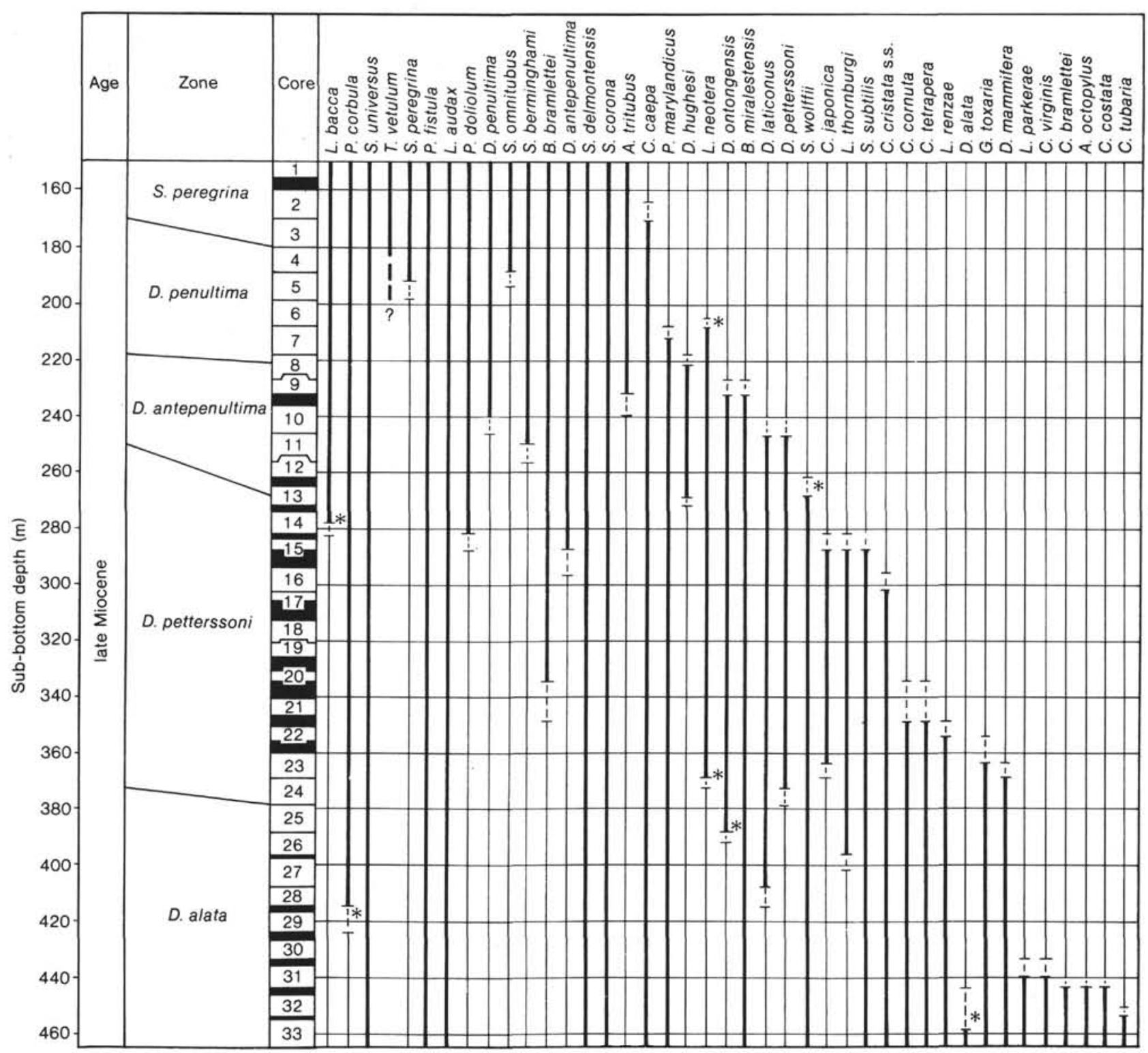

Figure 4. Radiolarian range chart for Hole 572D. Heavy vertical line indicates maximum confirmed range of a species. Dashed line interval between the first or last sample examined in which the species is present and the nearest sample examined in which the species is not present. Asterisk indicates a first or last occurrence that is inconsistent with the majority of data obtained.

(528.0 m sub-bottom). Radiolarians are common to abundant and well preserved in most of the cored sequence, which ranges from Recent to uppermost Eocene. In the lower Miocene and uppermost Oligocene of Hole 573B, however, abundance and diversity decrease, and many specimens are broken. In this interval, orosphaerid fragments and spyroid radiolarians are common. The oldest moderately well preserved radiolarian fauna is found in 573B-40, CC and is lower Oligocene (Theocyrtis tuberosa Zone) in age; an impoverished fauna belonging to the same radiolarian zone occurs in 573B-42-1, 0-1 cm, but radiolarians are absent from 573B-42-1, 149-150 cm. A list of radiolarian events for Site 573 is presented in Appendix A, Table 2. Figures 5 and 6 are range charts for Holes 573 and 573B.

\section{Site 574}

Site 574 is the second of three sites along a latitudinal transect at $133^{\circ} \mathrm{W}$ across the equatorial high-productivity belt. Hole 574 and its parallel HPC hole, 574A, bottomed in the Calocycletta costata Zone (lower Miocene). Hole 574 was rotary drilled and continuously cored to basement ( $532.5 \mathrm{~m}$ sub-bottom). Radiolarians are common and well preserved in most of the material recovered, but Core 574C-35 is barren of radiolarians.

The oldest sediments recovered are uppermost Eocene, and a good Eocene/Oligocene boundary sequence was cored. Although Hardenbol and Berggren (1978) show the Theocyrtis tuberosa/Thyrsocyrtis bromia zonal boundary to lie within P19 (lower Oligocene), most ra- 


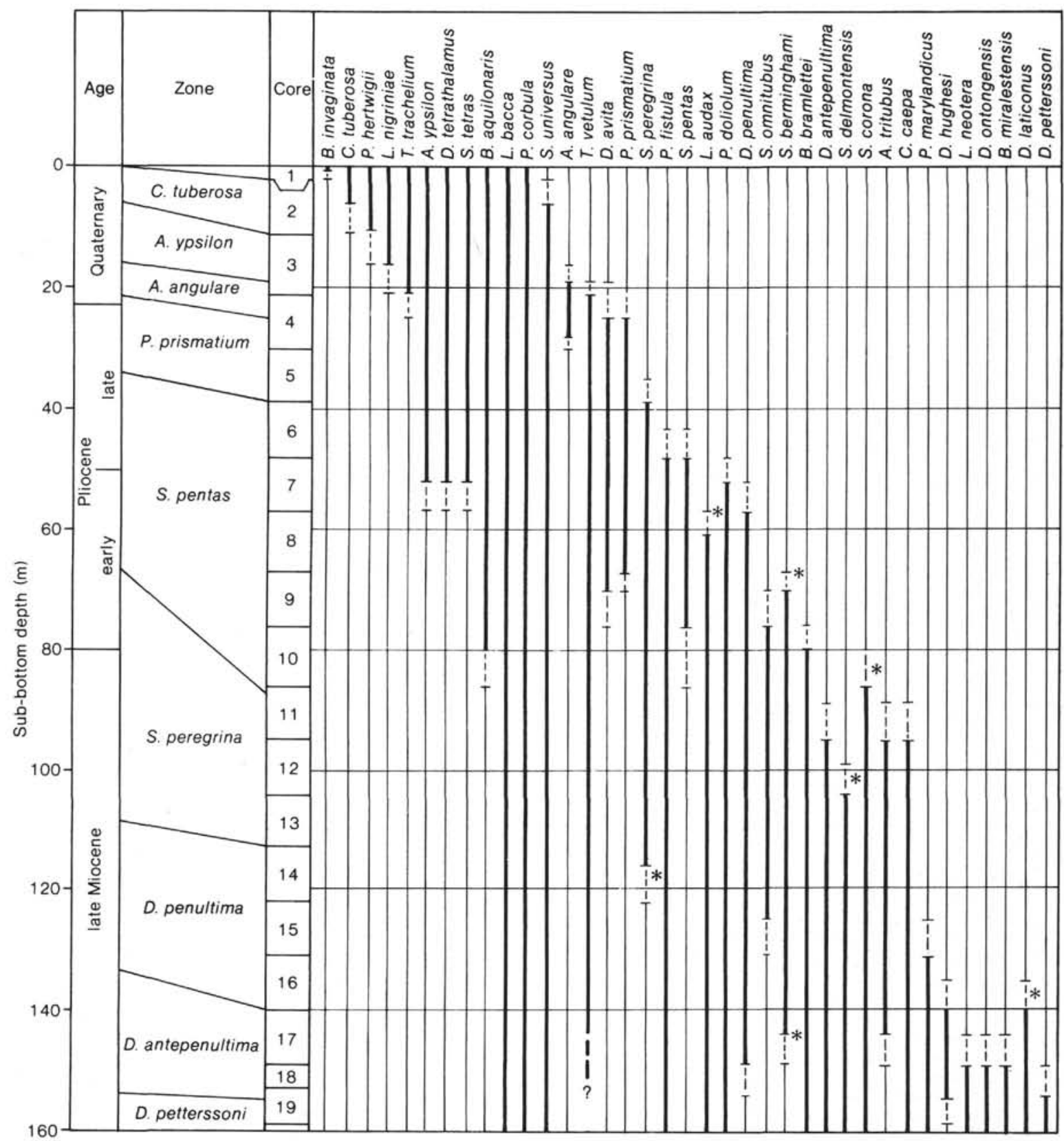

Figure 5. Radiolarian range chart for Hole 573. See caption to Figure 4 for explanation. Top, unlabeled, zone is B. invaginata Zone.

diolarian workers have, until recently, accepted Riedel and Sanfilippo's (1978) placement of that boundary as coincident with the Eocene/Oligocene boundary. Unpublished work by Riedel and Sanfilippo (personal communication, 1982) on the Bath Cliff section (Barbados) suggests, however, that a revision of the uppermost Eocene radiolarian zones is in order. Once this zonation can be tied to the foraminiferal sequence in the Bath Cliff section, and hence to the European stratotype, the Eocene/ Oligocene boundary can be precisely located with respect to the radiolarian fauna. In the present work, the boundary is tentatively placed between $574 C-33-4,49-51 \mathrm{~cm}$ (503.5 m sub-bottom) and 574C-33-5, 57-59 cm (505.08 $\mathrm{m}$ sub-bottom). A list of radiolarian events for Site 574 is presented in Appendix A, Table 3. Figures 7 and 8 are range charts for Holes 574 and $574 \mathrm{C}$.

\section{Site 575}

Site 575 is the northernmost site of a three-site transect at $133^{\circ} \mathrm{W}$. Radiolarians ranging from Recent to lower Miocene (Calocycletta costata Zone) are common and well preserved. At the top of the cored sequences (Hole 575 and its parallel HPC hole, 575B), there is considerable reworking of Miocene and Oligocene species. Quaternary and Pliocene zones are either missing or greatly compressed in the upper two cores of both holes. In addition, some drilling disturbance is indicated, making the sequence of events in these sections questionable. 


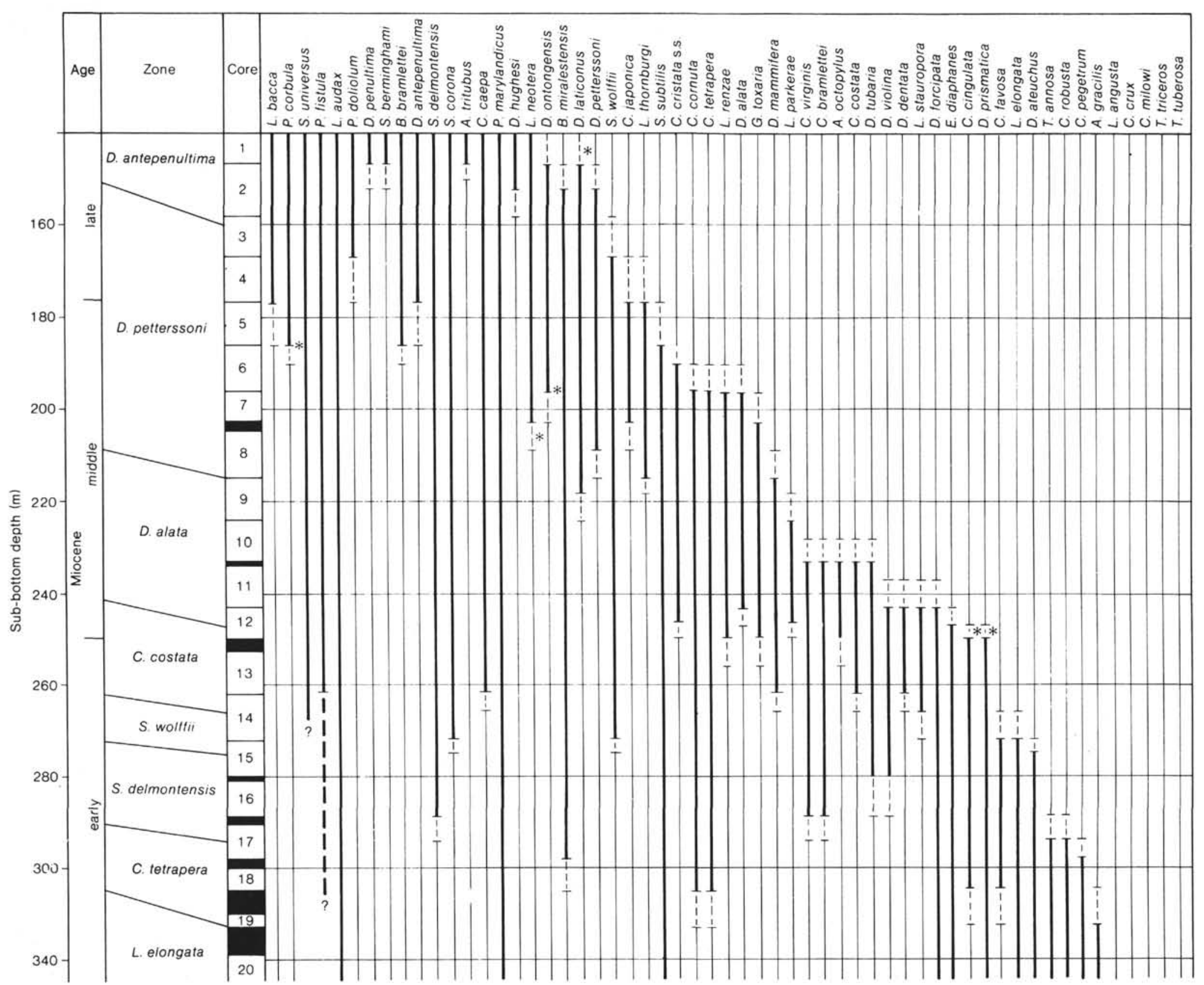




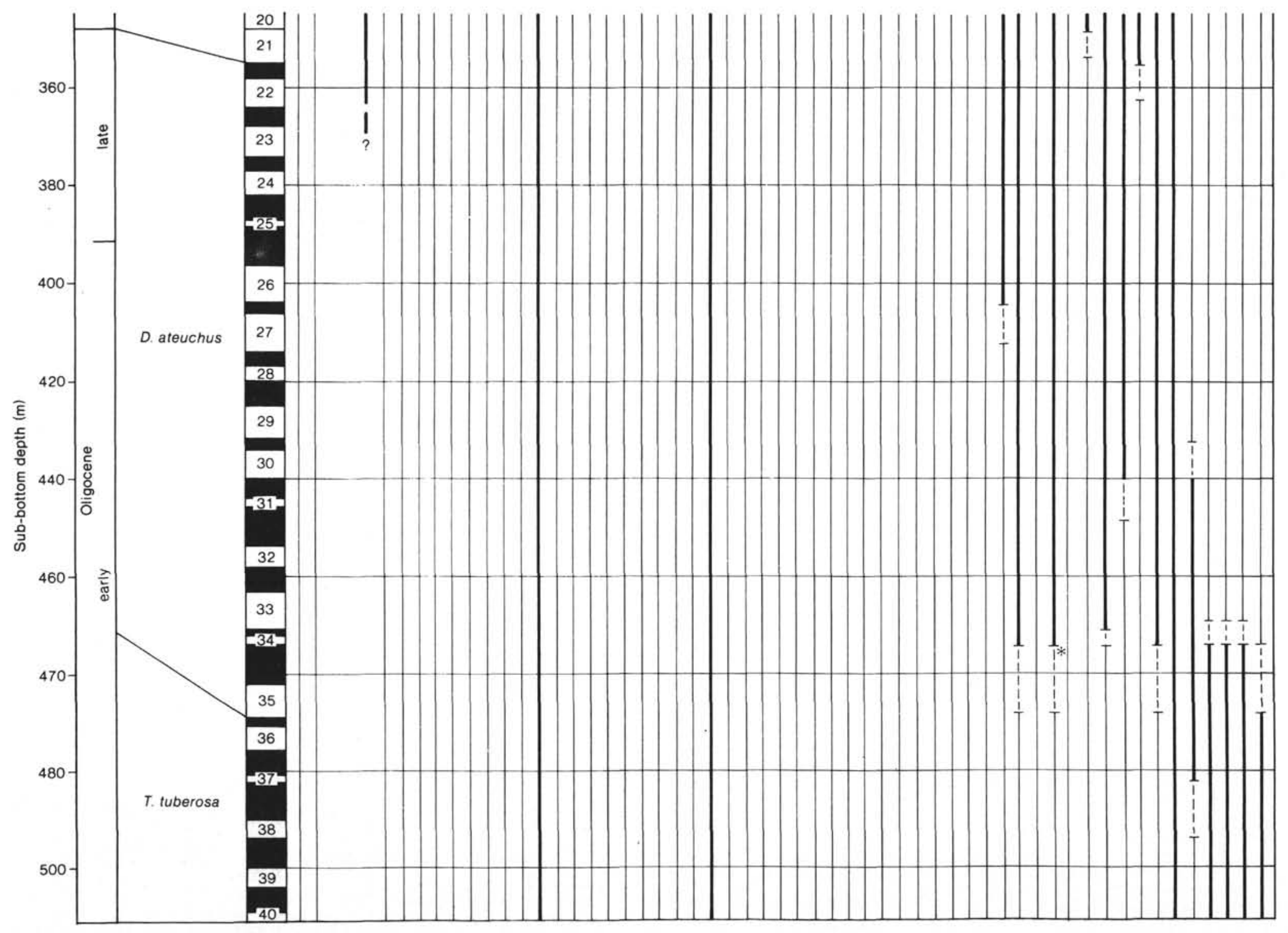

Figure 6. Radiolarian range chart for Hole 573B. See caption to Figure 4 for explanation. 


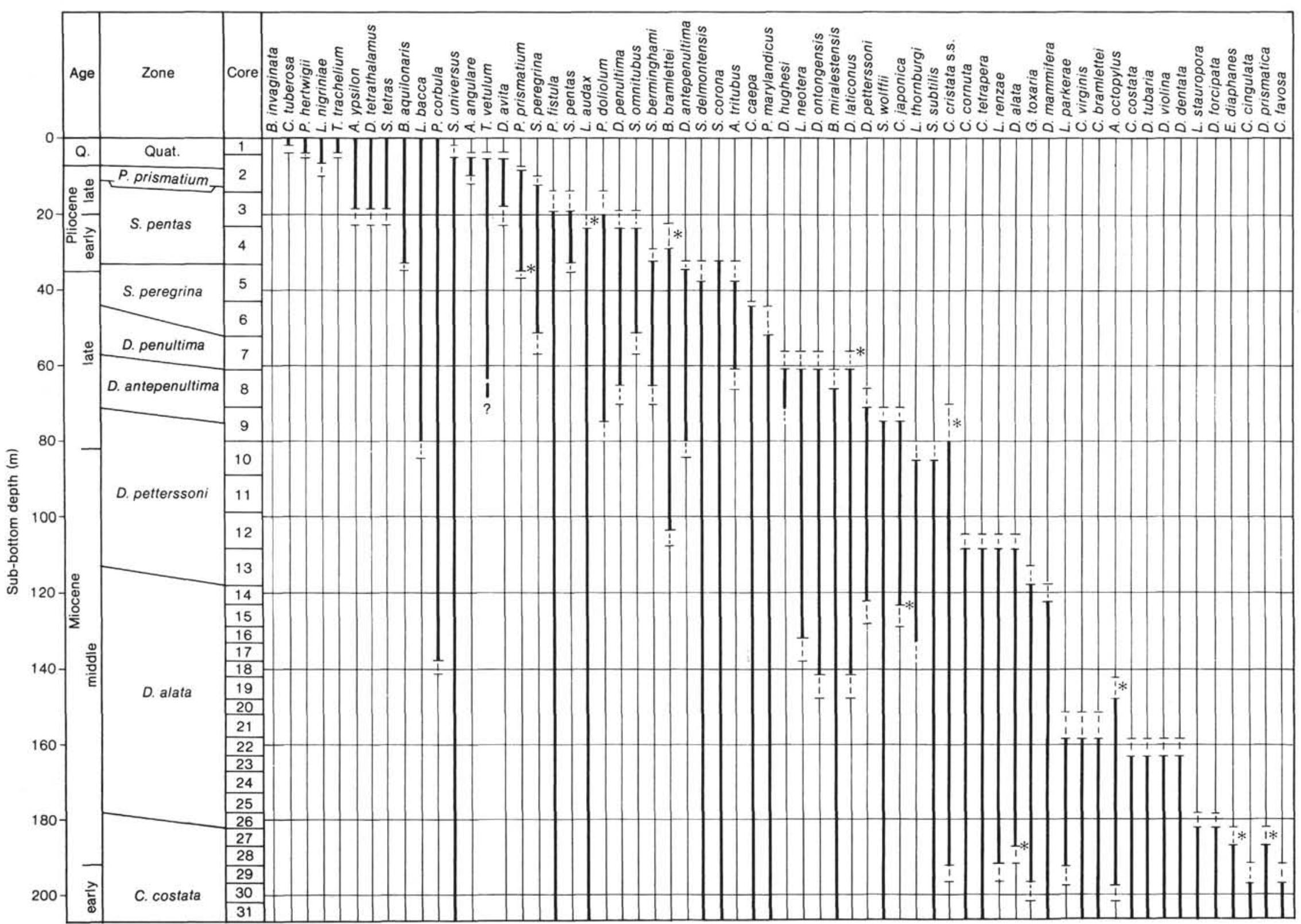

Figure 7. Radiolarian range chart for Hole 574. See caption to Figure 4 for explanation. 


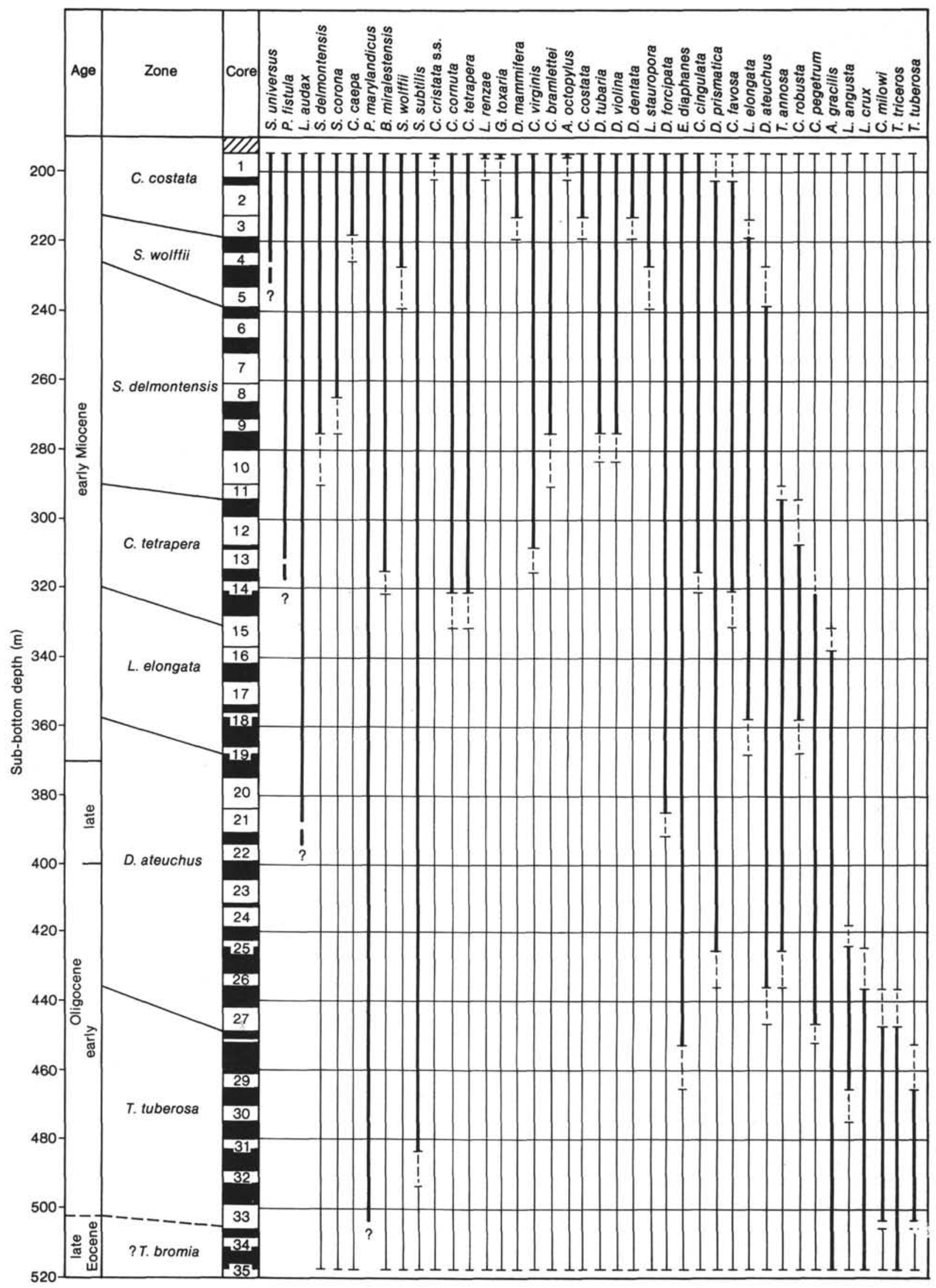

Figure 8. Radiolarian range chart for Hole 574C. See caption to Figure 3 for explanation. 
From about $20 \mathrm{~m}$ downhole, all zones are present and apparently complete. Between 575B-5,CC (48.28 m) and 575B-8,CC $(73.74 \mathrm{~m})$, radiolarian events are highly irregular (see Appendix A, Table 4). Hole 575A was continuously cored using HPC to the Cyrtocapsella tetrape$r a$ Zone (lower Miocene), but basement was not reached. See the site chapter (this volume) for a description of the coring technique used at this site. A list of radiolarian events for Site 575 is presented in Appendix A, Table 4. Figures 9 and 10 are range charts for Holes 575 and 575A.

\section{SUMMARY}

The material from DSDP Leg 85 has provided an abundant and well-preserved tropical radiolarian fauna ranging from uppermost Eocene to Recent. By using this material, it has been possible to catalogue, in considerable detail, 133 radiolarian events (primarily morphotypic tops and bottoms). The reproducibility of the sequence of these events is remarkably good, considering the close spacing of the samples. Contrary to expectations, the percentage of out-of-sequence events, relative to the number of events tracked in various holes, is not noticeably lower for the HPC holes.

Of the 133 events charted in 12 holes 37 are out of sequence in at least one hole. Of these 37 events, 25 are out of sequence in less than $30 \%$ of the holes in which the event occurs (see Table 1). A number of these events (e.g., top [T] Stichocorys wolffii, T Calocycletta virginis, T Carpocanopsis bramlettei) are only out of sequence by a single sample. Others (e.g., T Theocorythium vetulum, bottom [B] Dictyocoryne ontongensis, T Lithopera renzae) occur in a core that was found to be either greatly compressed or disturbed. Overall, it is felt that these 25 events can still be considered reliable and reproducible in the central equatorial Pacific.

Four events were out of sequence in $30 \%$ of the holes in which they occur. One of these (T Dorcadospyris ala$t a$ ) involves a disturbed core, but in Hole 572D the event is seriously out of order (by about $100 \mathrm{~m}$ ). Another of the four events ( $\mathrm{T}$ Lychnocanoma elongata) shows a high out-of-sequence percentage, but this may be an artifact of poor data, since the event occurs in only three Leg 85 holes.

The remaining eight events are out of sequence in more than $30 \%$ of the holes in which they occur. Of these eight, one (B Didymocyrtis prismatica) occurs in only two of the holes studied. Three other events (B Lithopera neotera, B Phormostichoartus corbula, B Dictyocoryne ontongensis) are out of sequence in $30 \%$ or more of the holes in which they occur, even if one discounts the fact that they are out of sequence in the section of Hole 575B that shows serious mixing of radiolarian events. These events cluster around the Diartus petterssoni/Dorcadospyris alata zonal boundary. Two events (T Lychnodictyum audax and T Botryostrobus bramlettei) fall below the $30 \%$ out-of-sequence boundary if one discounts the fact that they are out of sequence in the compressed section of Hole 574A. In general, these eight events, with the possible exception of B Didymocyrtis prismatica, cannot be considered reproducible, and are unreliable in the central equatorial Pacific either because (1) they are evolutionary events subject to errors in identification (e.g., T Didymocyrtis laticonus, B Lithopera neotera); (2) the species are rare (e.g., Dictyocoryne ontongensis); (3) the species may not be sufficiently well defined (e.g., T Lychnodictyum audax, B Carpocanopsis cristata, s.s., T Botryostrobus bramlettei); or (4) the species occur sporadically well below the level at which they are a well-established member of the faunal assemblage (e.g., B Phormostichoartus corbula).

\section{ACKNOWLEDGMENTS}

I should like to express my sincere appreciation to the Chevron Oil Field Research Company for the use of their laboratory facilities for processing all my radiolarian samples. In particular, Carlton Ford and Kevin Kirwan were helpful and pleasant laboratory companions. As usual, the drilling, marine, and technical crews of the Glomar Challenger were consistently helpful and supportive during the recovery and processing of a remarkable amount of core. Finally, my thanks also go to my family for making my participation in Leg 85 possible, and to my children for learning to make their own lunches.

\section{SPECIES LIST}

Descriptions and illustrations of the following species can be found in Nigrini and Lombari (1984).

Acrobotrys tritubus Riedel

Botryostrobus aquilonaris (Bailey)

Botryostrobus bramlettei (Campbell and Clark). Note: The upper limit of this species does not appear to be a reliable datum.

Botryostrobus miralestensis (Campbell and Clark)

Calocycletta caepa Moore. Note: The lower limit of this species is not clearly defined, because of the difficulty in distinguishing it from C. virginis when the abdominal segment is incomplete.

Calocycletta costata Riedel

Calocycletta robusta Moore

Calocycletta virginis (Haeckel)

Carpocanopsis bramlettei Riedel and Sanfilippo

Carpocanopsis cingulata Riedel and Sanfilippo

Carpocanopsis cristata (Carnevale). Note: This species is used in a restricted sense herein. Only specimens resembling those figured by Riedel and Sanfilippo, 1971, plate 1G, figure 16 and plate 2G, figure 1 , are included.

Carpocanopsis favosa (Haeckel)

Cyclampterium(?) pegetrum Sanfilippo and Riedel

Cyrtocapsella cornuta (Haeckel). Note: The upper limit of this species was found to be coincident (within the range of the sample interval) with the upper limit of $C$. tetrapera at all Leg 85 sites.

Cyrtocapsella japonica (Nakaseko). Note: The lower limit of this species appears to be an unreliable datum.

Cyrtocapsella tetrapera (Haeckel)

Diartus hughesi (Campbell and Clark)

Diartus petterssoni (Riedel and Sanfilippo)

Didymocyrtis antepenultima (Riedel and Sanfilippo)

Didymocyrtis laticonus (Riedel)

Didymocyrtis mammifera (Haeckel)

Didymocyrtis penultima (Riedel)

Didymocyrtis prismatica (Haeckel)

Didymocyrtis tubaria (Haeckel)

Didymocyrtis violina (Haeckel)

Dorcadospyris ateuchus (Ehrenberg)

Dorcadospyris dentata Haeckel

Dorcadospyris forcipata (Haeckel)

Eucyrtidium diaphanes Sanfilippo and Riedel

Liriospyris stauropora (Haeckel)

Lithopera thornburgi Sanfilippo and Riedel

Lychnocanoma elongata (Vinassa de Regny)

Lychnodictyum audax Riedel. Note: This species was not observed below the $D$. ateuchus Zone. However, Sanfilippo et al. (in press) show its lower morphotypic limit to be within the T. tuberosa Zone.

Phormostichoartus corbula (Harting). Note: The lower limit of this species was found to be an unreliable datum because of sporadic 


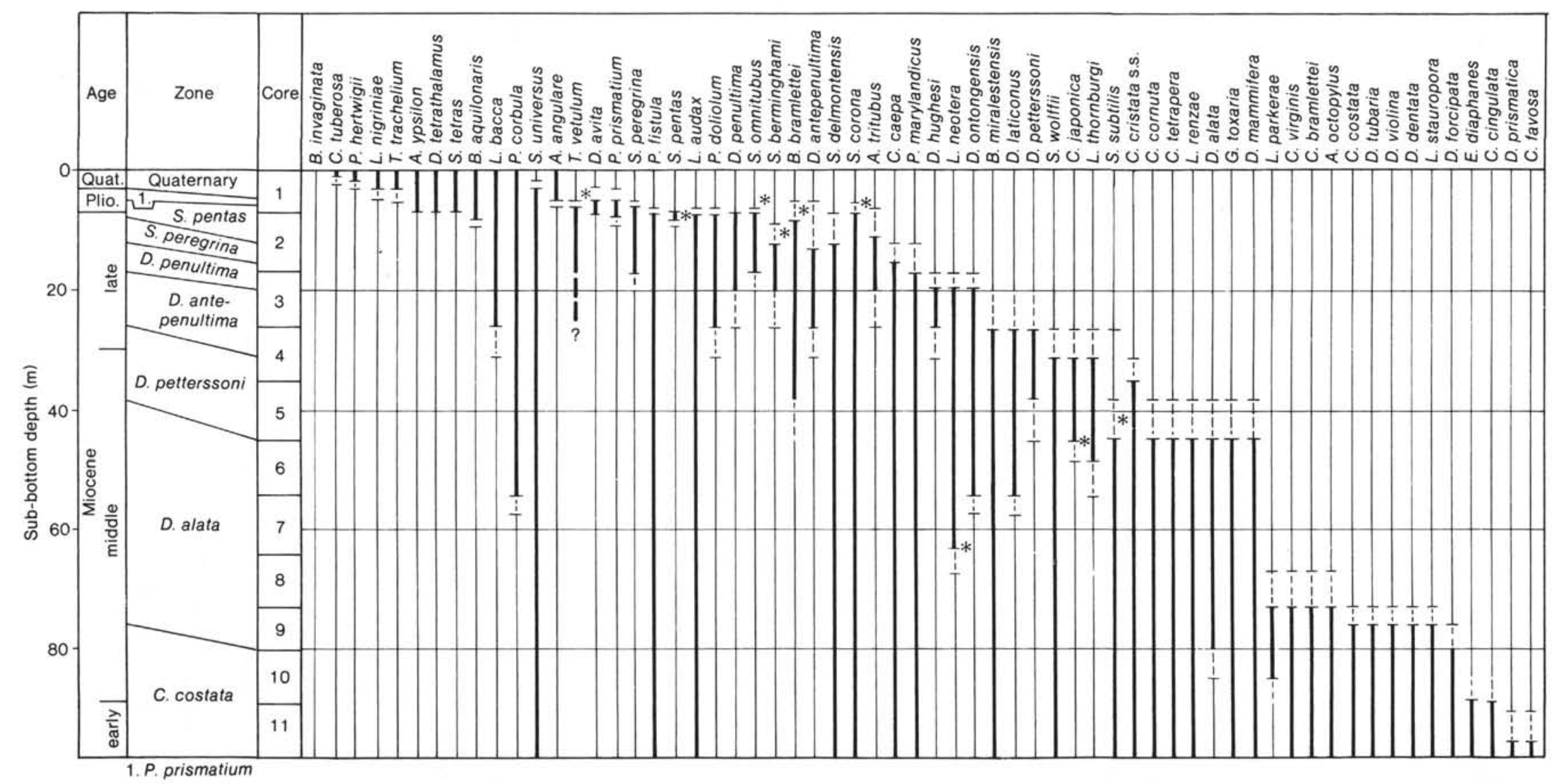

Figure 9. Radiolarian range chart for Hole 575. See caption to Figure 4 for explanation. 


\section{A. NIGRINI}

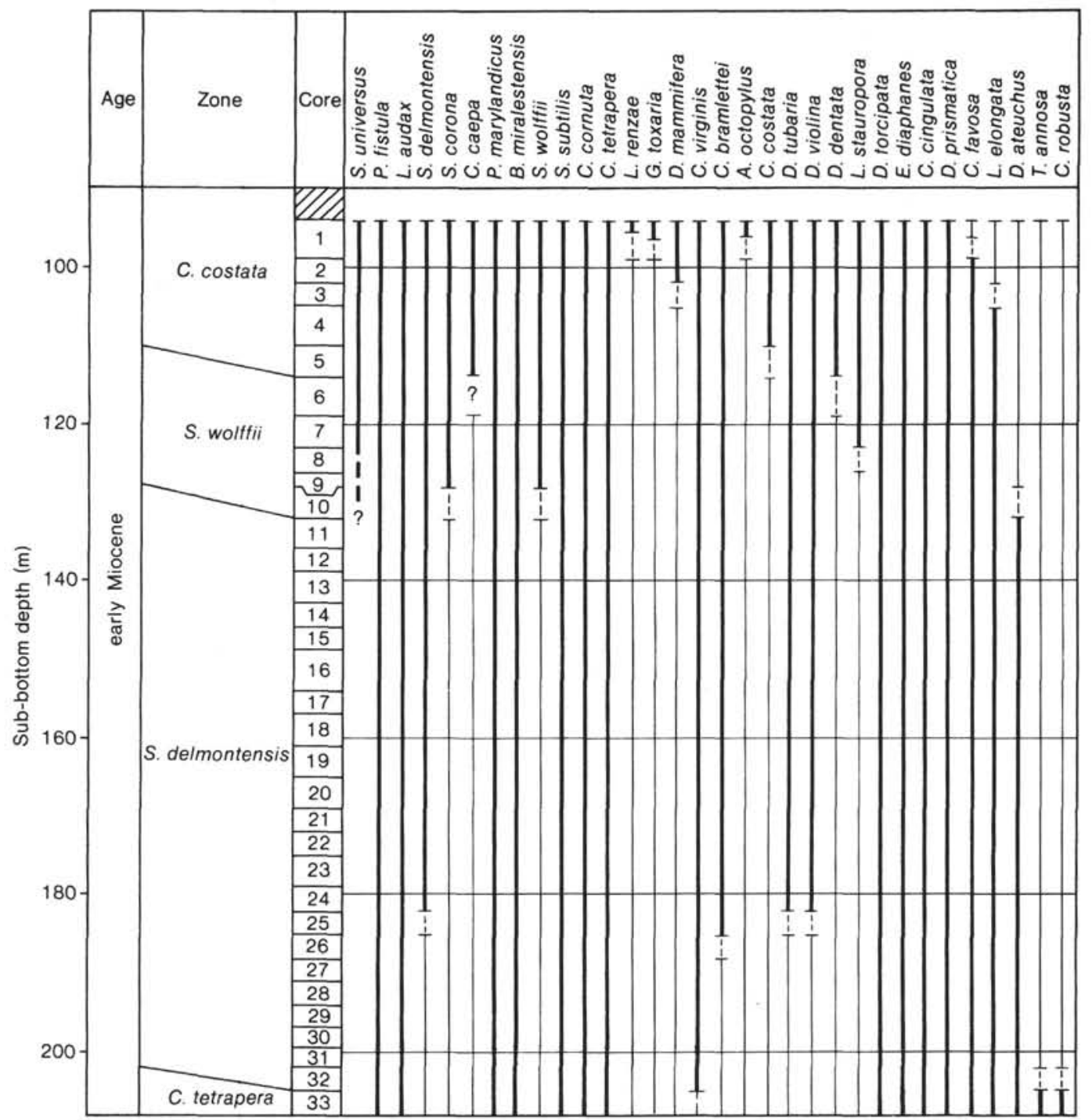

Figure 10. Radiolarıan range chart for Hole 575A. See caption to Figure 3 for explanation.

occurrences well below the level at which it is found to occur with some consistency.

Phormostichoartus doliolum (Riedel and Sanfilippo)

Phormostichoartus fistula Nigrini. Note: This species was found only sporadically below the $C$. costata Zone in Hole 573B and below the $C$. tetrapera Zone in $574 \mathrm{C}$. The lower limit could not be well defined.

Phormostichoartus marylandicus (Martin)

Pterocanium prismatium (Riedel)

Siphostichartus corona (Haeckel)

Solenosphaera omnitubus omnitubus Riedel and Sanfilippo. Note: The range of S. omnitubus procera is similar to, but somewhat narrower than, that of the nominate subspecies.

Spirocyrtis subtilis Petrushevskaya. Note: This species ranges considerably lower than was reported by Nigrini (1977).

Spongaster berminghami (Campbell and Clark). Note: Both this species and $S$. pentas are rather rare in Leg 85 sediments, making definition of the $S$. peregrina/S. pentas zonal boundary difficult.

Spongaster pentas (Riedel and Sanfilippo)

Stichocorys delmontensis (Campbell and Clark)

Stichocorys peregrina (Riedel)

Stichocorys wolffii (Haeckel)

Stylatractus universus Hays (= Axoprunum angelinum). Note: The lower limit of this species is not defined herein. Specimens are found in sediments at least as old as the $S$. wolffii Zone.
Theocorythium vetulum Nigrini. Note: The lower limit of this species is not defined herein, because the relationship between it and various species of Lamprocyclas is not presently understood.

Theocyrtis annosa (Riedel)

Descriptions and illustrations of the following species can be found in Nigrini and Moore (1979).

Amphirhopalum ypsilon Haeckel

Collosphaera tuberosa Haeckel

Didymocyrtis tetrathalamus (Haeckel) (= Ommatartus tetrathalamus)

Lamprocyrtis nigriniae (Caulet). Note: The closely related species $L$. heteroporus and $L$. neoheteroporus were found to be unreliable stratigraphic markers in the latitudes covered by Leg 85 .

Peterocorys hertwigii (Haeckel)

Spongaster tetras Ehrenberg

Theocorythium trachelium (Ehrenberg)

Descriptions and illustrations of the following species may be found in the publications cited.

Acrocubus octopylus Haeckel; Haeckel, 1887; Goll, 1972

Anthocyrtidium angulare Nigrini; Nigrini, 1971

Artophormis gracilis (Riedel), Riedel, 1959; Riedel and Sanfilippo, 1970

Buccinosphaera invaginata Haeckel; Nigrini, 1971, and Knoll and Johnson, 1975 
Table 1. Summary of out-of-sequence radiolarian events.

\begin{tabular}{|c|c|c|c|c|c|c|c|c|}
\hline & \multirow[b]{2}{*}{$\begin{array}{c}\text { Radiolarian } \\
\text { event }^{\mathrm{a}}\end{array}$} & \multicolumn{4}{|c|}{ Out of sequence } & \multicolumn{3}{|c|}{ Possible explanation } \\
\hline & & Frequency & Percentage & $\begin{array}{c}\text { By 1 } \\
\text { sample }\end{array}$ & $\begin{array}{l}\text { By }>1 \\
\text { sample }\end{array}$ & $\begin{array}{c}\text { Rare } \\
\text { species }\end{array}$ & $\begin{array}{c}\text { Member of } \\
\text { evolutionary } \\
\text { sequence }\end{array}$ & $\begin{array}{l}\text { Disturbed or } \\
\text { compressed } \\
\text { core }\end{array}$ \\
\hline $\mathrm{T}$ & Theocorythium vetulum & 1 in 7 & 14 & $\sim$ & & & $r$ & $\sim(575)$ \\
\hline $\mathrm{T}$ & Phormostichoartus fistula & 1 in 8 & 12 & $\checkmark$ & & & & $\sim(574 \mathrm{~A})$ \\
\hline $\mathrm{T}$ & Spongaster pentas & 2 in 8 & 25 & $\sim$ & $\boldsymbol{r}$ & $\checkmark$ & $r$ & $\checkmark(575)$ \\
\hline $\mathbf{T}$ & Solenosphaera omnitubus & 2 in 7 & 28 & 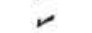 & & & & $\checkmark(575)$ \\
\hline B & Didymocyrtis avita & 1 in 7 & 14 & & & . & $r$ & $\sim(574 \mathrm{~A})$ \\
\hline$T$ & Spongaster berminghami & 2 in 7 & 28 & $\sim$ & $r$ & $r_{\text {r }}$ & $\sim$ & \\
\hline B & Pterocanium prismatium & 1 in 7 & 14 & & & $r^{b}$ & & \\
\hline$T$ & Didymocyrtis antepenultima & 1 in 7 & 14 & & & & & \\
\hline $\mathrm{T}$ & Stichocorys delmontensis & 1 in 7 & 14 & & r & & $r$ & \\
\hline $\mathrm{T}$ & Siphostichartus corona & 1 in 7 & 14 & $r$ & & & & $\checkmark(575)$ \\
\hline B & Solenosphaera omnitubus & 1 in 6 & 17 & & $r$ & & & \\
\hline $\mathrm{T}$ & Lithopera neotera & 1 in 6 & 17 & & $r$ & & r & \\
\hline B & Spongaster berminghami & 1 in 7 & 14 & $r$ & & 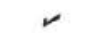 & & \\
\hline $\mathrm{T}$ & Stichocorys wolffii & 1 in 6 & 17 & 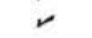 & & & $r$ & \\
\hline B & Lithopera bacca & 1 in 6 & 17 & & r & & 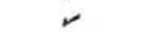 & \\
\hline $\mathrm{T}$ & Lithopera renzae & 1 in 6 & 17 & & $?$ & & 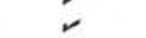 & $\sim(575 B)^{c}$ \\
\hline$T$ & Didymocyrtis mammifera & 1 in 6 & 17 & & ? & & & $-(575 B)^{c}$ \\
\hline B & Lithopera thornburgi & 1 in 6 & 17 & & ? & $r$ & & $-(575 B)^{c}$ \\
\hline $\mathrm{T}$ & Calocycletta virginis & 1 in 6 & 17 & $r$ & & & $\sim^{d}$ & \\
\hline $\mathrm{T}$ & Carpocanopsis bramlettei & 1 in 6 & 17 & $r$ & & & & \\
\hline$T$ & Dorcadospyris dentata & 1 in 5 & 20 & $r$ & & & & \\
\hline$T$ & Eucyrtidium diaphanes & 1 in 5 & 20 & $\checkmark$ & & & & \\
\hline $\mathrm{T}$ & Didymocyrtis prismatica & 1 in 5 & 20 & & $r$ & & & \\
\hline $\mathrm{T}$ & Carpocanopsis favosa & 1 in 6 & 17 & & $r$ & & & \\
\hline B & Lithopera renzae & 1 in 5 & 20 & $\checkmark$ & & & & \\
\hline $\mathrm{T}$ & Spirocyrtis subtilis & 2 in 6 & 30 & $r$ & $r$ & & & \\
\hline $\mathrm{T}$ & Dorcadospyris alata & 2 in 6 & 30 & & $\gamma^{e}$ & & & $\sim(575 B)^{c}$ \\
\hline B & Cyrtocapsella japonica & 2 in 6 & 30 & $r$ & & & & \\
\hline$T$ & Lychnocanoma elongata & 1 in 3 & $30^{f}$ & $r$ & & & & \\
\hline$T$ & Lychnodictyum audax & 3 in 8 & 37 & $r$ & $r$ & & & $\sim(574 A)$ \\
\hline $\mathrm{T}$ & Botryostrobus bramlettei & 3 in 7 & 43 & $r$ & $r$ & & ? & $\sim(574 \mathrm{~A}, 575)$ \\
\hline$T$ & Didymocyrtis laticonus & 3 in 7 & 43 & $r$ & $r$ & & r & \\
\hline $\mathrm{B}$ & Lithopera neotera & 4 in 6 & 67 & 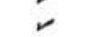 & $r$ & & & $\sim(575 \mathrm{~B})^{\mathrm{c}}$ \\
\hline $\mathrm{B}$ & Phormostichoartus corbula & 4 in 6 & 67 & r & 2 & & & $-(575 B)^{c}$ \\
\hline $\mathrm{B}$ & Dictyocoryne ontongensis & 3 in 6 & 50 & & r & $r$ & & $\sim(575 B)^{c}$ \\
\hline B & Carpocanopsis cristata s.s. & 2 in 5 & 40 & 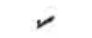 & & & & \\
\hline B & Didymocyrtis prismatica & 1 in 2 & $50^{\mathrm{f}}$ & $r$ & & & & \\
\hline
\end{tabular}

Note: Rules are used to separate events that are out sequence in $<30 \%, 30 \%$, and $>30 \%$ of the holes in which the event occurs (see text).

${ }^{\mathrm{a}} \mathrm{T}=$ top; $\mathrm{B}=$ bottom

$\mathrm{b}$ At lower end of range.

c A large section of Hole 575B shows serious mixing of radiolarian events, although no disturbance is indicated on the barrel sheets.

d Requires complete specimen for identification.

e Out of sequence by about $100 \mathrm{~m}$ in Hole 572D.

$f$ Event occurs in 3 or fewer holes.

Cyclampterium(?) milowi Sanfilippo and Riedel; Sanfilippo and Riedel, 1970

Dictyocoryne ontongensis Riedel and Sanfilippo; Riedel and Sanfilippo, 1971. Note: The lower limit of this species is difficult to place because specimens are so rare.

Didymocyrtis avita (Riedel); Riedel, 1953

Dorcadospyris alata (Riedel); Brachiospyris alata in Riedel, 1959; Riedel and Sanfilippo, 1970. Note: The upper limit of this species is seriously misplaced in Hole 572D.

Giraffospyris toxaria (Haeckel); Goll, 1969

Liriospyris parkerae Riedel and Sanfilippo; Riedel and Sanfilippo, 1971

Lithocyclia angusta (Riedel); Trigonactura angusta in Riedel, 1959; Riedel and Sanfilippo, 1970

Lithocyclia crux Moore; Moore, 1971

Lithopera bacca Ehrenberg; Nigrini, 1967

Lithopera neotera Sanfilippo and Riedel; Sanfilippo and Riedel, 1970. Note: The lower limit of this species appears to be an unreliable datum.
Lithopera renzae Sanfilippo and Riedel; Sanfilippo and Riedel, 1970 Theocyrtis tuberosa Riedel; Riedel 1959, and Riedel and Sanfilippo, 1971

Tristylospyris triceros (Ehrenberg); Haeckel, 1887

\section{REFERENCES}

Goll, R. M., 1969. Classification and phylogeny of Cenozoic Trissocyclidae (Radiolaria) in Pacific and Caribbean basins. Part II. J. Paleontol., 43(2):322-339.

, 1972. Radiolaria. In Hayes, J. D., et al., Init. Repts. DSDP, 9: Washington (U.S. Govt. Printing Office), 947-1058.

Haeckel, E., 1887. Report on the Radiolaria collected by H.M.S. Challenger during the years 1873-1876. Report Scientific Results, Voyage H.M.S. Challenger, Zoology (Vol. 18, 2 pts.).

Hardenbol, J., and Berggren, W. A., 1978. A new Paleogene numerical time scale. Stud. Geol., 6:213-214.

Knoll, A., and Johnson, D., 1975. Late Pleistocene evolution of the collosphaerid radiolarian Buccinosphaera invaginata Haeckel. Micropaleontology, 21:60-68. 


\section{A. NIGRINI}

Moore, T. C., 1971. Radiolaria. In Tracey, J. I., Jr., Sutton, G. H., et al., Init. Repts. DSDP, 8: Washington (U.S. Govt. Printing Office), 727-775.

Nigrini, C., 1967. Radiolaria in pelagic sediments from the Indian and Atlantic oceans. Bull. Scripps Inst. Oceanogr., 11:1-125.

, 1971. Radiolarian zones in the Quaternary of the equatorial Pacific Ocean. In Funnell, B. M., and Riedel, W. R. (Eds.), Micropaleontology of Oceans: Cambridge (Cambridge Univ. Press), pp. 443-461.

, 1977. Tropical Cenozoic Artostrobiidae (Radiolaria). Micropaleontology, 23(3):241-269.

Nigrini, C., and Lombari, G., 1984. A guide to Miocene Radiolaria. Cushman Found. Spec. Publ., 22:1-308.

Nigrini, C., and Moore, T. C., 1979. A guide to modern Radiolaria. Cushman Found. Spec. Publ., 16:1-260.

Riedel, W. R., 1953. Mesozoic and late Tertiary Radiolaria of Rotti. $J$. Paleontol., 27(6):805-813.

1959. Oligocene and lower Miocene Radiolaria in tropica Pacific sediments. Micropaleontology, 5(3):285-302.

Riedel, W. R., and Sanfilippo, A., 1970. Radiolaria, Leg 4, Deep Sea Drilling Project. In Bader, R. G., Gerard, R. D., et al., Init. Repts. DSDP, 4: Washington (U.S. Govt. Printing Office), 503-575.
1971. Cenozoic Radiolaria from the western tropical Pacific, Leg 7. In Winterer, E. L., Riedel, W. R., et al., Init. Repts. DSDP, 7, Pt. 2: Washington (U.S. Govt. Printing Office), 1529-1672.

1978. Stratigraphy and evolution of tropical Cenozoic radiolarians. Micropaleontology, 24(1):61-96.

Sanfilippo, A., and Riedel, W. R., 1970. Post-Eocene "closed" theoperid radiolarians. Micropaleontology, 16(4):446-462.

Sanfilippo, A., Westberg-Smith, M. J., and Riedel, W. R., in press. Cenozoic Radiolaria. In Bolli, H. M., Saunders, J. B., and PerchNielsen, K. (Eds.), Biostratigraphy by Marine Plankton (provisional title): Cambridge (Cambridge Univ. Press).

Westberg, M. J., and Riedel, W. R., 1982. Radiolaria from the Middle America Trench off Guatemala, Deep Sea Drilling Project Leg 67. In Aubouin, J., von Huene, R., et al., Init. Repts. DSDP, 67: Washington (U.S. Govt. Printing Office), 401-424.

Date of Initial Receipt: 3 November 1983 Date of Acceptance: 8 March 1984

APPENDIX A

Radiolarian Events, Sites 572 to 575

Table 1. List of radiolarian events for Site $572 .^{\mathrm{a}}$

\begin{tabular}{|c|c|c|c|c|c|c|c|c|c|}
\hline \multirow[b]{2}{*}{ Zone } & \multirow[b]{2}{*}{$\begin{array}{l}\text { Age } \\
(\mathrm{Ma})\end{array}$} & \multirow[b]{2}{*}{$\begin{array}{c}\text { Top } \\
\text { or } \\
\text { bottom }\end{array}$} & \multirow[b]{2}{*}{ Species } & \multicolumn{2}{|c|}{ Hole $572 \mathrm{~A}$} & \multicolumn{2}{|c|}{ Hole $572 \mathrm{C}$} & \multicolumn{2}{|c|}{ Hole 572D } \\
\hline & & & & Core-Section & $\begin{array}{l}\text { Sub-bottom } \\
\text { depth } \\
\text { (m) }\end{array}$ & Core-Section & $\begin{array}{l}\text { Sub-bottom } \\
\text { depth } \\
\text { (m) }\end{array}$ & Core-Section & $\begin{array}{l}\text { Sub-bottom } \\
\text { depth } \\
\text { (m) }\end{array}$ \\
\hline B. invaginata & 0.3 & B & $\begin{array}{c}\text { Buccinosphaera } \\
\text { invaginata }\end{array}$ & & & & & & \\
\hline \multirow[t]{3}{*}{ C. tuberosa } & 0.44 & $\mathrm{~T}$ & $\begin{array}{l}\text { Stylatractus } \\
\text { universus }\end{array}$ & $\begin{array}{l}1-3 \\
1, \mathrm{CC}\end{array}$ & $\begin{array}{l}3.70 \\
9.57\end{array}$ & $1, \mathrm{CC}$ & 9.50 & & \\
\hline & & B & $\begin{array}{c}\text { Collosphaera } \\
\text { tuberosa }\end{array}$ & $\begin{array}{l}1, \mathrm{CC} \\
2-3\end{array}$ & $\begin{array}{r}9.57 \\
13.20 \\
\end{array}$ & $\begin{array}{l}1, \mathrm{CC} \\
2, \mathrm{CC}\end{array}$ & $\begin{array}{r}9.50 \\
19.67 \\
\end{array}$ & & \\
\hline & & B & $\begin{array}{l}\text { Pterocorys } \\
\text { hertwigii }\end{array}$ & $\begin{array}{l}2-3 \\
2, \mathrm{CC}\end{array}$ & $\begin{array}{l}13.20 \\
18.59 \\
\end{array}$ & $\begin{array}{l}1, \mathrm{CC} \\
2, \mathrm{CC}\end{array}$ & $\begin{array}{r}9.50 \\
19.67 \\
\end{array}$ & & \\
\hline A. ypsilon & 1.1 & $\mathrm{~T}$ & $\begin{array}{c}\text { Anthocyrtidium } \\
\text { angulare }\end{array}$ & $\begin{array}{l}2-5 \\
2, \mathrm{CC}\end{array}$ & $\begin{array}{l}16.20 \\
18.59\end{array}$ & $\begin{array}{l}1, \mathrm{CC} \\
2, \mathrm{CC}\end{array}$ & $\begin{array}{r}9.50 \\
19.67 \\
\end{array}$ & & \\
\hline \multirow[t]{4}{*}{ A. angulare } & & $\mathbf{T}$ & $\begin{array}{c}\text { Theocorythium } \\
\text { vetulum }\end{array}$ & $\begin{array}{l}2-5 \\
2, \mathrm{CC} \\
\end{array}$ & $\begin{array}{l}16.20 \\
18.59 \\
\end{array}$ & $\begin{array}{l}2, \mathrm{CC} \\
3, \mathrm{CC} \\
\end{array}$ & $\begin{array}{l}19.67 \\
29.33 \\
\end{array}$ & 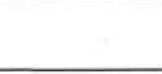 & \\
\hline & & $\mathbf{T}$ & $\begin{array}{l}\text { Didymocyrtis } \\
\text { avita }\end{array}$ & $\begin{array}{l}2-5 \\
2, \mathrm{CC}\end{array}$ & $\begin{array}{l}16.20 \\
18.59 \\
\end{array}$ & $\begin{array}{l}2, \mathrm{CC} \\
3, \mathrm{CC}\end{array}$ & $\begin{array}{l}19.67 \\
29.33 \\
\end{array}$ & & \\
\hline & & B & $\begin{array}{c}\text { Lamprocyrtis } \\
\text { nigriniae }\end{array}$ & $\begin{array}{l}2, \mathrm{CC} \\
3-3\end{array}$ & $\begin{array}{l}18.59 \\
22.91 \\
\end{array}$ & $\begin{array}{l}2, \mathrm{CC} \\
3, \mathrm{CC} \\
\end{array}$ & $\begin{array}{l}19.67 \\
29.33 \\
\end{array}$ & & \\
\hline & 1.35 & B & $\begin{array}{c}\text { Theocorythium } \\
\text { trachelium }\end{array}$ & $\begin{array}{l}2, \mathrm{CC} \\
3-3\end{array}$ & $\begin{array}{l}18.59 \\
22.91\end{array}$ & $\begin{array}{l}2, \mathrm{CC} \\
3, \mathrm{CC}\end{array}$ & $\begin{array}{l}19.67 \\
29.33 \\
\end{array}$ & & \\
\hline \multirow[t]{2}{*}{ P. prismatium } & 1.5 & $\mathrm{~T}$ & $\begin{array}{l}\text { Pterocanium } \\
\text { prismatium }\end{array}$ & $\begin{array}{l}3-3 \\
3, \mathrm{CC} \\
\end{array}$ & $\begin{array}{l}22.91 \\
28.56 \\
\end{array}$ & $\begin{array}{l}2, \mathrm{CC} \\
3, \mathrm{CC} \\
\end{array}$ & $\begin{array}{l}19.67 \\
29.33 \\
\end{array}$ & & . \\
\hline & & B & $\begin{array}{c}\text { Anthocyrtidium } \\
\text { angulare }\end{array}$ & $\begin{array}{l}3-5 \\
3, \mathrm{CC}\end{array}$ & $\begin{array}{l}25.91 \\
28.56\end{array}$ & $\begin{array}{l}2, \mathrm{CC} \\
3, \mathrm{CC} \\
\end{array}$ & $\begin{array}{l}19.67 \\
29.33 \\
\end{array}$ & & \\
\hline \multirow[t]{6}{*}{ S. pentas } & 2.4 & $\mathrm{~T}$ & $\begin{array}{r}\text { Stichocorys } \\
\text { peregrina }\end{array}$ & $\begin{array}{l}5-3 \\
5, \mathrm{CC} \\
\end{array}$ & $\begin{array}{l}40.01 \\
45.84 \\
\end{array}$ & $\begin{array}{l}4, \mathrm{CC} \\
5, \mathrm{CC} \\
\end{array}$ & $\begin{array}{l}38.77 \\
48.44 \\
\end{array}$ & & \\
\hline & & $\mathbf{T}$ & $\begin{array}{l}\text { Phormostichoartus } \\
\text { fistula }\end{array}$ & $\begin{array}{l}5, \mathrm{CC} \\
6-3 \\
\end{array}$ & $\begin{array}{l}45.84 \\
49.61 \\
\end{array}$ & $\begin{array}{l}4, \mathrm{CC} \\
5, \mathrm{CC} \\
\end{array}$ & $\begin{array}{l}38.77 \\
48.44 \\
\end{array}$ & & \\
\hline & 3.4 & $\mathbf{T}$ & $\begin{array}{c}\text { Spongaster } \\
\text { pentas }\end{array}$ & $\begin{array}{l}6-3 \\
6, \mathrm{CC} \\
\end{array}$ & $\begin{array}{l}49.61 \\
55.13 \\
\end{array}$ & $\begin{array}{l}5, \mathrm{CC} \\
6, \mathrm{CC} \\
\end{array}$ & $\begin{array}{l}48.44 \\
58.07 \\
\end{array}$ & & \\
\hline & & $\mathbf{T}$ & $\begin{array}{l}\text { Lychnodictyum } \\
\text { audax }\end{array}$ & $\begin{array}{l}6-3 \\
6, \mathrm{CC} \\
\end{array}$ & $\begin{array}{l}49.61 \\
55.13 \\
\end{array}$ & $\begin{array}{l}5, \mathrm{CC} \\
6, \mathrm{CC} \\
\end{array}$ & $\begin{array}{l}48.44 \\
58.07 \\
\end{array}$ & & \\
\hline & & $\mathbf{T}$ & $\begin{array}{l}\text { Phormostichoartus } \\
\text { doliolum }\end{array}$ & $\begin{array}{l}6, \mathrm{CC} \\
7-3 \\
\end{array}$ & $\begin{array}{l}55.13 \\
58.91 \\
\end{array}$ & $\begin{array}{l}5, C C \\
6, C C \\
\end{array}$ & $\begin{array}{l}48.44 \\
58.07\end{array}$ & & \\
\hline & & B & $\begin{array}{l}\text { Amphirhopalum } \\
\text { ypsilon }\end{array}$ & $\begin{array}{l}6, \mathrm{CC} \\
7-3\end{array}$ & $\begin{array}{l}55.13 \\
58.91\end{array}$ & $\begin{array}{l}6, \mathrm{CC} \\
7, \mathrm{CC}\end{array}$ & $\begin{array}{l}58.07 \\
67.75\end{array}$ & & \\
\hline
\end{tabular}

a Pairs of levels (sections and the corresponding depths in meters below the sediment surface) bracket the level at which an event occurs. Events and absolute ages in brackets are inconsistent with the majority of data collected. 
Table 1. (Continued).

\begin{tabular}{|c|c|c|c|c|c|c|c|c|c|}
\hline \multirow[b]{2}{*}{ Zone } & \multirow[b]{2}{*}{$\begin{array}{l}\text { Age } \\
\text { (Ma) }\end{array}$} & \multirow[b]{2}{*}{$\begin{array}{c}\text { Top } \\
\text { or } \\
\text { bottom }\end{array}$} & \multirow[b]{2}{*}{ Species } & \multicolumn{2}{|c|}{ Hole $572 \mathrm{~A}$} & \multicolumn{2}{|c|}{ Hole $572 \mathrm{C}$} & \multicolumn{2}{|c|}{ Hole 572D } \\
\hline & & & & Core-Section & $\begin{array}{l}\text { Sub-bottom } \\
\text { depth } \\
\text { (m) }\end{array}$ & Core-Section & $\begin{array}{l}\text { Sub-bottom } \\
\text { depth } \\
\text { (m) }\end{array}$ & Core-Section & $\begin{array}{l}\text { Sub-bottom } \\
\text { depth } \\
\text { (m) }\end{array}$ \\
\hline \multirow[t]{7}{*}{ S. pentas } & & B & $\begin{array}{l}\text { Didymocyrtis } \\
\quad \text { tetrathalamus }\end{array}$ & $\begin{array}{l}6, \mathrm{CC} \\
7, \mathrm{CC}\end{array}$ & $\begin{array}{l}55.13 \\
63.99\end{array}$ & $\begin{array}{l}6, \mathrm{CC} \\
7, \mathrm{CC}\end{array}$ & $\begin{array}{l}58.07 \\
63.99\end{array}$ & & \\
\hline & 3.6 & B & $\begin{array}{c}\text { Spongaster } \\
\text { tetras }\end{array}$ & $\begin{array}{l}7-3 \\
7, \mathrm{CC}\end{array}$ & $\begin{array}{l}58.91 \\
63.99\end{array}$ & $\begin{array}{l}6, \mathrm{CC} \\
7, \mathrm{CC}\end{array}$ & $\begin{array}{l}58.07 \\
67.75\end{array}$ & & \\
\hline & 3.69 & $\mathrm{~T}$ & $\begin{array}{r}\text { Didymocyrtis } \\
\text { penultima }\end{array}$ & $\begin{array}{l}7-3 \\
7, \mathrm{CC}\end{array}$ & $\begin{array}{l}58.91 \\
63.99\end{array}$ & $\begin{array}{l}\text { 6,CC } \\
7, \mathrm{CC}\end{array}$ & $\begin{array}{l}58.07 \\
67.75\end{array}$ & & \\
\hline & & $\mathrm{T}$ & $\begin{array}{r}\text { Solenosphaera } \\
\text { omnitubus }\end{array}$ & $\begin{array}{l}7-3 \\
7, \mathrm{CC}\end{array}$ & $\begin{array}{l}58.91 \\
63.99\end{array}$ & {$\left[\begin{array}{l}5, \mathrm{CC} \\
6, \mathrm{CC}\end{array}\right.$} & $\left.\begin{array}{l}48.44 \\
58.07\end{array}\right]$ & & \\
\hline & & B & $\begin{array}{c}\text { Didymocyrtis } \\
\text { avita }\end{array}$ & $\begin{array}{l}7, \mathrm{CC} \\
8-3\end{array}$ & $\begin{array}{l}63.99 \\
67.81\end{array}$ & $\begin{array}{l}8, \mathrm{CC} \\
9, \mathrm{CC}\end{array}$ & $\begin{array}{l}77.24 \\
86.62\end{array}$ & & \\
\hline & & $\mathrm{T}$ & $\begin{array}{l}\text { Spongaster } \\
\quad \text { berminghami }\end{array}$ & $\begin{array}{l}9-3 \\
9, \mathrm{CC}\end{array}$ & $\begin{array}{l}76.01 \\
81.71\end{array}$ & {$\left[\begin{array}{l}10, \mathrm{CC} \\
11, \mathrm{CC}\end{array}\right.$} & $\left.\begin{array}{r}94.46 \\
101.01\end{array}\right]$ & & \\
\hline & & & $\begin{array}{l}S . \text { berminghami } \\
\rightarrow S \text {. pentas }\end{array}$ & $\begin{array}{l}9-3 \\
9, \mathrm{CC}\end{array}$ & $\begin{array}{l}76.01 \\
81.71\end{array}$ & $\begin{array}{l}8, \mathrm{CC} \\
11, \mathrm{CC}\end{array}$ & $\begin{array}{r}77.24 \\
101.01 \\
\end{array}$ & & \\
\hline \multirow[t]{10}{*}{ S. peregrina } & 4.3 & B & $\begin{array}{c}\text { Spongaster } \\
\text { pentas }\end{array}$ & $\begin{array}{l}9-3 \\
9, \mathrm{CC}\end{array}$ & $\begin{array}{l}76.01 \\
81.71\end{array}$ & $\begin{array}{l}8, \mathrm{CC} \\
9, \mathrm{CC}\end{array}$ & $\begin{array}{l}77.24 \\
86.62\end{array}$ & & \\
\hline & & $T$ & $\begin{array}{r}\text { Botryostrobus } \\
\text { bramlettei } \\
\end{array}$ & $\begin{array}{l}10, \mathrm{CC} \\
11-3\end{array}$ & $\begin{array}{l}91.38 \\
95.19\end{array}$ & $\begin{array}{l}8, \mathrm{CC} \\
9, \mathrm{CC}\end{array}$ & $\begin{array}{l}77.24 \\
86.62\end{array}$ & & \\
\hline & 4.8 & B & $\begin{array}{l}\text { Pterocanium } \\
\quad \text { prismatium }\end{array}$ & $\begin{array}{l}11, \mathrm{CC} \\
12-3\end{array}$ & $\begin{array}{l}100.63 \\
104.39\end{array}$ & $\begin{array}{l}10, \mathrm{CC} \\
11, \mathrm{CC}\end{array}$ & $\begin{array}{r}96.46 \\
101.01\end{array}$ & & \\
\hline & & B & $\begin{array}{l}\text { Botryostrobus } \\
\text { aquilonaris }\end{array}$ & $\begin{array}{l}13-3 \\
13, \mathrm{CC}\end{array}$ & $\begin{array}{l}113.61 \\
119.08\end{array}$ & $\begin{array}{l}12, \mathrm{CC} \\
13, \mathrm{CC}\end{array}$ & $\begin{array}{l}106.39 \\
111.46\end{array}$ & & \\
\hline & & $\mathrm{T}$ & $\begin{array}{l}\text { Didymocyrtis } \\
\quad \text { antepenultima }\end{array}$ & $\begin{array}{l}13-3 \\
13, \mathrm{CC}\end{array}$ & $\begin{array}{l}113.61 \\
119.08\end{array}$ & $\begin{array}{l}12, \mathrm{CC} \\
13, \mathrm{CC}\end{array}$ & $\begin{array}{l}106.39 \\
111.46\end{array}$ & & \\
\hline & & $\mathrm{T}$ & $\begin{array}{l}\text { Stichocorys } \\
\quad \text { delmontensis }\end{array}$ & $\begin{array}{l}13-3 \\
13, \mathrm{CC}\end{array}$ & $\begin{array}{l}113.61 \\
119.08\end{array}$ & $\begin{array}{l}12, \mathrm{CC} \\
13, \mathrm{CC}\end{array}$ & $\begin{array}{l}106.39 \\
111.46\end{array}$ & & \\
\hline & & $\mathrm{T}$ & $\begin{array}{l}\text { Siphostichartus } \\
\text { corona }\end{array}$ & $\begin{array}{l}13-3 \\
13, \mathrm{CC}\end{array}$ & $\begin{array}{l}113.61 \\
119.08\end{array}$ & $\begin{array}{l}13, \mathrm{CC} \\
14, \mathrm{CC}\end{array}$ & $\begin{array}{l}111.46 \\
119.34\end{array}$ & & \\
\hline & 5.0 & $T$ & $\begin{array}{c}\text { Acrobotrys } \\
\text { tritubus }\end{array}$ & $\begin{array}{l}14, C C \\
15-3\end{array}$ & $\begin{array}{l}128.23 \\
132.01\end{array}$ & $\begin{array}{l}15, \mathrm{CC} \\
16, \mathrm{CC}\end{array}$ & $\begin{array}{l}130.23 \\
139.99\end{array}$ & & \\
\hline & & $\mathrm{T}$ & $\begin{array}{c}\text { Calocycletta } \\
\text { caepa }\end{array}$ & $\begin{array}{l}16-3 \\
16, \mathrm{CC}\end{array}$ & $\begin{array}{l}140.82 \\
145.20\end{array}$ & $\begin{array}{l}15, \mathrm{CC} \\
16, \mathrm{CC}\end{array}$ & $\begin{array}{l}130.23 \\
139.99\end{array}$ & $\begin{array}{l}2-3 \\
2, \mathrm{CC}\end{array}$ & $\begin{array}{l}164.15 \\
170.24\end{array}$ \\
\hline & & & $\begin{array}{l}\text { S. delmontensis } \\
\rightarrow S \text {. peregrina }\end{array}$ & & & & & $\begin{array}{l}2, \mathrm{CC} \\
3, \mathrm{CC}\end{array}$ & $\begin{array}{l}170.24 \\
179.71\end{array}$ \\
\hline \multirow[t]{3}{*}{ D. penultima } & & $\mathrm{T}$ & $\begin{array}{c}\text { Phormostichoartus } \\
\text { marylandicus }\end{array}$ & & & & & $\begin{array}{l}6, \mathrm{CC} \\
7-3\end{array}$ & $\begin{array}{l}208.07 \\
211.61\end{array}$ \\
\hline & 6.55 & B & $\begin{array}{r}\text { Solenosphaera } \\
\text { omnitubus }\end{array}$ & & & & & {$\left[\begin{array}{l}4, \mathrm{CC} \\
5-4\end{array}\right.$} & $\left.\begin{array}{l}189.02 \\
194.15\end{array}\right]$ \\
\hline & {$[6.4]$} & B & $\begin{array}{c}\text { Stichocorys } \\
\text { peregrina }\end{array}$ & & & & & $\begin{array}{l}7-3 \\
7, \mathrm{CC}\end{array}$ & $\begin{array}{l}211.61 \\
217.62 \\
\end{array}$ \\
\hline \multirow[t]{8}{*}{ D. antepenultima } & 8.2 & $\mathrm{~T}$ & $\begin{array}{l}\text { Diartus } \\
\quad \text { hughesi }\end{array}$ & & & & & $\begin{array}{l}7, \mathrm{CC} \\
8-3\end{array}$ & $\begin{array}{l}217.62 \\
221.11\end{array}$ \\
\hline & & $\mathrm{T}$ & $\begin{array}{l}\text { Lithopera } \\
\text { neotera }\end{array}$ & & & & & {$\left[\begin{array}{l}6-5 \\
6, \mathrm{CC}\end{array}\right.$} & $\left.\begin{array}{l}205.11 \\
208.07\end{array}\right]$ \\
\hline & & $\mathrm{T}$ & $\begin{array}{l}\text { Dictyocoryne } \\
\quad \text { ontongensis }\end{array}$ & & & & & $\begin{array}{l}8, \mathrm{CC} \\
9, \mathrm{CC} \\
\end{array}$ & $\begin{array}{l}227.23 \\
231.97 \\
\end{array}$ \\
\hline & & $\mathrm{T}$ & $\begin{array}{l}\text { Botryostrobus } \\
\quad \text { miralestensis }\end{array}$ & & & & & $\begin{array}{l}8, \mathrm{CC} \\
9, \mathrm{CC} \\
\end{array}$ & $\begin{array}{l}227.23 \\
231.97 \\
\end{array}$ \\
\hline & {$[11.2]$} & B & $\begin{array}{c}\text { Acrobotrys } \\
\text { tritubus }\end{array}$ & & & & & $\begin{array}{l}9, \mathrm{CC} \\
10-3\end{array}$ & $\begin{array}{l}231.97 \\
240.11\end{array}$ \\
\hline & & $\mathrm{T}$ & $\begin{array}{c}\text { Didymocyrtis } \\
\text { laticonus }\end{array}$ & & & & & $\begin{array}{l}10-3 \\
10, \mathrm{CC}\end{array}$ & $\begin{array}{l}240.11 \\
246.10 \\
\end{array}$ \\
\hline & 8.2 & B & $\begin{array}{r}\text { Didymocyrtis } \\
\text { penultima } \\
\end{array}$ & & & & & $\begin{array}{l}10-3 \\
10, \mathrm{CC}\end{array}$ & $\begin{array}{l}240.11 \\
246.10\end{array}$ \\
\hline & 9.7 & $\mathrm{~T}$ & $\begin{array}{l}\text { Diartus } \\
\quad \text { petterssoni }\end{array}$ & & & & & $\begin{array}{l}10, \mathrm{CC} \\
11-3\end{array}$ & $\begin{array}{l}246.10 \\
249.61\end{array}$ \\
\hline
\end{tabular}


Table 1. (Continued).

\begin{tabular}{|c|c|c|c|c|c|c|c|c|c|}
\hline \multirow[b]{2}{*}{ Zone } & \multirow[b]{2}{*}{$\begin{array}{l}\text { Age } \\
(\mathrm{Ma})\end{array}$} & \multirow[b]{2}{*}{$\begin{array}{c}\text { Top } \\
\text { or } \\
\text { bottom }\end{array}$} & \multirow[b]{2}{*}{ Species } & \multicolumn{2}{|c|}{ Hole $572 \mathrm{~A}$} & \multicolumn{2}{|c|}{ Hole $572 \mathrm{C}$} & \multicolumn{2}{|c|}{ Hole 572D } \\
\hline & & & & Core-Section & $\begin{array}{l}\text { Sub-bottom } \\
\text { depth } \\
\text { (m) }\end{array}$ & Core-Section & $\begin{array}{l}\text { Sub-bottom } \\
\text { depth } \\
\text { (m) }\end{array}$ & Core-Section & $\begin{array}{l}\text { Sub-bottom } \\
\text { depth } \\
\text { (m) }\end{array}$ \\
\hline \multirow[t]{2}{*}{ D. antepenultima } & & B & $\begin{array}{l}\text { Spongaster } \\
\text { berminghami }\end{array}$ & & & & & $\begin{array}{l}11-3 \\
11, \mathrm{CC}\end{array}$ & $\begin{array}{l}249.61 \\
255.68\end{array}$ \\
\hline & & & $\begin{array}{l}\text { D. petterssoni } \\
\rightarrow \text { D. hughesi }\end{array}$ & & & & & $\begin{array}{l}11-3 \\
13-3\end{array}$ & $\begin{array}{l}249.61 \\
268.61\end{array}$ \\
\hline \multirow[t]{18}{*}{ D. petterssoni } & 11.5 & B & $\begin{array}{l}\text { Diartus } \\
\quad \text { hughesi }\end{array}$ & & & & & $\begin{array}{l}13-3 \\
13, \mathrm{CC}\end{array}$ & $\begin{array}{l}268.61 \\
272.45\end{array}$ \\
\hline & 11.6 & $T$ & $\begin{array}{c}\text { Stichocorys } \\
\text { wolffii }\end{array}$ & & & & & {$\left[\begin{array}{l}12, \mathrm{CC} \\
13-3\end{array}\right.$} & $\left.\begin{array}{l}261.79 \\
268.61\end{array}\right]$ \\
\hline & & $\mathrm{T}$ & $\begin{array}{c}\text { Cyrtocapsella } \\
\text { japonica }\end{array}$ & & & & & $\begin{array}{l}14, \mathrm{CC} \\
15, \mathrm{CC}\end{array}$ & $\begin{array}{l}281.87 \\
287.83\end{array}$ \\
\hline & & B & $\begin{array}{l}\text { Phormostichoartus } \\
\text { doliolum }\end{array}$ & & & & & $\begin{array}{l}14, \mathrm{CC} \\
15, \mathrm{CC}\end{array}$ & $\begin{array}{l}281.87 \\
287.83\end{array}$ \\
\hline & & $\mathrm{T}$ & $\begin{array}{l}\text { Lithopera } \\
\quad \text { thornburgi }\end{array}$ & & & & & $\begin{array}{l}14, \mathrm{CC} \\
15, \mathrm{CC}\end{array}$ & $\begin{array}{l}281.83 \\
287.83\end{array}$ \\
\hline & & $\mathbf{T}$ & $\begin{array}{r}\text { Spirocyrtis } \\
\text { subtilis }\end{array}$ & & & & & $\begin{array}{l}14, \mathrm{CC} \\
15, \mathrm{CC}\end{array}$ & $\begin{array}{l}281.83 \\
287.83\end{array}$ \\
\hline & & B & $\begin{array}{l}\text { Didymocyrtis } \\
\text { antepenultima }\end{array}$ & & & & & $\begin{array}{l}15, \mathrm{CC} \\
16-3\end{array}$ & $\begin{array}{l}287.83 \\
297.11\end{array}$ \\
\hline & & B & $\begin{array}{r}\text { Lithopera } \\
\text { bacca }\end{array}$ & & & & & {$\left[\begin{array}{l}14-3 \\
14, C C\end{array}\right.$} & $\left.\begin{array}{l}278.11 \\
281.83\end{array}\right]$ \\
\hline & & $\mathrm{T}$ & $\begin{array}{r}\text { Carpocanopsis } \\
\text { cristata } \text { s.s. }\end{array}$ & & & & & $\begin{array}{l}16-3 \\
16, \mathrm{CC}\end{array}$ & $\begin{array}{l}297.11 \\
302.93 \\
\end{array}$ \\
\hline & & B & $\begin{array}{r}\text { Botryostrobus } \\
\text { bramlettei }\end{array}$ & & & & & $\begin{array}{l}20, \mathrm{CC} \\
21, \mathrm{CC}\end{array}$ & $\begin{array}{l}334.96 \\
348.54 \\
\end{array}$ \\
\hline & 11.8 & $\mathrm{~T}$ & $\begin{array}{c}\text { Cyrtocapsella } \\
\text { cornuta }\end{array}$ & & & & & $\begin{array}{l}20, \mathrm{CC} \\
21, \mathrm{CC}\end{array}$ & $\begin{array}{l}334.96 \\
348.54 \\
\end{array}$ \\
\hline & {$[12.5]$} & $\mathrm{T}$ & $\begin{array}{c}\text { Cyrtocapsella } \\
\text { tetrapera }\end{array}$ & & & & & $\begin{array}{l}20, \mathrm{CC} \\
21, \mathrm{CC}\end{array}$ & $\begin{array}{l}334.96 \\
348.54 \\
\end{array}$ \\
\hline & & $\mathrm{T}$ & $\begin{array}{r}\text { Lithopera } \\
\text { renzae }\end{array}$ & & & & & $\begin{array}{l}21, \mathrm{CC} \\
22, \mathrm{CC}\end{array}$ & $\begin{array}{l}348.54 \\
354.70\end{array}$ \\
\hline & 11.8 & $\mathrm{~T}$ & $\begin{array}{l}\text { Dorcadospyris } \\
\text { alata }\end{array}$ & & & & & {$\left[\begin{array}{l}31, \mathrm{CC} \\
32-3\end{array}\right.$} & $\left.\begin{array}{l}444.45 \\
458.61\end{array}\right]$ \\
\hline & & $\mathrm{T}$ & $\begin{array}{c}\text { Giraffospyris } \\
\text { toxaria }\end{array}$ & & & & & $\begin{array}{l}22, \mathrm{CC} \\
23-3\end{array}$ & $\begin{array}{l}354.70 \\
363.61\end{array}$ \\
\hline & & B & $\begin{array}{c}\text { Cyrtocapsella } \\
\text { japonica }\end{array}$ & & & & & $\begin{array}{l}23-3 \\
23, \mathrm{CC}\end{array}$ & $\begin{array}{l}363.61 \\
369.40 \\
\end{array}$ \\
\hline & & $\mathrm{T}$ & $\begin{array}{l}\text { Didymocyrtis } \\
\text { mammifera }\end{array}$ & & & & & $\begin{array}{l}23-3 \\
23, \mathrm{CC}\end{array}$ & $\begin{array}{l}363.61 \\
369.40 \\
\end{array}$ \\
\hline & 12.3 & B & $\begin{array}{l}\text { Diartus } \\
\quad \text { petterssoni }\end{array}$ & & & & & $\begin{array}{l}24-3 \\
24, \mathrm{CC} \\
\end{array}$ & $\begin{array}{l}373.11 \\
378.58 \\
\end{array}$ \\
\hline \multirow[t]{8}{*}{ D. alata } & 14.2 & B & $\begin{array}{l}\text { Lithopera } \\
\text { neotera }\end{array}$ & & & & & {$\left[\begin{array}{l}23, C \\
24-3\end{array}\right.$} & $\left.\begin{array}{l}369.40 \\
373.11\end{array}\right]$ \\
\hline & & B & $\begin{array}{l}\text { Lithopera } \\
\text { thornburgi }\end{array}$ & & & & & $\begin{array}{l}26, \mathrm{CC} \\
27-3\end{array}$ & $\begin{array}{l}397.03 \\
401.61 \\
\end{array}$ \\
\hline & & B & $\begin{array}{l}\text { Phormostichoartus } \\
\text { corbula }\end{array}$ & & & & & {$\left[\begin{array}{l}28, \mathrm{CC} \\
29, \mathrm{CC}\end{array}\right.$} & $\left.\begin{array}{l}414.68 \\
423.99\end{array}\right]$ \\
\hline & & B & $\begin{array}{l}\text { Dictyocoryne } \\
\text { ontongensis }\end{array}$ & & & & & {$\left[\begin{array}{l}25, \mathrm{CC} \\
26-3\end{array}\right.$} & $\left.\begin{array}{l}388.69 \\
392.11\end{array}\right]$ \\
\hline & {$[13.8]$} & B & $\begin{array}{c}\text { Didymocyrtis } \\
\text { laticonus }\end{array}$ & & & & 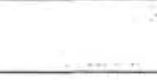 & $\begin{array}{l}27, \mathrm{CC} \\
28, \mathrm{CC}\end{array}$ & $\begin{array}{l}407.61 \\
414.68 \\
\end{array}$ \\
\hline & & $\mathrm{T}$ & $\begin{array}{l}\text { Liriospyris } \\
\text { parkerae }\end{array}$ & & & & $=4+n+6=$ & $\begin{array}{l}30, \mathrm{CC} \\
31-3\end{array}$ & $\begin{array}{l}433.83 \\
439.61\end{array}$ \\
\hline & 14.75 & $\mathrm{~T}$ & $\begin{array}{c}\text { Calocycletta } \\
\text { virginis }\end{array}$ & & & & & $\begin{array}{l}30, \mathrm{CC} \\
31-3 \\
\end{array}$ & $\begin{array}{l}433.83 \\
439.61\end{array}$ \\
\hline & & $\mathrm{T}$ & $\begin{array}{c}\text { Carpocanopsis } \\
\text { bramlettei }\end{array}$ & & & & & $\begin{array}{l}31-3 \\
31, \mathrm{CC}\end{array}$ & $\begin{array}{l}439.61 \\
444.45\end{array}$ \\
\hline
\end{tabular}


Table 1. (Continued).

\begin{tabular}{|c|c|c|c|c|c|c|c|c|c|}
\hline \multirow[b]{2}{*}{ Zone } & \multirow[b]{2}{*}{$\begin{array}{l}\text { Age } \\
\text { (Ma) }\end{array}$} & \multirow[b]{2}{*}{$\begin{array}{c}\text { Top } \\
\text { or } \\
\text { bottom }\end{array}$} & \multirow[b]{2}{*}{ Species } & \multicolumn{2}{|c|}{ Hole $572 \mathrm{~A}$} & \multicolumn{2}{|c|}{ Hole $572 \mathrm{C}$} & \multicolumn{2}{|c|}{ Hole 572D } \\
\hline & & & & Core-Section & $\begin{array}{l}\text { Sub-bottom } \\
\text { depth } \\
\text { (m) }\end{array}$ & Core-Section & $\begin{array}{l}\text { Sub-bottom } \\
\text { depth } \\
\text { (m) }\end{array}$ & Core-Section & $\begin{array}{l}\text { Sub-bottom } \\
\text { depth } \\
\text { (m) }\end{array}$ \\
\hline \multirow[t]{3}{*}{ D. alata } & & $\mathrm{T}$ & $\begin{array}{l}\text { Acrocubus } \\
\text { octopylus }\end{array}$ & & & & & $\begin{array}{l}31-3 \\
31, \mathrm{CC}\end{array}$ & $\begin{array}{l}439.61 \\
444.45\end{array}$ \\
\hline & 15.35 & $\mathrm{~T}$ & $\begin{array}{c}\text { Calocycletta } \\
\text { costata }\end{array}$ & & & & & $\begin{array}{l}31-3 \\
31, \mathrm{CC}\end{array}$ & $\begin{array}{l}439.61 \\
444.45\end{array}$ \\
\hline & & $\mathrm{T}$ & $\begin{array}{c}\text { Didymocyrtis } \\
\text { tubaria }\end{array}$ & & & & & $\begin{array}{l}32-4 \\
32, \mathrm{CC}\end{array}$ & $\begin{array}{l}450.61 \\
454.03\end{array}$ \\
\hline
\end{tabular}

Table 2. List of radiolarian events for Site 573.

\begin{tabular}{|c|c|c|c|c|c|c|c|c|c|}
\hline \multirow[b]{2}{*}{ Zone } & \multirow[b]{2}{*}{$\begin{array}{l}\text { Age } \\
(\mathrm{Ma})\end{array}$} & \multirow[b]{2}{*}{$\begin{array}{c}\text { Top } \\
\text { or } \\
\text { bottom }\end{array}$} & \multirow[b]{2}{*}{ Species } & \multicolumn{2}{|c|}{ Hole 573} & \multicolumn{2}{|c|}{ Hole 573A } & \multicolumn{2}{|c|}{ Hole 573B } \\
\hline & & & & Core-Section & $\begin{array}{l}\text { Sub-bottom } \\
\text { depth } \\
\text { (m) }\end{array}$ & Core-Section & $\begin{array}{l}\text { Sub-bottom } \\
\text { depth } \\
\text { (m) }\end{array}$ & Core-Section & $\begin{array}{l}\text { Sub-bottom } \\
\text { depth } \\
\text { (m) }\end{array}$ \\
\hline B. invaginata & 0.3 & B & $\begin{array}{c}\text { Buccinosphaera } \\
\text { invaginata }\end{array}$ & $\begin{array}{l}1-1 \\
1, \mathrm{CC}\end{array}$ & $\begin{array}{l}0.09 \\
1.97\end{array}$ & & & & \\
\hline \multirow[t]{3}{*}{ C. tuberosa } & 0.44 & $\mathrm{~T}$ & $\begin{array}{l}\text { Stylatractus } \\
\text { universus }\end{array}$ & $\begin{array}{l}1, \mathrm{CC} \\
2-3\end{array}$ & $\begin{array}{l}1.97 \\
6.31\end{array}$ & $1, \mathrm{CC}$ & $\begin{array}{r}0.00 \\
14.09\end{array}$ & & \\
\hline & & B & $\begin{array}{c}\text { Collosphaera } \\
\text { tuberosa }\end{array}$ & $\begin{array}{l}2-3 \\
2, \mathrm{CC}\end{array}$ & $\begin{array}{r}6.31 \\
11.46\end{array}$ & & & & \\
\hline & & B & $\begin{array}{l}\text { Pterocorys } \\
\text { hertwigii }\end{array}$ & $\begin{array}{l}2, \mathrm{CC} \\
3-3\end{array}$ & $\begin{array}{l}11.46 \\
15.81\end{array}$ & $\begin{array}{l}1, \mathrm{CC} \\
2, \mathrm{CC}\end{array}$ & $\begin{array}{l}14.09 \\
22.00\end{array}$ & & \\
\hline A. ypsilon & 1.1 & $\mathbf{T}$ & $\begin{array}{c}\text { Anthocyrtidium } \\
\text { angulare }\end{array}$ & $\begin{array}{l}3-3 \\
3-5 \\
\end{array}$ & $\begin{array}{l}15.81 \\
18.81 \\
\end{array}$ & $\begin{array}{l}1, \mathrm{CC} \\
2, \mathrm{CC}\end{array}$ & $\begin{array}{l}14.09 \\
22.00 \\
\end{array}$ & & \\
\hline \multirow[t]{4}{*}{ A. angulare } & & $T$ & $\begin{array}{c}\text { Theocorythium } \\
\text { vetulum }\end{array}$ & $\begin{array}{l}3-5 \\
3, \mathrm{CC} \\
\end{array}$ & $\begin{array}{l}18.81 \\
20.76 \\
\end{array}$ & $\begin{array}{l}1, \mathrm{CC} \\
2, \mathrm{CC}\end{array}$ & $\begin{array}{l}14.09 \\
22.00 \\
\end{array}$ & & \\
\hline & & $\mathrm{T}$ & $\begin{array}{l}\text { Didymocyrtis } \\
\text { avita }\end{array}$ & $\begin{array}{l}3-5 \\
4-3 \\
\end{array}$ & $\begin{array}{l}18.81 \\
25.31\end{array}$ & $\begin{array}{l}1, \mathrm{CC} \\
2, \mathrm{CC}\end{array}$ & $\begin{array}{l}14.09 \\
22.00\end{array}$ & & \\
\hline & & B & $\begin{array}{c}\text { Lamprocyrtis } \\
\text { nigriniae }\end{array}$ & $\begin{array}{l}3-3 \\
3, \mathrm{CC}\end{array}$ & $\begin{array}{l}15.81 \\
20.76\end{array}$ & $\begin{array}{l}1, \mathrm{CC} \\
2, \mathrm{CC}\end{array}$ & $\begin{array}{l}14.09 \\
22.00\end{array}$ & & \\
\hline & 1.35 & B & $\begin{array}{c}\text { Theocorythium } \\
\text { trachelium }\end{array}$ & $\begin{array}{l}3, C C \\
4-3\end{array}$ & $\begin{array}{l}20.76 \\
25.31\end{array}$ & $\begin{array}{l}1, \mathrm{CC} \\
2, \mathrm{CC}\end{array}$ & $\begin{array}{l}14.09 \\
22.00\end{array}$ & & \\
\hline \multirow[t]{2}{*}{ P. prismatium } & 1.5 & $\mathrm{~T}$ & $\begin{array}{l}\text { Pterocanium } \\
\text { prismatium }\end{array}$ & $\begin{array}{l}3, \mathrm{CC} \\
4-3\end{array}$ & $\begin{array}{l}20.76 \\
25.31\end{array}$ & $\begin{array}{l}2, \mathrm{CC} \\
3, \mathrm{CC} \\
\end{array}$ & $\begin{array}{l}22.00 \\
31.32 \\
\end{array}$ & & \\
\hline & & B & $\begin{array}{c}\text { Anthocyrtidium } \\
\text { angulare }\end{array}$ & $\begin{array}{l}4-3 \\
4-5\end{array}$ & $\begin{array}{l}25.31 \\
28.31\end{array}$ & $\begin{array}{l}2, \mathrm{CC} \\
3, \mathrm{CC}\end{array}$ & $\begin{array}{l}22.00 \\
31.32 \\
\end{array}$ & & \\
\hline \multirow[t]{9}{*}{ S. pentas } & 2.4 & $\mathbf{T}$ & $\begin{array}{l}\text { Stichocorys } \\
\text { peregrina }\end{array}$ & $\begin{array}{l}5-3 \\
5, C C\end{array}$ & $\begin{array}{l}34.61 \\
39.25\end{array}$ & $\begin{array}{l}3, \mathrm{CC} \\
4, \mathrm{CC}\end{array}$ & $\begin{array}{l}31.32 \\
39.73\end{array}$ & & \\
\hline & & $\mathrm{T}$ & $\begin{array}{l}\text { Phormostichoartus } \\
\text { fistula }\end{array}$ & $\begin{array}{l}6-3 \\
6, \mathrm{CC} \\
\end{array}$ & $\begin{array}{l}43.41 \\
48.01\end{array}$ & $\begin{array}{l}4, \mathrm{CC} \\
5, \mathrm{CC}\end{array}$ & $\begin{array}{l}39.73 \\
49.16 \\
\end{array}$ & & \\
\hline & 3.4 & $\mathrm{~T}$ & $\begin{array}{c}\text { Spongaster } \\
\text { pentas }\end{array}$ & $\begin{array}{l}6-3 \\
6, \mathrm{CC}\end{array}$ & $\begin{array}{l}43.41 \\
48.01\end{array}$ & & & & \\
\hline & & $\mathrm{T}$ & $\begin{array}{l}\text { Lychnodictyum } \\
\text { audax }\end{array}$ & {$\left[\begin{array}{l}7, \mathrm{CC} \\
8-3\end{array}\right.$} & $\left.\begin{array}{l}57.09 \\
60.70\end{array}\right]$ & $\begin{array}{l}4, C C \\
5, C C\end{array}$ & $\begin{array}{l}39.73 \\
49.16\end{array}$ & & \\
\hline & & $\mathrm{T}$ & $\begin{array}{c}\text { Phormostichoartus } \\
\text { doliolum }\end{array}$ & $\begin{array}{l}6, \mathrm{CC} \\
7-3 \\
\end{array}$ & $\begin{array}{l}48.01 \\
52.10\end{array}$ & $\begin{array}{l}5, \mathrm{CC} \\
6, \mathrm{CC}\end{array}$ & $\begin{array}{l}39.73 \\
49.16\end{array}$ & & \\
\hline & & B & $\begin{array}{l}\text { Amphirhopalum } \\
\text { ypsilon }\end{array}$ & $\begin{array}{l}7-3 \\
7, \mathrm{CC}\end{array}$ & $\begin{array}{l}52.10 \\
57.09\end{array}$ & & & & \\
\hline & & B & $\begin{array}{l}\text { Didymocyrtis } \\
\text { tetrathalamus }\end{array}$ & $\begin{array}{l}7-3 \\
7, \mathrm{CC} \\
\end{array}$ & $\begin{array}{l}52.10 \\
57.09\end{array}$ & & & & \\
\hline & 3.6 & B & $\begin{array}{c}\text { Spongaster } \\
\text { tetras }\end{array}$ & $\begin{array}{l}7-3 \\
7, \mathrm{CC} \\
\end{array}$ & $\begin{array}{l}52.10 \\
57.09\end{array}$ & & & & \\
\hline & 3.69 & $\mathrm{~T}$ & $\begin{array}{r}\text { Didymocyrtis } \\
\text { penultima }\end{array}$ & $\begin{array}{l}7-3 \\
7, \mathrm{CC}\end{array}$ & $\begin{array}{l}52.10 \\
57.09\end{array}$ & & & & \\
\hline
\end{tabular}


Table 2. (Continued).

\begin{tabular}{|c|c|c|c|c|c|c|c|c|c|}
\hline \multirow[b]{2}{*}{ Zone } & \multirow[b]{2}{*}{$\begin{array}{c}\text { Age } \\
\text { (Ma) }\end{array}$} & \multirow[b]{2}{*}{$\begin{array}{l}\text { Top } \\
\text { or } \\
\text { bottom }\end{array}$} & \multirow[b]{2}{*}{ Species } & \multicolumn{2}{|c|}{ Hole 573} & \multicolumn{2}{|c|}{ Hole 573A } & \multicolumn{2}{|c|}{ Hole 573B } \\
\hline & & & & Core-Section & $\begin{array}{l}\text { Sub-bottom } \\
\text { depth } \\
\text { (m) }\end{array}$ & Core-Section & $\begin{array}{l}\text { Sub-bottom } \\
\text { depth } \\
\text { (m) }\end{array}$ & Core-Section & $\begin{array}{l}\text { Sub-bottom } \\
\text { depth } \\
\text { (m) }\end{array}$ \\
\hline \multirow[t]{4}{*}{ S. pentas } & & $\mathrm{T}$ & $\begin{array}{r}\text { Solenosphaera } \\
\text { omnitubus }\end{array}$ & $\begin{array}{l}9-3 \\
9, \mathrm{CC}\end{array}$ & $\begin{array}{l}70.39 \\
76.18 \\
\end{array}$ & & & & \\
\hline & & B & $\begin{array}{l}\text { Didymocyrtis } \\
\text { avita }\end{array}$ & $\begin{array}{l}9-3 \\
9, \mathrm{CC}\end{array}$ & $\begin{array}{l}70.39 \\
76.18\end{array}$ & & & & \\
\hline & & $\mathrm{T}$ & $\begin{array}{l}\text { Spongaster } \\
\text { berminghami }\end{array}$ & {$\left[\begin{array}{l}8, \mathrm{CC} \\
9-3\end{array}\right.$} & $\left.\begin{array}{l}66.60 \\
70.39\end{array}\right]$ & & & & \\
\hline & & & $\begin{array}{l}S . \text { berminghami } \\
\rightarrow S \text {. pentas }\end{array}$ & $\begin{array}{l}8, \mathrm{CC} \\
10, \mathrm{CC}\end{array}$ & $\begin{array}{l}66.60 \\
85.76\end{array}$ & & & & \\
\hline \multirow[t]{10}{*}{ S. peregrina } & 4.3 & B & $\begin{array}{c}\text { Spongaster } \\
\text { pentas }\end{array}$ & $\begin{array}{l}9, \mathrm{CC} \\
10, \mathrm{CC}\end{array}$ & $\begin{array}{l}76.18 \\
85.76 \\
\end{array}$ & & & & \\
\hline & & $\mathrm{T}$ & $\begin{array}{r}\text { Botryostrobus } \\
\text { bramlettei }\end{array}$ & $\begin{array}{l}9, \mathrm{CC} \\
10-3\end{array}$ & $\begin{array}{l}76.18 \\
79.99\end{array}$ & & & & \\
\hline & 4.8 & B & $\begin{array}{l}\text { Pterocanium } \\
\text { prismatium }\end{array}$ & $\begin{array}{l}8, \mathrm{CC} \\
9-3\end{array}$ & $\begin{array}{l}66.60 \\
70.39\end{array}$ & & & & \\
\hline & & B & $\begin{array}{r}\text { Botryostrobus } \\
\text { aquilonaris }\end{array}$ & $\begin{array}{l}10-3 \\
10, \mathrm{CC}\end{array}$ & $\begin{array}{l}79.88 \\
85.76 \\
\end{array}$ & & & & \\
\hline & & $\mathrm{T}$ & $\begin{array}{l}\text { Didymocyrtis } \\
\text { antepenultima }\end{array}$ & {$\left[\begin{array}{l}11-3 \\
11, \mathrm{CC}\end{array}\right.$} & $\left.\begin{array}{l}89.34 \\
94.98\end{array}\right]$ & & & & \\
\hline & & $\mathrm{T}$ & $\begin{array}{l}\begin{array}{l}\text { Stichocorys } \\
\text { delmontensis }\end{array} \\
\end{array}$ & {$\left[\begin{array}{l}12-3 \\
12, \mathrm{CC}\end{array}\right.$} & $\left.\begin{array}{r}98.89 \\
104.15\end{array}\right]$ & & & & \\
\hline & & $\mathrm{T}$ & $\begin{array}{l}\text { Siphostichartus } \\
\text { corona }\end{array}$ & $\begin{array}{l}10-3 \\
10, \mathrm{CC}\end{array}$ & $\begin{array}{l}79.88 \\
85.76 \\
\end{array}$ & & & & \\
\hline & 5.0 & $\mathrm{~T}$ & $\begin{array}{c}\text { Acrobotrys } \\
\text { tritubus }\end{array}$ & $\begin{array}{l}11-3 \\
11, \mathrm{CC}\end{array}$ & $\begin{array}{l}89.34 \\
94.98 \\
\end{array}$ & & & & \\
\hline & & $\mathrm{T}$ & $\begin{array}{c}\text { Calocycletta } \\
\text { caepa }\end{array}$ & $\begin{array}{l}11-3 \\
11, \mathrm{CC}\end{array}$ & $\begin{array}{l}89.34 \\
94.98 \\
\end{array}$ & & & & \\
\hline & & & $\begin{array}{l}\text { S. delmontensis } \\
\rightarrow S \text {. peregrina }\end{array}$ & $\begin{array}{l}13-4 \\
13, \mathrm{CC}\end{array}$ & $\begin{array}{l}109.16 \\
112.57\end{array}$ & & & & \\
\hline \multirow[t]{3}{*}{ D. penultima } & & $\mathrm{T}$ & $\begin{array}{c}\text { Phormostichoartus } \\
\text { marylandicus }\end{array}$ & $\begin{array}{l}15-3 \\
15, \mathrm{CC}\end{array}$ & $\begin{array}{l}125.45 \\
131.29 \\
\end{array}$ & & & & \\
\hline & 6.55 & B & $\begin{array}{r}\text { Solenosphaera } \\
\text { omnitubus }\end{array}$ & $\begin{array}{l}15-3 \\
15, \mathrm{CC}\end{array}$ & $\begin{array}{l}125.45 \\
131.29 \\
\end{array}$ & & & & \\
\hline & {$[6.4]$} & B & $\begin{array}{l}\text { Stichocorys } \\
\text { peregrina }\end{array}$ & $\begin{array}{l}14-3 \\
14, \mathrm{CC} \\
\end{array}$ & $\begin{array}{l}116.06 \\
122.00 \\
\end{array}$ & & & & \\
\hline \multirow[t]{10}{*}{ D. antepenultima } & 8.2 & $\mathrm{~T}$ & $\begin{array}{l}\text { Diartus } \\
\text { hughesi }\end{array}$ & $\begin{array}{l}16-3 \\
16, \mathrm{CC} \\
\end{array}$ & $\begin{array}{l}134.91 \\
140.07 \\
\end{array}$ & & & & \\
\hline & & $\mathrm{T}$ & $\begin{array}{l}\text { Lithopera } \\
\text { neotera } \\
\end{array}$ & $\begin{array}{l}17-3 \\
17, \mathrm{CC}\end{array}$ & $\begin{array}{l}143.72 \\
148.99 \\
\end{array}$ & & & & \\
\hline & & $\mathrm{T}$ & $\begin{array}{c}\text { Dictyocoryne } \\
\text { ontongensis }\end{array}$ & $\begin{array}{l}17-3 \\
17, \mathrm{CC}\end{array}$ & $\begin{array}{l}143.72 \\
148.99 \\
\end{array}$ & & & $\begin{array}{l}1-1 \\
1, \mathrm{CC} \\
\end{array}$ & $\begin{array}{l}139.12 \\
147.01 \\
\end{array}$ \\
\hline & & $\mathbf{T}$ & $\begin{array}{l}\text { Botryocyrtis } \\
\quad \text { miralestensis }\end{array}$ & $\begin{array}{l}17-3 \\
17, \mathrm{CC}\end{array}$ & $\begin{array}{l}143.72 \\
148.99 \\
\end{array}$ & & & $\begin{array}{l}1, \mathrm{CC} \\
2-3\end{array}$ & $\begin{array}{l}147.01 \\
151.62 \\
\end{array}$ \\
\hline & [11.2] & B & $\begin{array}{c}\text { Acrobotrys } \\
\text { tritubus } \\
\end{array}$ & $\begin{array}{l}17-3 \\
17, \mathrm{CC} \\
\end{array}$ & $\begin{array}{l}143.72 \\
148.99 \\
\end{array}$ & & & $\begin{array}{l}1, \mathrm{CC} \\
2-3\end{array}$ & $\begin{array}{l}147.01 \\
151.62 \\
\end{array}$ \\
\hline & & $\mathrm{T}$ & $\begin{array}{r}\text { Didymocyrtis } \\
\text { laticonus }\end{array}$ & {$\left[\begin{array}{l}16-3 \\
16, \mathrm{CC}\end{array}\right.$} & $\left.\begin{array}{l}134.91 \\
140.07\end{array}\right]$ & & & {$\left[\begin{array}{l}1-1 \\
1, \mathrm{CC}\end{array}\right.$} & $\left.\begin{array}{l}139.12 \\
147.01\end{array}\right]$ \\
\hline & 8.2 & B & $\begin{array}{r}\text { Didymocyrtis } \\
\text { penultima }\end{array}$ & $\begin{array}{l}17, \mathrm{CC} \\
18, \mathrm{CC}\end{array}$ & $\begin{array}{l}148.99 \\
153.54 \\
\end{array}$ & & & $\begin{array}{l}1, \mathrm{CC} \\
2-3\end{array}$ & $\begin{array}{l}147.01 \\
151.62 \\
\end{array}$ \\
\hline & 9.7 & $\mathrm{~T}$ & $\begin{array}{l}\text { Diartus } \\
\quad \text { petterssoni }\end{array}$ & $\begin{array}{l}17, \mathrm{CC} \\
18, \mathrm{CC} \\
\end{array}$ & $\begin{array}{l}148.99 \\
153.54 \\
\end{array}$ & & & $\begin{array}{l}1, \mathrm{CC} \\
2-3\end{array}$ & $\begin{array}{l}147.01 \\
151.62 \\
\end{array}$ \\
\hline & & B & $\begin{array}{l}\text { Spongaster } \\
\quad \text { berminghami }\end{array}$ & {$\left[\begin{array}{l}17-3 \\
17, \mathrm{CC}\end{array}\right.$} & $\left.\begin{array}{l}143.72 \\
148.99\end{array}\right]$ & & & $\begin{array}{l}1, \mathrm{CC} \\
2-3\end{array}$ & $\begin{array}{l}147.01 \\
151.62 \\
\end{array}$ \\
\hline & & & $\begin{array}{l}\text { D. petterssoni } \\
\rightarrow \text { D. hughesi }\end{array}$ & $\begin{array}{l}18, \mathrm{CC} \\
19-2 \\
\end{array}$ & $\begin{array}{l}153.54 \\
155.02 \\
\end{array}$ & & & $\begin{array}{l}2-3 \\
2, \mathrm{CC} \\
\end{array}$ & $\begin{array}{l}151.62 \\
157.61 \\
\end{array}$ \\
\hline D. petterssoni & 11.5 & B & $\begin{array}{l}\text { Diartus } \\
\quad \text { hughesi }\end{array}$ & $\begin{array}{l}19-2 \\
19, \mathrm{CC}\end{array}$ & $\begin{array}{l}155.02 \\
159.07\end{array}$ & & & $\begin{array}{l}2-3 \\
2, \mathrm{CC}\end{array}$ & $\begin{array}{l}151.62 \\
157.61 \\
\end{array}$ \\
\hline
\end{tabular}


Table 2. (Continued).

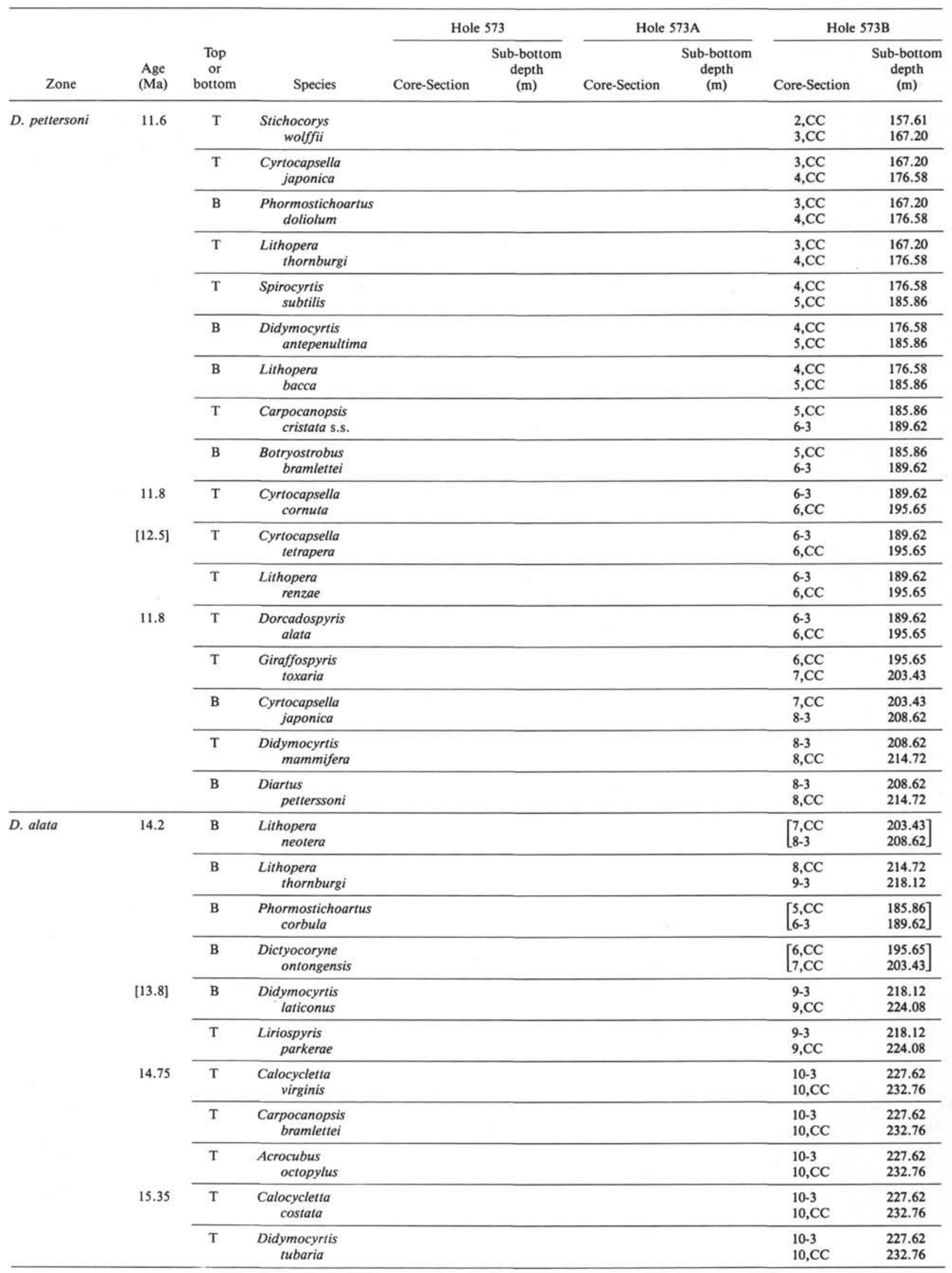




\section{A. NIGRINI}

Table 2. (Continued).

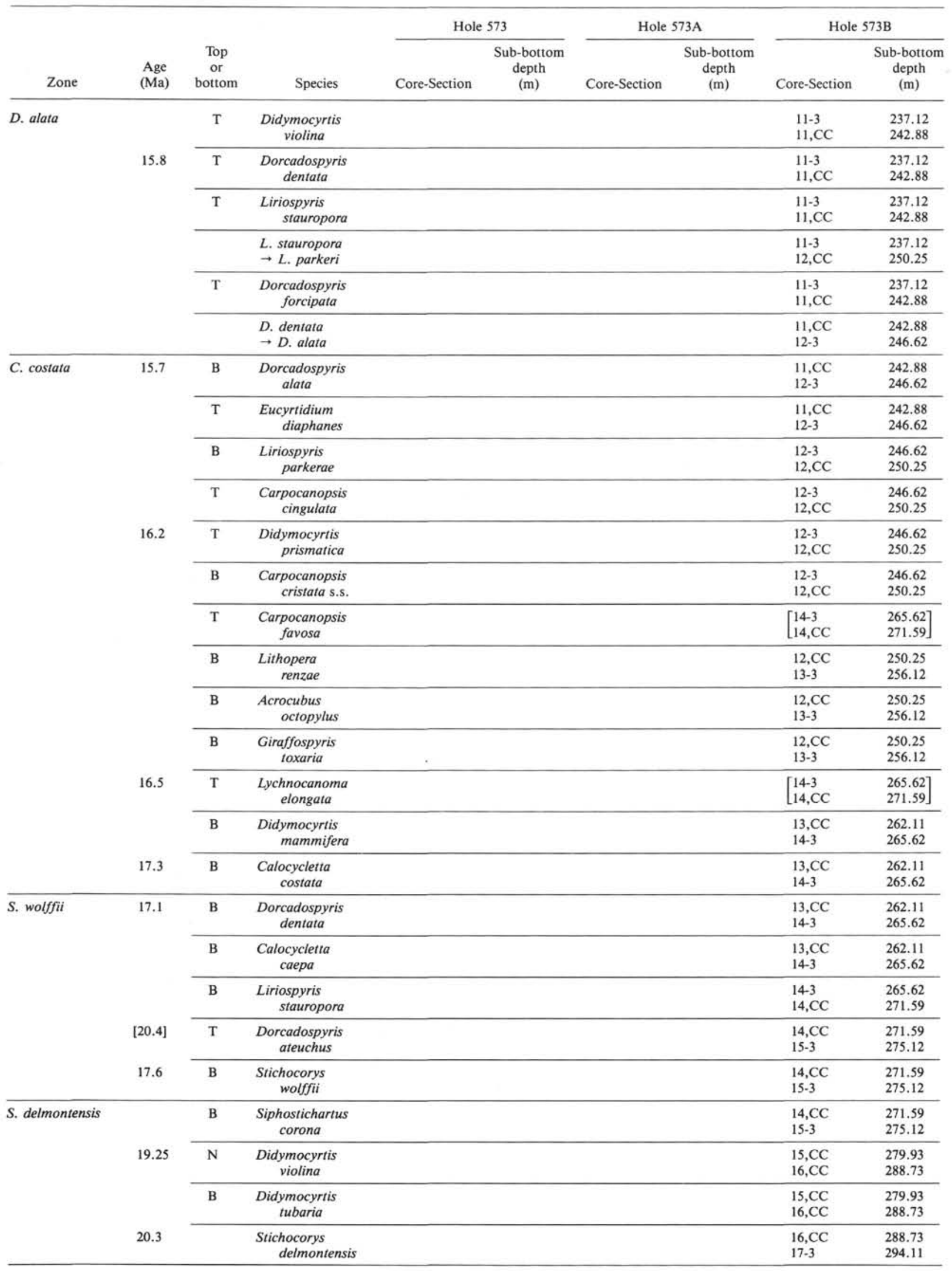


Table 2. (Continued).

\begin{tabular}{|c|c|c|c|c|c|c|c|c|c|}
\hline \multirow[b]{2}{*}{ Zone } & \multirow[b]{2}{*}{$\begin{array}{c}\text { Age } \\
(\mathrm{Ma})\end{array}$} & \multirow[b]{2}{*}{$\begin{array}{c}\text { Top } \\
\text { or } \\
\text { bottom }\end{array}$} & \multirow[b]{2}{*}{ Species } & \multicolumn{2}{|c|}{ Hole 573} & \multicolumn{2}{|c|}{ Hole $573 \mathrm{~A}$} & \multicolumn{2}{|c|}{ Hole 573B } \\
\hline & & & & Core-Section & $\begin{array}{l}\text { Sub-bottom } \\
\text { depth } \\
\text { (m) }\end{array}$ & Core-Section & $\begin{array}{l}\text { Sub-bottom } \\
\text { depth } \\
\text { (m) }\end{array}$ & Core-Section & $\begin{array}{l}\text { Sub-bottom } \\
\text { depth } \\
\text { (m) }\end{array}$ \\
\hline S. delmontensis & & B & $\begin{array}{c}\text { Carpocanopsis } \\
\text { bramlettei }\end{array}$ & & & & & $\begin{array}{l}16, \mathrm{CC} \\
17-3\end{array}$ & $\begin{array}{l}288.73 \\
294.11\end{array}$ \\
\hline \multirow[t]{8}{*}{ C. tetrapera } & \multirow[t]{2}{*}{21.3} & $\mathrm{~T}$ & $\begin{array}{r}\text { Theocyrtis } \\
\text { annosa }\end{array}$ & & & & & $\begin{array}{l}16, \mathrm{CC} \\
17-3\end{array}$ & $\begin{array}{l}288.73 \\
294.11\end{array}$ \\
\hline & & $\mathrm{T}$ & $\begin{array}{c}\text { Calocycletta } \\
\text { robusta }\end{array}$ & & & & & $\begin{array}{l}16, \mathrm{CC} \\
17-3\end{array}$ & $\begin{array}{l}288.73 \\
294.11\end{array}$ \\
\hline & \multirow[t]{4}{*}{21.35} & B & $\begin{array}{c}\text { Calocycletta } \\
\text { virginis }\end{array}$ & & & & & $\begin{array}{l}16, \mathrm{CC} \\
17-3\end{array}$ & $\begin{array}{l}288.73 \\
294.11\end{array}$ \\
\hline & & $\mathrm{T}$ & $\begin{array}{c}\text { Cyclampterium } \\
\text { pegetrum }\end{array}$ & & & & & $\begin{array}{l}17-3 \\
17, \mathrm{CC}\end{array}$ & $\begin{array}{l}294.11 \\
298.24\end{array}$ \\
\hline & & B & $\begin{array}{l}\text { Botryostrobus } \\
\quad \text { miralestensis }\end{array}$ & & & & & $\begin{array}{l}17, \mathrm{CC} \\
18, \mathrm{CC}\end{array}$ & $\begin{array}{l}298.24 \\
304.62\end{array}$ \\
\hline & & B & $\begin{array}{c}\text { Carpocanopsis } \\
\text { cingulata }\end{array}$ & & & & & $\begin{array}{l}18, \mathrm{CC} \\
19, \mathrm{CC}\end{array}$ & $\begin{array}{l}304.62 \\
312.74\end{array}$ \\
\hline & 22.2 & B & $\begin{array}{c}\text { Cyrtocapsella } \\
\text { cornuta }\end{array}$ & & & & & $\begin{array}{l}18, \mathrm{CC} \\
19, \mathrm{CC}\end{array}$ & $\begin{array}{l}304.62 \\
312.74\end{array}$ \\
\hline & 22.2 & B & $\begin{array}{c}\text { Cyrtocapsella } \\
\text { tetrapera }\end{array}$ & & & & & $\begin{array}{l}18, \mathrm{CC} \\
19, \mathrm{CC} \\
\end{array}$ & $\begin{array}{l}304.62 \\
312.74 \\
\end{array}$ \\
\hline \multirow[t]{3}{*}{ L. elongata } & & B & $\begin{array}{l}\text { Carpocanopsis } \\
\text { favosa }\end{array}$ & & & & & $\begin{array}{l}18, \mathrm{CC} \\
19, \mathrm{CC}\end{array}$ & $\begin{array}{l}304.62 \\
312.74\end{array}$ \\
\hline & 22.5 & $\mathrm{~T}$ & $\begin{array}{c}\text { Artophormis } \\
\text { gracilis }\end{array}$ & & & & & $\begin{array}{l}18, \mathrm{CC} \\
19, \mathrm{CC}\end{array}$ & $\begin{array}{l}304.62 \\
312.74 \\
\end{array}$ \\
\hline & 25.9 & B & $\begin{array}{c}\text { Lychnocanoma } \\
\text { elongata }\end{array}$ & & & & & $\begin{array}{l}20, \mathrm{CC} \\
21, \mathrm{CC}\end{array}$ & $\begin{array}{l}328.25 \\
334.99\end{array}$ \\
\hline \multirow[t]{9}{*}{ D. ateuchus } & & B & $\begin{array}{c}\text { Calocycletta } \\
\text { robusta }\end{array}$ & & & & & $\begin{array}{l}21, \mathrm{CC} \\
22, \mathrm{CC}\end{array}$ & $\begin{array}{l}334.99 \\
343.99 \\
\end{array}$ \\
\hline & & B & $\begin{array}{c}\text { Dorcadospyris } \\
\text { forcipata }\end{array}$ & & & & & $\begin{array}{l}26, \mathrm{CC} \\
27, \mathrm{CC}\end{array}$ & $\begin{array}{l}383.71 \\
392.46 \\
\end{array}$ \\
\hline & & $\mathrm{T}$ & $\begin{array}{l}\text { Lithocyclis } \\
\text { angusta }\end{array}$ & & & & & $\begin{array}{l}29, \mathrm{CC} \\
30, \mathrm{CC} \\
\end{array}$ & $\begin{array}{l}411.87 \\
420.38 \\
\end{array}$ \\
\hline & & B & $\begin{array}{r}\text { Theocyrtis } \\
\text { annosa }\end{array}$ & & & & & $\begin{array}{l}30, \mathrm{CC} \\
31, \mathrm{CC}\end{array}$ & $\begin{array}{l}420.38 \\
437.50 \\
\end{array}$ \\
\hline & & $\mathrm{T}$ & $\begin{array}{l}\text { Lithocyclis } \\
\text { crux }\end{array}$ & & & & & $\begin{array}{l}33, \mathrm{CC} \\
34, \mathrm{CC} \\
\end{array}$ & $\begin{array}{l}450.68 \\
454.43 \\
\end{array}$ \\
\hline & & B & $\begin{array}{r}\text { Didymocyrtis } \\
\text { prismatica }\end{array}$ & & & & & {$\left[\begin{array}{l}34, \mathrm{CC} \\
35, \mathrm{CC}\end{array}\right.$} & $\left.\begin{array}{l}454.43 \\
468.95\end{array}\right]$ \\
\hline & & $\mathrm{T}$ & $\begin{array}{c}\text { Cyclampterium } \\
\text { milowi }\end{array}$ & & & & & $\begin{array}{l}33, \mathrm{CC} \\
34, \mathrm{CC} \\
\end{array}$ & $\begin{array}{l}450.68 \\
454.43 \\
\end{array}$ \\
\hline & & $\mathrm{T}$ & $\begin{array}{c}\text { Tristylospyris } \\
\text { triceros }\end{array}$ & & & & & $\begin{array}{l}33, \mathrm{CC} \\
34, \mathrm{CC} \\
\end{array}$ & $\begin{array}{l}450.68 \\
454.43 \\
\end{array}$ \\
\hline & & & $\begin{array}{l}\text { T. triceros } \\
\rightarrow \text { D. ateuchus }\end{array}$ & & & & & $\begin{array}{l}33, \mathrm{CC} \\
35, \mathrm{CC}\end{array}$ & $\begin{array}{l}450.68 \\
468.95 \\
\end{array}$ \\
\hline \multirow[t]{5}{*}{ T. tuberosa } & 33.0 & B & $\begin{array}{c}\text { Dorcadospyris } \\
\text { ateuchus }\end{array}$ & & & & & $\begin{array}{l}33, \mathrm{CC} \\
34, \mathrm{CC}\end{array}$ & $\begin{array}{l}450.68 \\
454.43 \\
\end{array}$ \\
\hline & & B & $\begin{array}{c}\text { Cyclampterium } \\
\text { pegetrum }\end{array}$ & & & & & $\begin{array}{l}34, \mathrm{CC} \\
35, \mathrm{CC}\end{array}$ & $\begin{array}{l}454.43 \\
468.95\end{array}$ \\
\hline & & $\mathrm{T}$ & $\begin{array}{l}\text { Theocyrtis } \\
\text { tuberosa }\end{array}$ & & & & & $\begin{array}{l}34, \mathrm{CC} \\
35, \mathrm{CC}\end{array}$ & $\begin{array}{l}454.43 \\
468.95 \\
\end{array}$ \\
\hline & & B & $\begin{array}{l}\text { Eucyrtidium } \\
\text { diaphanes }\end{array}$ & & & & & $\begin{array}{l}34, \mathrm{CC} \\
35, \mathrm{CC}\end{array}$ & $\begin{array}{l}454.43 \\
468.95\end{array}$ \\
\hline & & B & $\begin{array}{r}\text { Lithocyclia } \\
\text { angusta }\end{array}$ & & & & & $\begin{array}{l}37, \mathrm{CC} \\
38, \mathrm{CC}\end{array}$ & $\begin{array}{l}482.28 \\
494.41\end{array}$ \\
\hline
\end{tabular}

Note: Pairs of levels and brackets as defined in Table 1 of this appendix. 
Table 3. List of radiolarian events for Site 574.

\begin{tabular}{|c|c|c|c|c|c|c|c|c|c|}
\hline \multirow[b]{2}{*}{ Zone } & \multirow[b]{2}{*}{$\begin{array}{l}\text { Age } \\
\text { (Ma) }\end{array}$} & \multirow[b]{2}{*}{$\begin{array}{l}\text { Top } \\
\text { or } \\
\text { bottom }\end{array}$} & \multirow[b]{2}{*}{ Species } & \multicolumn{2}{|c|}{ Hole 574} & \multicolumn{2}{|c|}{ Hole 574A } & \multicolumn{2}{|c|}{ Hole $574 \mathrm{C}$} \\
\hline & & & & Core-Section & $\begin{array}{l}\text { Sub-bottom } \\
\text { depth } \\
\text { (m) }\end{array}$ & Core-Section & $\begin{array}{l}\text { Sub-bottom } \\
\text { depth } \\
\text { (m) }\end{array}$ & Core-Section & $\begin{array}{l}\text { Sub-bottom } \\
\text { depth } \\
\text { (m) }\end{array}$ \\
\hline B. invaginata & 0.3 & B & $\begin{array}{c}\text { Buccinosphaera } \\
\text { invaginata }\end{array}$ & & & & & & \\
\hline \multirow[t]{3}{*}{ C. tuberosa } & 0.44 & $\mathrm{~T}$ & $\begin{array}{l}\text { Stylatractus } \\
\text { universus }\end{array}$ & $\begin{array}{l}1-2 \\
1, \mathrm{CC}\end{array}$ & $\begin{array}{l}2.41 \\
4.48\end{array}$ & $1, \mathrm{CC}$ & $\begin{array}{l}0.00 \\
6.30\end{array}$ & & \\
\hline & & B & $\begin{array}{c}\text { Collosphaera } \\
\text { tuberosa }\end{array}$ & $\begin{array}{l}1-2 \\
1, \mathrm{CC}\end{array}$ & $\begin{array}{l}2.41 \\
4.48\end{array}$ & & & & \\
\hline & & B & $\begin{array}{l}\text { Pterocorys } \\
\text { hertwigii }\end{array}$ & $\begin{array}{l}1, \mathrm{CC} \\
2-1\end{array}$ & $\begin{array}{l}4.48 \\
5.25\end{array}$ & $\begin{array}{l}1, C C \\
2, C C\end{array}$ & $\begin{array}{r}6.30 \\
15.45 \\
\end{array}$ & & \\
\hline A. ypsilon & 1.1 & $\mathbf{T}$ & $\begin{array}{c}\text { Anthocyrtidium } \\
\text { angulare }\end{array}$ & $\begin{array}{l}1, \mathrm{CC} \\
2-1\end{array}$ & $\begin{array}{l}4.48 \\
5.25\end{array}$ & & & & \\
\hline \multirow[t]{4}{*}{ A. angulare } & & $\mathbf{T}$ & $\begin{array}{c}\text { Theocorythium } \\
\text { vetulum }\end{array}$ & $\begin{array}{l}1, \mathrm{CC} \\
2-1\end{array}$ & $\begin{array}{l}4.48 \\
5.25\end{array}$ & $\begin{array}{l}1, C C \\
2, C C\end{array}$ & $\begin{array}{r}6.30 \\
15.45\end{array}$ & & \\
\hline & & $\mathrm{T}$ & $\begin{array}{l}\text { Didymocyrtis } \\
\text { avita }\end{array}$ & $\begin{array}{l}1, \mathrm{CC} \\
2-1\end{array}$ & $\begin{array}{l}4.48 \\
5.25\end{array}$ & & & & \\
\hline & & B & $\begin{array}{c}\text { Lamprocyrtis } \\
\text { nigriniae }\end{array}$ & $\begin{array}{l}1, \mathrm{CC} \\
2-1\end{array}$ & $\begin{array}{l}4.48 \\
5.25\end{array}$ & $\begin{array}{l}1, \mathrm{CC} \\
2, \mathrm{CC}\end{array}$ & $\begin{array}{r}6.30 \\
15.45 \\
\end{array}$ & & \\
\hline & 1.35 & B & $\begin{array}{c}\text { Theocorythium } \\
\text { trachelium }\end{array}$ & $\begin{array}{l}2-2 \\
2-4 \\
\end{array}$ & $\begin{array}{l}6.75 \\
9.75 \\
\end{array}$ & $\begin{array}{l}1, \mathrm{CC} \\
2, \mathrm{CC}\end{array}$ & $\begin{array}{r}6.30 \\
15.45 \\
\end{array}$ & & \\
\hline \multirow[t]{2}{*}{ P. prismatium } & 1.5 & $\mathrm{~T}$ & $\begin{array}{l}\text { Pterocanium } \\
\text { prismatium }\end{array}$ & $\begin{array}{l}2-2 \\
2-3 \\
\end{array}$ & $\begin{array}{l}6.75 \\
8.25 \\
\end{array}$ & $\begin{array}{l}1, \mathrm{CC} \\
2, \mathrm{CC}\end{array}$ & $\begin{array}{r}6.30 \\
15.45 \\
\end{array}$ & & \\
\hline & & B & $\begin{array}{c}\text { Anthocyrtidium } \\
\text { angulare }\end{array}$ & $\begin{array}{l}2-5 \\
2-6 \\
\end{array}$ & $\begin{array}{l}11.25 \\
12.75 \\
\end{array}$ & & & & \\
\hline \multirow[t]{13}{*}{ S. pentas } & 2.4 & $\mathrm{~T}$ & $\begin{array}{c}\text { Stichocorys } \\
\text { peregrina }\end{array}$ & $\begin{array}{l}2-5 \\
2-6 \\
\end{array}$ & $\begin{array}{l}11.25 \\
12.75 \\
\end{array}$ & $\begin{array}{l}1, \mathrm{CC} \\
2, \mathrm{CC}\end{array}$ & $\begin{array}{r}6.30 \\
15.45 \\
\end{array}$ & & \\
\hline & & $\mathrm{T}$ & $\begin{array}{l}\text { Phormostichoartus } \\
\quad \text { fistula }\end{array}$ & $\begin{array}{l}2, \mathrm{CC} \\
3-4\end{array}$ & $\begin{array}{l}14.07 \\
19.20\end{array}$ & {$\left[\begin{array}{l}3, \mathrm{CC} \\
4, \mathrm{CC}\end{array}\right.$} & $\left.\begin{array}{l}23.92 \\
33.13\end{array}\right]$ & & \\
\hline & 3.4 & $\mathrm{~T}$ & $\begin{array}{c}\text { Spongaster } \\
\text { pentas }\end{array}$ & $\begin{array}{l}2, \mathrm{CC} \\
3-4\end{array}$ & $\begin{array}{l}14.07 \\
19.20\end{array}$ & $\begin{array}{l}2, \mathrm{CC} \\
3, \mathrm{CC}\end{array}$ & $\begin{array}{l}15.45 \\
23.92\end{array}$ & & \\
\hline & & $\mathrm{T}$ & $\begin{array}{l}\text { Lychnodictyum } \\
\text { audax }\end{array}$ & {$\left[\begin{array}{l}3-4 \\
3, \mathrm{CC}\end{array}\right.$} & $\left.\begin{array}{l}19.20 \\
23.48\end{array}\right]$ & {$\left[\begin{array}{l}4, \mathrm{CC} \\
5, \mathrm{CC}\end{array}\right.$} & $\left.\begin{array}{l}33.13 \\
42.34\end{array}\right]$ & & \\
\hline & & $\mathrm{T}$ & $\begin{array}{l}\text { Phormostichoartus } \\
\text { doliolum }\end{array}$ & $\begin{array}{l}2, \mathrm{CC} \\
3-4\end{array}$ & $\begin{array}{l}14.07 \\
19.20\end{array}$ & $\begin{array}{l}2, \mathrm{CC} \\
3, \mathrm{CC}\end{array}$ & $\begin{array}{l}15.45 \\
23.92\end{array}$ & & \\
\hline & & B & $\begin{array}{l}\text { Amphirhopalum } \\
\text { ypsilon }\end{array}$ & $\begin{array}{l}3-4 \\
3, \mathrm{CC}\end{array}$ & $\begin{array}{l}19.20 \\
23.48\end{array}$ & $\begin{array}{l}2, \mathrm{CC} \\
3, \mathrm{CC}\end{array}$ & $\begin{array}{l}15.45 \\
23.92 \\
\end{array}$ & & \\
\hline & & B & $\begin{array}{c}\text { Didymocyrtis } \\
\text { tetrathalamus }\end{array}$ & $\begin{array}{l}3-4 \\
3, \mathrm{CC}\end{array}$ & $\begin{array}{l}19.20 \\
23.48 \\
\end{array}$ & $\begin{array}{l}2, \mathrm{CC} \\
3, \mathrm{CC}\end{array}$ & $\begin{array}{l}15.45 \\
23.92 \\
\end{array}$ & & \\
\hline & 3.6 & B & $\begin{array}{c}\text { Spongaster } \\
\text { tetras }\end{array}$ & $\begin{array}{l}3-4 \\
3, \mathrm{CC}\end{array}$ & $\begin{array}{l}19.20 \\
23.48 \\
\end{array}$ & $\begin{array}{l}2, \mathrm{CC} \\
3, \mathrm{CC} \\
\end{array}$ & $\begin{array}{l}15.45 \\
23.92 \\
\end{array}$ & & \\
\hline & 3.69 & $\mathrm{~T}$ & $\begin{array}{r}\text { Didymocyrtis } \\
\text { penultima }\end{array}$ & $\begin{array}{l}3-4 \\
3, \mathrm{CC}\end{array}$ & $\begin{array}{l}19.20 \\
23.48\end{array}$ & $\begin{array}{l}2, \mathrm{CC} \\
3, \mathrm{CC}\end{array}$ & $\begin{array}{l}15.45 \\
23.92 \\
\end{array}$ & & \\
\hline & & $\mathrm{T}$ & $\begin{array}{r}\text { Solenosphaera } \\
\text { omnitubus }\end{array}$ & $\begin{array}{l}3-4 \\
3, \mathrm{CC}\end{array}$ & $\begin{array}{l}19.20 \\
23.48\end{array}$ & $\begin{array}{l}3, \mathrm{CC} \\
4, \mathrm{CC}\end{array}$ & $\begin{array}{l}23.92 \\
33.13 \\
\end{array}$ & $\cdot$ & \\
\hline & & B & $\begin{array}{c}\text { Didymocyrtis } \\
\text { avita }\end{array}$ & $\begin{array}{l}3-4 \\
3, \mathrm{CC}\end{array}$ & $\begin{array}{l}19.20 \\
23.48 \\
\end{array}$ & & -1 & & \\
\hline & & $\mathrm{T}$ & $\begin{array}{l}\text { Spongaster } \\
\text { berminghami }\end{array}$ & $\begin{array}{l}4-4 \\
4, \mathrm{CC}\end{array}$ & $\begin{array}{l}28.73 \\
33.10 \\
\end{array}$ & $\begin{array}{l}3, \mathrm{CC} \\
4, \mathrm{CC}\end{array}$ & $\begin{array}{l}23.92 \\
33.13 \\
\end{array}$ & & \\
\hline & & & $\begin{array}{l}\text { S. berminghami } \\
\rightarrow \text { S. pentas }\end{array}$ & $\begin{array}{l}4, \mathrm{CC} \\
5-1 \\
\end{array}$ & $\begin{array}{r}33.10 \\
33.44 \\
\end{array}$ & $\begin{array}{l}4, \mathrm{CC} \\
5, \mathrm{CC}\end{array}$ & $\begin{array}{l}33.13 \\
42.34 \\
\end{array}$ & & \\
\hline \multirow[t]{4}{*}{ S. peregrina } & 4.3 & B & $\begin{array}{c}\text { Spongaster } \\
\text { pentas }\end{array}$ & $\begin{array}{l}5-1 \\
5-2\end{array}$ & $\begin{array}{l}33.44 \\
34.94 \\
\end{array}$ & $\begin{array}{l}4, \mathrm{CC} \\
5, \mathrm{CC}\end{array}$ & $\begin{array}{l}33.13 \\
42.34 \\
\end{array}$ & & \\
\hline & 4.8 & $\begin{array}{l}\mathrm{T} \\
\mathrm{B}\end{array}$ & $\begin{array}{c}\begin{array}{c}\text { Botryostrobus } \\
\text { bramlettei } \\
\text { Pterocanium } \\
\text { prismatium }\end{array} \\
\end{array}$ & $\begin{array}{l}{\left[\begin{array}{l}3, \mathrm{CC} \\
4-4\end{array}\right.} \\
{\left[\begin{array}{l}5-2 \\
5-4\end{array}\right.}\end{array}$ & $\left.\begin{array}{l}23.48 \\
28.73 \\
34.94 \\
37.94\end{array}\right]$ & 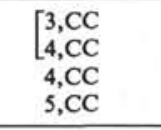 & $\begin{array}{l}23.92 \\
33.13 \\
33.13 \\
42.34 \\
\end{array}$ & & \\
\hline & & B & $\begin{array}{c}\text { Botryostrobus } \\
\text { aquilonaris }\end{array}$ & $\begin{array}{l}5-1 \\
5-2\end{array}$ & $\begin{array}{l}33.44 \\
34.94\end{array}$ & $\begin{array}{l}4, \mathrm{CC} \\
5, \mathrm{CC}\end{array}$ & $\begin{array}{l}33.13 \\
42.34\end{array}$ & & \\
\hline & & $\mathrm{T}$ & $\begin{array}{l}\text { Didymocyrtis } \\
\quad \text { antepenultima }\end{array}$ & $\begin{array}{l}4, \mathrm{CC} \\
5-2\end{array}$ & $\begin{array}{l}33.10 \\
34.94\end{array}$ & $\begin{array}{l}4, C C \\
5, C C\end{array}$ & $\begin{array}{l}33.13 \\
42.34 \\
\end{array}$ & & \\
\hline
\end{tabular}


Table 3. (Continued).

\begin{tabular}{|c|c|c|c|c|c|c|c|c|c|}
\hline \multirow[b]{2}{*}{ Zone } & \multirow[b]{2}{*}{$\begin{array}{l}\text { Age } \\
(\mathrm{Ma})\end{array}$} & \multirow[b]{2}{*}{$\begin{array}{c}\text { Top } \\
\text { or } \\
\text { bottom }\end{array}$} & \multirow[b]{2}{*}{ Species } & \multicolumn{2}{|c|}{ Hole 574} & \multicolumn{2}{|c|}{ Hole 574A } & \multicolumn{2}{|c|}{ Hole 574B } \\
\hline & & & & Core-Section & $\begin{array}{l}\text { Sub-bottom } \\
\text { depth } \\
\text { (m) }\end{array}$ & Core-Section & $\begin{array}{l}\text { Sub-bottom } \\
\text { depth } \\
\text { (m) }\end{array}$ & Core-Section & $\begin{array}{l}\text { Sub-bottom } \\
\text { depth } \\
\text { (m) }\end{array}$ \\
\hline \multirow[t]{5}{*}{ S. peregrina } & \multirow{5}{*}{5.0} & $\mathrm{~T}$ & $\begin{array}{l}\text { Stichocorys } \\
\quad \text { delmontensis }\end{array}$ & $\begin{array}{l}4, C C \\
5-4\end{array}$ & $\begin{array}{l}33.10 \\
37.94 \\
\end{array}$ & $\begin{array}{l}4, C C \\
5, C C\end{array}$ & $\begin{array}{l}33.13 \\
42.34\end{array}$ & & \\
\hline & & $\mathrm{T}$ & $\begin{array}{l}\text { Siphostichartus } \\
\text { corona }\end{array}$ & $\begin{array}{l}4, C C \\
5-1\end{array}$ & $\begin{array}{l}33.10 \\
33.44\end{array}$ & $\begin{array}{l}4, C C \\
5, \mathrm{CC}\end{array}$ & $\begin{array}{l}33.13 \\
42.34\end{array}$ & & \\
\hline & & $\mathrm{T}$ & $\begin{array}{c}\text { Acrobotrys } \\
\text { tritubus }\end{array}$ & $\begin{array}{l}4, C C \\
5-4\end{array}$ & $\begin{array}{l}33.10 \\
37.94\end{array}$ & $\begin{array}{l}4, C C \\
5, C C\end{array}$ & $\begin{array}{l}33.13 \\
42.34\end{array}$ & & \\
\hline & & $\mathrm{T}$ & $\begin{array}{c}\text { Calocycletta } \\
\text { caepa }\end{array}$ & $\begin{array}{l}5, \mathrm{CC} \\
6-2\end{array}$ & $\begin{array}{l}42.54 \\
44.42\end{array}$ & $\begin{array}{l}5, C C \\
6, C C\end{array}$ & $\begin{array}{l}42.34 \\
51.92 \\
\end{array}$ & & \\
\hline & & & $\begin{array}{l}S . \text { delmontensis } \\
\rightarrow S \text {. peregrina }\end{array}$ & $\begin{array}{l}6-2 \\
6, \mathrm{CC}\end{array}$ & $\begin{array}{l}44.42 \\
52.00\end{array}$ & $\begin{array}{l}5, C C \\
6, C C\end{array}$ & $\begin{array}{l}42.34 \\
51.92 \\
\end{array}$ & & \\
\hline \multirow[t]{3}{*}{ D. penultima } & & $\mathrm{T}$ & $\begin{array}{c}\text { Phormostichoartus } \\
\text { marylandicus }\end{array}$ & $\begin{array}{l}6-2 \\
6, \mathrm{CC} \\
\end{array}$ & $\begin{array}{l}44.42 \\
52.00 \\
\end{array}$ & $\begin{array}{l}6, \mathrm{CC} \\
7, \mathrm{CC} \\
\end{array}$ & $\begin{array}{l}51.92 \\
61.26 \\
\end{array}$ & & \\
\hline & 6.55 & B & $\begin{array}{r}\text { Solenosphaera } \\
\text { omnitubus }\end{array}$ & $\begin{array}{l}6, \mathrm{CC} \\
7-4 \\
\end{array}$ & $\begin{array}{l}52.00 \\
56.92 \\
\end{array}$ & $\begin{array}{l}6, \mathrm{CC} \\
7, \mathrm{CC} \\
\end{array}$ & $\begin{array}{l}51.92 \\
61.26 \\
\end{array}$ & 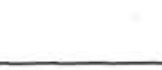 & \\
\hline & {$[6.4]$} & B & $\begin{array}{c}\text { Stichocorys } \\
\text { peregrina }\end{array}$ & $\begin{array}{l}6, \mathrm{CC} \\
7-4 \\
\end{array}$ & $\begin{array}{l}52.00 \\
56.92 \\
\end{array}$ & $\begin{array}{l}6, \mathrm{CC} \\
7, \mathrm{CC} \\
\end{array}$ & $\begin{array}{l}51.92 \\
61.26 \\
\end{array}$ & & \\
\hline \multirow[t]{10}{*}{ D. antepenultima } & 8.2 & $\mathbf{T}$ & $\begin{array}{l}\text { Diartus } \\
\quad \text { hughesi }\end{array}$ & $\begin{array}{l}7-4 \\
7, \mathrm{CC} \\
\end{array}$ & $\begin{array}{l}56.92 \\
60.93 \\
\end{array}$ & $\begin{array}{l}6, \mathrm{CC} \\
7, \mathrm{CC} \\
\end{array}$ & $\begin{array}{l}51.92 \\
61.26 \\
\end{array}$ & & \\
\hline & & $\mathbf{T}$ & $\begin{array}{c}\text { Lithopera } \\
\text { neotera }\end{array}$ & $\begin{array}{l}7-4 \\
7, \mathrm{CC} \\
\end{array}$ & $\begin{array}{l}56.92 \\
60.93 \\
\end{array}$ & $\begin{array}{l}6, \mathrm{CC} \\
7, \mathrm{CC} \\
\end{array}$ & $\begin{array}{l}51.92 \\
61.26 \\
\end{array}$ & & \\
\hline & & $\mathrm{T}$ & $\begin{array}{l}\text { Dictyocoryne } \\
\text { ontongensis }\end{array}$ & $\begin{array}{l}7-4 \\
7, \mathrm{CC}\end{array}$ & $\begin{array}{l}56.92 \\
60.93 \\
\end{array}$ & $\begin{array}{l}6, \mathrm{CC} \\
7, \mathrm{CC} \\
\end{array}$ & $\begin{array}{l}51.92 \\
61.26 \\
\end{array}$ & & \\
\hline & & $\mathrm{T}$ & $\begin{array}{l}\text { Botryostrobus } \\
\text { miralestensis }\end{array}$ & $\begin{array}{l}7, \mathrm{CC} \\
8-4 \\
\end{array}$ & $\begin{array}{l}60.93 \\
65.92 \\
\end{array}$ & $\begin{array}{l}7, \mathrm{CC} \\
8, \mathrm{CC}\end{array}$ & $\begin{array}{l}61.26 \\
70.42 \\
\end{array}$ & & \\
\hline & {$[11.2]$} & B & $\begin{array}{r}\text { Acrobotrys } \\
\text { tritubus }\end{array}$ & $\begin{array}{l}7, C C \\
8-4 \\
\end{array}$ & $\begin{array}{l}60.93 \\
65.92 \\
\end{array}$ & $\begin{array}{l}7, C C \\
8, C C \\
\end{array}$ & $\begin{array}{l}61.26 \\
70.42 \\
\end{array}$ & & \\
\hline & & $\mathbf{T}$ & $\begin{array}{c}\text { Didymocyrtis } \\
\text { laticonus }\end{array}$ & {$\left[\begin{array}{l}7-4 \\
7, \mathrm{CC} \\
\end{array}\right.$} & $\left.\begin{array}{l}56.92 \\
60.93\end{array}\right]$ & $\begin{array}{l}7, \mathrm{CC} \\
8, \mathrm{CC} \\
\end{array}$ & $\begin{array}{l}61.26 \\
70.42 \\
\end{array}$ & & \\
\hline & 8.2 & B & $\begin{array}{r}\text { Didymocyrtis } \\
\text { penultima }\end{array}$ & $\begin{array}{l}8-4 \\
8, C C\end{array}$ & $\begin{array}{l}65.92 \\
70.52 \\
\end{array}$ & $\begin{array}{l}7, C C \\
8, C C \\
\end{array}$ & $\begin{array}{l}61.26 \\
70.42 \\
\end{array}$ & & \\
\hline & 9.7 & $\mathbf{T}$ & $\begin{array}{l}\text { Diartus } \\
\quad \text { petterssoni }\end{array}$ & $\begin{array}{l}8-4 \\
8, C C\end{array}$ & $\begin{array}{l}65.92 \\
70.52 \\
\end{array}$ & $\begin{array}{l}7, \mathrm{CC} \\
8, \mathrm{CC} \\
\end{array}$ & $\begin{array}{l}61.26 \\
70.42 \\
\end{array}$ & & \\
\hline & & B & $\begin{array}{l}\text { Spongaster } \\
\text { berminghami }\end{array}$ & $\begin{array}{l}8-4 \\
8, \mathrm{CC}\end{array}$ & $\begin{array}{l}65.92 \\
70.52 \\
\end{array}$ & $\begin{array}{l}7, C C \\
8, C C \\
\end{array}$ & $\begin{array}{l}61.26 \\
70.42 \\
\end{array}$ & & \\
\hline & & & $\begin{array}{l}\text { D. petterssoni } \\
\rightarrow \text { D. hughesi }\end{array}$ & $\begin{array}{l}8, \mathrm{CC} \\
9-4 \\
\end{array}$ & $\begin{array}{l}70.52 \\
75.42 \\
\end{array}$ & $\begin{array}{l}7, \mathrm{CC} \\
9, \mathrm{CC} \\
\end{array}$ & $\begin{array}{l}61.26 \\
79.87 \\
\end{array}$ & & \\
\hline \multirow[t]{10}{*}{ D. petterssoni } & 11.5 & B & $\begin{array}{l}\text { Diartus } \\
\text { hughesi }\end{array}$ & $\begin{array}{l}8, \mathrm{CC} \\
9-4 \\
\end{array}$ & $\begin{array}{l}70.52 \\
75.42 \\
\end{array}$ & $\begin{array}{l}8, C C \\
9, C C \\
\end{array}$ & $\begin{array}{l}70.42 \\
79.87 \\
\end{array}$ & & \\
\hline & 11.6 & $\mathrm{~T}$ & $\begin{array}{c}\text { Stichocorys } \\
\text { wolffii }\end{array}$ & $\begin{array}{l}8, \mathrm{CC} \\
9-4 \\
\end{array}$ & $\begin{array}{l}70.52 \\
75.42 \\
\end{array}$ & $\begin{array}{l}8, C C \\
9, C C \\
\end{array}$ & $\begin{array}{l}70.42 \\
79.87 \\
\end{array}$ & & \\
\hline & & $\mathbf{T}$ & $\begin{array}{c}\text { Cyrtocapsella } \\
\text { japonica }\end{array}$ & $\begin{array}{l}8, \mathrm{CC} \\
9-4 \\
\end{array}$ & $\begin{array}{l}70.52 \\
75.42 \\
\end{array}$ & $\begin{array}{l}8, C C \\
9, C C \\
\end{array}$ & $\begin{array}{l}70.42 \\
79.87 \\
\end{array}$ & & \\
\hline & & B & $\begin{array}{c}\begin{array}{c}\text { Phormostichoartus } \\
\text { doliolum }\end{array} \\
\end{array}$ & $\begin{array}{l}9-4 \\
9, \mathrm{CC}\end{array}$ & $\begin{array}{l}75.42 \\
79.96 \\
\end{array}$ & $\begin{array}{l}8, C C \\
9, C C \\
\end{array}$ & $\begin{array}{l}70.42 \\
79.87 \\
\end{array}$ & & \\
\hline & & $\mathbf{T}$ & $\begin{array}{l}\text { Lithopera } \\
\text { thornburgi }\end{array}$ & $\begin{array}{l}9, \mathrm{CC} \\
10-4 \\
\end{array}$ & $\begin{array}{l}79.96 \\
84.92 \\
\end{array}$ & $\begin{array}{l}8, C C \\
9, C C \\
\end{array}$ & $\begin{array}{l}70.42 \\
79.87 \\
\end{array}$ & & \\
\hline & & $\mathbf{T}$ & $\begin{array}{r}\text { Spirocyrtis } \\
\text { subtilis }\end{array}$ & $\begin{array}{l}9, \mathrm{CC} \\
10-4 \\
\end{array}$ & $\begin{array}{l}79.96 \\
84.92 \\
\end{array}$ & $\begin{array}{l}8, C C \\
9, C C \\
\end{array}$ & $\begin{array}{l}70.42 \\
79.87 \\
\end{array}$ & & \\
\hline & & B & $\begin{array}{l}\text { Didymocyrtis } \\
\text { antepenultima }\end{array}$ & $\begin{array}{l}9, \mathrm{CC} \\
10-4 \\
\end{array}$ & $\begin{array}{l}79.96 \\
84.92 \\
\end{array}$ & $\begin{array}{l}8, C C \\
9, C C \\
\end{array}$ & $\begin{array}{l}70.42 \\
79.87 \\
\end{array}$ & & \\
\hline & & B & $\begin{array}{r}\text { Lithopera } \\
\text { bacca }\end{array}$ & $\begin{array}{l}9, \mathrm{CC} \\
10-4 \\
\end{array}$ & $\begin{array}{l}79.96 \\
84.92 \\
\end{array}$ & $\begin{array}{l}9, \mathrm{CC} \\
10, \mathrm{C} \\
\end{array}$ & $\begin{array}{l}79.87 \\
87.19 \\
\end{array}$ & & \\
\hline & & $\mathrm{T}$ & $\begin{array}{r}\text { Carpocanopsis } \\
\text { cristata s.s. }\end{array}$ & {$\left[\begin{array}{l}9-4 \\
9, \mathrm{CC}\end{array}\right.$} & $\left.\begin{array}{l}70.50 \\
79.96\end{array}\right]$ & $\begin{array}{l}9, \mathrm{CC} \\
10, \mathrm{CC} \\
\end{array}$ & $\begin{array}{l}79.87 \\
87.19 \\
\end{array}$ & & \\
\hline & & B & $\begin{array}{c}\text { Botryostrobus } \\
\text { hramlettei }\end{array}$ & $\begin{array}{l}12-4 \\
12, \mathrm{CC}\end{array}$ & $\begin{array}{l}103.52 \\
108.14\end{array}$ & $\begin{array}{l}11, \mathrm{CC} \\
12, \mathrm{CC}\end{array}$ & $\begin{array}{r}98.91 \\
108.17 \\
\end{array}$ & & \\
\hline
\end{tabular}




\section{A. NIGRINI}

Table 3. (Continued).

\begin{tabular}{|c|c|c|c|c|c|c|c|c|c|}
\hline \multirow[b]{2}{*}{ Zone } & \multirow[b]{2}{*}{$\begin{array}{l}\text { Age } \\
(\mathrm{Ma})\end{array}$} & \multirow[b]{2}{*}{$\begin{array}{c}\text { Top } \\
\text { or } \\
\text { bottom }\end{array}$} & \multirow[b]{2}{*}{ Species } & \multicolumn{2}{|c|}{ Hole 574} & \multicolumn{2}{|c|}{ Hole 574A } & \multicolumn{2}{|c|}{ Hole 574B } \\
\hline & & & & Core-Section & $\begin{array}{l}\text { Sub-bottom } \\
\text { depth } \\
\text { (m) }\end{array}$ & Core-Section & $\begin{array}{l}\text { Sub-bottom } \\
\text { depth } \\
\text { (m) }\end{array}$ & Core-Section & $\begin{array}{l}\text { Sub-bottom } \\
\text { depth } \\
\text { (m) }\end{array}$ \\
\hline \multirow[t]{8}{*}{ D. petterssoni } & 11.8 & $\mathbf{T}$ & $\begin{array}{c}\text { Cyrtocapsella } \\
\text { cornuta }\end{array}$ & $\begin{array}{l}12-4^{2} \\
12, \mathrm{CC}\end{array}$ & $\begin{array}{l}103.52 \\
108.14\end{array}$ & $\begin{array}{l}12, \mathrm{CC} \\
13, \mathrm{CC}\end{array}$ & $\begin{array}{l}108.17 \\
113.08\end{array}$ & & \\
\hline & {$[12.5]$} & $\mathrm{T}$ & $\begin{array}{c}\text { Cyrtocapsella } \\
\text { tetrapera }\end{array}$ & $\begin{array}{l}12-4 \\
12, \mathrm{CC}\end{array}$ & $\begin{array}{l}103.52 \\
108.14\end{array}$ & $\begin{array}{l}12, \mathrm{CC} \\
13, \mathrm{CC}\end{array}$ & $\begin{array}{l}108.17 \\
113.08\end{array}$ & & \\
\hline & & $\mathrm{T}$ & $\begin{array}{r}\text { Lithopera } \\
\text { renzae }\end{array}$ & $\begin{array}{l}12-4 \\
12, \mathrm{CC} \\
\end{array}$ & $\begin{array}{l}103.02 \\
108.14 \\
\end{array}$ & $\begin{array}{l}12, \mathrm{CC} \\
13, \mathrm{CC} \\
\end{array}$ & $\begin{array}{l}108.17 \\
113.08 \\
\end{array}$ & & \\
\hline & 11.8 & $\mathbf{T}$ & $\begin{array}{l}\text { Dorcadospyris } \\
\text { alata }\end{array}$ & $\begin{array}{l}12-4 \\
12, \mathrm{CC}\end{array}$ & $\begin{array}{l}103.52 \\
108.14 \\
\end{array}$ & $\begin{array}{l}12, \mathrm{CC} \\
13, \mathrm{CC}\end{array}$ & $\begin{array}{l}108.17 \\
113.08 \\
\end{array}$ & & \\
\hline & & $\mathbf{T}$ & $\begin{array}{c}\text { Giraffospyris } \\
\text { toxaria }\end{array}$ & $\begin{array}{l}13-4 \\
13, \mathrm{CC}\end{array}$ & $\begin{array}{l}113.02 \\
117.65 \\
\end{array}$ & $\begin{array}{l}13, \mathrm{CC} \\
14, \mathrm{CC}\end{array}$ & $\begin{array}{l}113.08 \\
118.03 \\
\end{array}$ & & \\
\hline & & B & $\begin{array}{c}\text { Cyrtocapsella } \\
\text { japonica }\end{array}$ & $\begin{array}{l}14, \mathrm{CC} \\
15, \mathrm{CC}\end{array}$ & $\begin{array}{l}122.53 \\
128.73\end{array}$ & {$\left[\begin{array}{l}12, \mathrm{CC} \\
13, \mathrm{CC}\end{array}\right.$} & $\left.\begin{array}{l}108.17 \\
113.08\end{array}\right]$ & & \\
\hline & & $\mathrm{T}$ & $\begin{array}{c}\text { Didymocyrtis } \\
\text { mammifera }\end{array}$ & $\begin{array}{l}14, \mathrm{CC} \\
15, \mathrm{CC} \\
\end{array}$ & $\begin{array}{l}122.53 \\
128.73\end{array}$ & $\begin{array}{l}15, \mathrm{CC} \\
16, \mathrm{CC}\end{array}$ & $\begin{array}{l}122.68 \\
127.08 \\
\end{array}$ & & \\
\hline & 12.3 & B & $\begin{array}{l}\text { Diartus } \\
\quad \text { petterssoni }\end{array}$ & $\begin{array}{l}14, \mathrm{CC} \\
15, \mathrm{CC} \\
\end{array}$ & $\begin{array}{l}122.53 \\
128.73\end{array}$ & $\begin{array}{l}15, \mathrm{CC} \\
16, \mathrm{CC}\end{array}$ & $\begin{array}{l}122.68 \\
127.08 \\
\end{array}$ & & \\
\hline \multirow[t]{17}{*}{ D. alata } & 14.2 & B & $\begin{array}{l}\text { Lithopera } \\
\text { neotera }\end{array}$ & $\begin{array}{l}16, \mathrm{CC} \\
17, \mathrm{CC}\end{array}$ & $\begin{array}{l}132.56 \\
137.57\end{array}$ & $\begin{array}{l}16, \mathrm{CC} \\
17, \mathrm{CC}\end{array}$ & $\begin{array}{l}127.08 \\
132.66\end{array}$ & & \\
\hline & & B & $\begin{array}{l}\text { Lithopera } \\
\text { thornburgi }\end{array}$ & $\begin{array}{l}16, \mathrm{CC} \\
17, \mathrm{CC} \\
\end{array}$ & $\begin{array}{l}132.56 \\
137.57 \\
\end{array}$ & $\begin{array}{l}16, \mathrm{CC} \\
17, \mathrm{CC} \\
\end{array}$ & $\begin{array}{l}127.08 \\
132.66 \\
\end{array}$ & & \\
\hline & & B & $\begin{array}{l}\text { Phormostichoartus } \\
\text { corbula }\end{array}$ & $\begin{array}{l}17, \mathrm{CC} \\
18, \mathrm{CC} \\
\end{array}$ & $\begin{array}{l}137.57 \\
142.18 \\
\end{array}$ & {$\left[\begin{array}{l}15, \mathrm{CC} \\
16, \mathrm{CC}\end{array}\right.$} & $\left.\begin{array}{l}122.68 \\
127.08\end{array}\right]$ & & \\
\hline & & B & $\begin{array}{c}\text { Dictyocoryne } \\
\text { ontongensis }\end{array}$ & $\begin{array}{l}18, \mathrm{CC} \\
19, \mathrm{CC} \\
\end{array}$ & $\begin{array}{l}142.18 \\
148.24 \\
\end{array}$ & $\begin{array}{l}17, \mathrm{CC} \\
18, \mathrm{CC}\end{array}$ & $\begin{array}{l}132.66 \\
141.43 \\
\end{array}$ & & \\
\hline & {$[13.8]$} & B & $\begin{array}{c}\text { Didymocyrtis } \\
\text { laticonus }\end{array}$ & $\begin{array}{l}18, \mathrm{CC} \\
19, \mathrm{CC} \\
\end{array}$ & $\begin{array}{l}142.18 \\
148.24 \\
\end{array}$ & $\begin{array}{l}18, \mathrm{CC} \\
19, \mathrm{CC} \\
\end{array}$ & $\begin{array}{l}141.43 \\
150.90 \\
\end{array}$ & & \\
\hline & & $\mathbf{T}$ & $\begin{array}{c}\text { Liriospyris } \\
\text { parkerae }\end{array}$ & $\begin{array}{l}20, \mathrm{CC} \\
21, \mathrm{CC}\end{array}$ & $\begin{array}{l}152.48 \\
157.52 \\
\end{array}$ & $\begin{array}{l}19, \mathrm{CC} \\
20, \mathrm{CC}\end{array}$ & $\begin{array}{l}150.90 \\
160.17\end{array}$ & & \\
\hline & 14.75 & $\mathbf{T}$ & $\begin{array}{c}\text { Calocycletta } \\
\text { virginis }\end{array}$ & $\begin{array}{l}20, C C \\
21, C C \\
\end{array}$ & $\begin{array}{l}152.48 \\
157.52 \\
\end{array}$ & {$\left[\begin{array}{l}20, \mathrm{CC} \\
21, \mathrm{CC}\end{array}\right.$} & $\left.\begin{array}{l}160.17 \\
167.38\end{array}\right]$ & & \\
\hline & & $\mathbf{T}$ & $\begin{array}{c}\text { Carpocanopsis } \\
\text { bramlettei }\end{array}$ & $\begin{array}{l}20, \mathrm{CC} \\
21, \mathrm{CC} \\
\end{array}$ & $\begin{array}{l}152.48 \\
157.52 \\
\end{array}$ & $\begin{array}{l}19, \mathrm{CC} \\
20, \mathrm{CC} \\
\end{array}$ & $\begin{array}{l}150.90 \\
160.17 \\
\end{array}$ & & \\
\hline & & $\mathbf{T}$ & $\begin{array}{c}\text { Acrocubus } \\
\text { octopylus }\end{array}$ & {$\left[\begin{array}{l}18, \mathrm{CC} \\
19, \mathrm{CC}\end{array}\right.$} & $\left.\begin{array}{l}142.18 \\
148.24\end{array}\right]$ & $\begin{array}{l}19, \mathrm{CC} \\
20, \mathrm{CC} \\
\end{array}$ & $\begin{array}{l}150.90 \\
160.17 \\
\end{array}$ & & \\
\hline & 15.35 & $\mathbf{T}$ & $\begin{array}{c}\text { Calocycletta } \\
\text { costata }\end{array}$ & $\begin{array}{l}21, \mathrm{CC} \\
22, \mathrm{CC} \\
\end{array}$ & $\begin{array}{l}157.52 \\
162.55 \\
\end{array}$ & $\begin{array}{l}20, C C \\
21, C C \\
\end{array}$ & $\begin{array}{l}160.17 \\
167.17 \\
\end{array}$ & & \\
\hline & & $\mathbf{T}$ & $\begin{array}{c}\text { Didymocyrtis } \\
\text { tubaria }\end{array}$ & $\begin{array}{l}21, \mathrm{CC} \\
22, \mathrm{CC} \\
\end{array}$ & $\begin{array}{l}157.52 \\
162.55 \\
\end{array}$ & $\begin{array}{l}20, \mathrm{CC} \\
21, \mathrm{CC}\end{array}$ & $\begin{array}{l}160.17 \\
167.17 \\
\end{array}$ & & \\
\hline & & T & $\begin{array}{c}\text { Didymocyrtis } \\
\text { violina }\end{array}$ & $\begin{array}{l}21, \mathrm{CC} \\
22, \mathrm{CC}\end{array}$ & $\begin{array}{l}157.52 \\
162.55 \\
\end{array}$ & $\begin{array}{l}20, \mathrm{CC} \\
21, \mathrm{CC} \\
\end{array}$ & $\begin{array}{l}160.17 \\
167.17 \\
\end{array}$ & & \\
\hline & 15.8 & $\mathbf{T}$ & $\begin{array}{c}\text { Dorcadospyris } \\
\text { dentata }\end{array}$ & $\begin{array}{l}21, \mathrm{CC} \\
22, \mathrm{CC}\end{array}$ & $\begin{array}{l}157.52 \\
162.55 \\
\end{array}$ & $\begin{array}{l}20, \mathrm{CC} \\
21, \mathrm{CC} \\
\end{array}$ & $\begin{array}{l}160.17 \\
167.17 \\
\end{array}$ & & \\
\hline & & $\mathrm{T}$ & $\begin{array}{l}\text { Liriospyris } \\
\text { stauropora }\end{array}$ & $\begin{array}{l}25, \mathrm{CC} \\
26, \mathrm{CC} \\
\end{array}$ & $\begin{array}{l}177.79 \\
182.19 \\
\end{array}$ & $\begin{array}{l}20, \mathrm{CC} \\
21, \mathrm{CC} \\
\end{array}$ & $\begin{array}{l}160.17 \\
167.17 \\
\end{array}$ & & \\
\hline & & & $\begin{array}{l}\text { L. stauropora } \\
\rightarrow \text { L. parkeri }\end{array}$ & $\begin{array}{l}25, \mathrm{CC} \\
29, \mathrm{CC} \\
\end{array}$ & $\begin{array}{l}177.79 \\
197.18 \\
\end{array}$ & $\begin{array}{l}20, \mathrm{CC} \\
23, \mathrm{CC}\end{array}$ & $\begin{array}{l}160.17 \\
186.23 \\
\end{array}$ & & \\
\hline & & $\mathbf{T}$ & $\begin{array}{c}\text { Dorcadospyris } \\
\text { forcipata }\end{array}$ & $\begin{array}{l}25, \mathrm{CC} \\
26, \mathrm{CC} \\
\end{array}$ & $\begin{array}{l}177.79 \\
182.19 \\
\end{array}$ & $\begin{array}{l}20, \mathrm{CC} \\
21, \mathrm{CC} \\
\end{array}$ & $\begin{array}{l}160.17 \\
167.17 \\
\end{array}$ & & \\
\hline & & & $\begin{array}{l}\text { D. dentata } \\
\rightarrow \text { D. alata }\end{array}$ & $\begin{array}{l}25, \mathrm{CC} \\
26, \mathrm{CC} \\
\end{array}$ & $\begin{array}{l}177.79 \\
182.19 \\
\end{array}$ & $\begin{array}{l}20, \mathrm{CC} \\
21, \mathrm{CC}\end{array}$ & $\begin{array}{l}160.17 \\
167.17 \\
\end{array}$ & & \\
\hline \multirow[t]{3}{*}{ C. costata } & 15.7 & B & $\begin{array}{l}\text { Dorcadospyris } \\
\quad \text { alata }\end{array}$ & $\begin{array}{l}27, \mathrm{CC} \\
28, \mathrm{CC} \\
\end{array}$ & $\begin{array}{l}187.14 \\
192.27 \\
\end{array}$ & $\begin{array}{l}21, \mathrm{CC} \\
22, \mathrm{CC} \\
\end{array}$ & $\begin{array}{l}167.17 \\
178.00 \\
\end{array}$ & & \\
\hline & & $\mathbf{T}$ & $\begin{array}{c}\text { Eucyrtidium } \\
\text { diaphanes }\end{array}$ & {$\left[\begin{array}{l}26, \mathrm{CC} \\
27, \mathrm{CC}\end{array}\right.$} & $\left.\begin{array}{l}182.19 \\
187.14\end{array}\right]$ & $\begin{array}{l}22, \mathrm{CC} \\
23, \mathrm{CC} \\
\end{array}$ & $\begin{array}{l}178.00 \\
186.23 \\
\end{array}$ & & \\
\hline & & B & $\begin{array}{c}\text { Liriospyris } \\
\text { parkerae }\end{array}$ & $\begin{array}{l}28, C C \\
29, C C \\
\end{array}$ & $\begin{array}{l}192.27 \\
197.18 \\
\end{array}$ & $\begin{array}{l}22, \mathrm{CC} \\
23, \mathrm{CC} \\
\end{array}$ & $\begin{array}{l}178.00 \\
186.23 \\
\end{array}$ & & \\
\hline
\end{tabular}


Table 3. (Continued).

\begin{tabular}{|c|c|c|c|c|c|c|c|c|c|}
\hline \multirow[b]{2}{*}{ Zone } & \multirow[b]{2}{*}{$\begin{array}{l}\mathrm{Age} \\
\text { (Ma) }\end{array}$} & \multirow[b]{2}{*}{$\begin{array}{l}\text { Top } \\
\text { or } \\
\text { bottom }\end{array}$} & \multirow[b]{2}{*}{ Species } & \multicolumn{2}{|c|}{ Hole 574} & \multicolumn{2}{|c|}{ Hole 574A } & \multicolumn{2}{|c|}{ Hole 574B } \\
\hline & & & & Core-Section & $\begin{array}{l}\text { Sub-bottom } \\
\text { depth } \\
\text { (m) }\end{array}$ & Core-Section & $\begin{array}{l}\text { Sub-bottom } \\
\text { depth } \\
\text { (m) }\end{array}$ & Core-Section & $\begin{array}{l}\text { Sub-bottom } \\
\text { depth } \\
\text { (m) }\end{array}$ \\
\hline \multirow[t]{10}{*}{ C. costata } & \multirow{7}{*}{16.2} & $\mathrm{~T}$ & $\begin{array}{c}\text { Carpocanopsis } \\
\text { cingulata }\end{array}$ & $\begin{array}{l}28, C C \\
29, C C\end{array}$ & $\begin{array}{l}192.27 \\
197.18\end{array}$ & & & & \\
\hline & & $\mathrm{T}$ & $\begin{array}{r}\text { Didymocyrtis } \\
\text { prismatica }\end{array}$ & {$\left[\begin{array}{l}26, \mathrm{CC} \\
27, \mathrm{CC}\end{array}\right.$} & $\left.\begin{array}{l}182.19 \\
187.14\end{array}\right]$ & & & $\begin{array}{l}1-2 \\
1, \mathrm{CC}\end{array}$ & $\begin{array}{l}196.42 \\
202.21\end{array}$ \\
\hline & & B & $\begin{array}{r}\text { Carpocanopsis } \\
\text { cristata s.s. }\end{array}$ & $\begin{array}{l}28, \mathrm{CC} \\
29, \mathrm{CC}\end{array}$ & $\begin{array}{l}192.27 \\
197.18\end{array}$ & & & $\begin{array}{l}1-2 \\
1, \mathrm{CC}\end{array}$ & $\begin{array}{l}196.42 \\
202.21\end{array}$ \\
\hline & & $\mathrm{T}$ & $\begin{array}{l}\text { Carpocanopsis } \\
\text { favosa }\end{array}$ & $\begin{array}{l}28, \mathrm{CC} \\
29, \mathrm{CC}\end{array}$ & $\begin{array}{l}192.27 \\
197.18\end{array}$ & & & $\begin{array}{l}1-2 \\
1, \mathrm{CC}\end{array}$ & $\begin{array}{l}196.42 \\
202.21 \\
\end{array}$ \\
\hline & & B & $\begin{array}{r}\text { Lithopera } \\
\text { renzae }\end{array}$ & $\begin{array}{l}28, C C \\
29, C C\end{array}$ & $\begin{array}{l}192.27 \\
197.18\end{array}$ & & & $\begin{array}{l}1-2 \\
1, \mathrm{CC}\end{array}$ & $\begin{array}{l}196.42 \\
202.21\end{array}$ \\
\hline & & B & $\begin{array}{l}\text { Acrocubus } \\
\text { octopylus }\end{array}$ & $\begin{array}{l}29, \mathrm{CC} \\
30, \mathrm{CC}\end{array}$ & $\begin{array}{l}197.18 \\
202.16\end{array}$ & & & $\begin{array}{l}1-2 \\
1, \mathrm{CC}\end{array}$ & $\begin{array}{l}196.42 \\
202.21\end{array}$ \\
\hline & & B & $\begin{array}{c}\text { Giraffospyris } \\
\text { toxaria }\end{array}$ & $\begin{array}{l}29, \mathrm{CC} \\
30, \mathrm{CC}\end{array}$ & $\begin{array}{l}197.18 \\
202.16\end{array}$ & & & $\begin{array}{l}1-2 \\
1, \mathrm{CC}\end{array}$ & $\begin{array}{l}196.42 \\
202.21\end{array}$ \\
\hline & \multirow[t]{2}{*}{16.5} & $\mathrm{~T}$ & $\begin{array}{c}\text { Lychnocanoma } \\
\text { elongata }\end{array}$ & & & & & $\begin{array}{l}2, \mathrm{CC} \\
3, \mathrm{CC}\end{array}$ & $\begin{array}{l}213.38 \\
218.78\end{array}$ \\
\hline & & B & $\begin{array}{l}\text { Didymocyrtis } \\
\text { mammifera }\end{array}$ & & & & & $\begin{array}{l}2, \mathrm{CC} \\
3, \mathrm{CC}\end{array}$ & $\begin{array}{l}213.38 \\
218.78\end{array}$ \\
\hline & 17.3 & B & $\begin{array}{c}\text { Calocycletta } \\
\text { costata }\end{array}$ & & & & & $\begin{array}{l}2, \mathrm{CC} \\
3, \mathrm{CC}\end{array}$ & $\begin{array}{l}213.38 \\
218.78\end{array}$ \\
\hline \multirow[t]{5}{*}{ S. wolffii } & \multirow[t]{3}{*}{17.1} & B & $\begin{array}{c}\text { Dorcadospyris } \\
\text { dentata }\end{array}$ & & & & & $\begin{array}{l}2, \mathrm{CC} \\
3, \mathrm{CC}\end{array}$ & $\begin{array}{l}213.38 \\
218.78\end{array}$ \\
\hline & & B & $\begin{array}{c}\text { Calocycletta } \\
\text { caepa }\end{array}$ & & & & & $\begin{array}{l}3, \mathrm{CC} \\
4, \mathrm{CC}\end{array}$ & $\begin{array}{l}218.78 \\
226.67\end{array}$ \\
\hline & & B & $\begin{array}{l}\text { Liriospyris } \\
\text { stauropora }\end{array}$ & & : & & & $\begin{array}{l}4, \mathrm{CC} \\
5, \mathrm{CC}\end{array}$ & $\begin{array}{l}226.67 \\
238.79 \\
\end{array}$ \\
\hline & {$[20.4]$} & $\mathrm{T}$ & $\begin{array}{c}\text { Dorcadospyris } \\
\text { ateuchus }\end{array}$ & & & & & $\begin{array}{l}4, C C \\
5, C C\end{array}$ & $\begin{array}{l}226.67 \\
238.79\end{array}$ \\
\hline & 17.6 & B & $\begin{array}{c}\text { Stichocorys } \\
\text { wolffii }\end{array}$ & & & & & $\begin{array}{l}4, \mathrm{CC} \\
5, \mathrm{CC} \\
\end{array}$ & $\begin{array}{l}226.67 \\
238.79 \\
\end{array}$ \\
\hline \multirow[t]{5}{*}{ S. delmontensis } & & B & $\begin{array}{l}\text { Siphostichartus } \\
\text { corona }\end{array}$ & & & & & $\begin{array}{l}8, \mathrm{CC} \\
9, \mathrm{CC}\end{array}$ & $\begin{array}{l}265.85 \\
275.28 \\
\end{array}$ \\
\hline & \multirow[t]{2}{*}{19.25} & B & $\begin{array}{c}\text { Didymocyrtis } \\
\text { violina }\end{array}$ & & & & & $\begin{array}{l}9, \mathrm{CC} \\
10-3\end{array}$ & $\begin{array}{l}275.28 \\
283.42 \\
\end{array}$ \\
\hline & & B & $\begin{array}{c}\text { Didymocyrtis } \\
\text { tubaria }\end{array}$ & & & & & $\begin{array}{l}9, \mathrm{CC} \\
10-3\end{array}$ & $\begin{array}{l}275.28 \\
283.42 \\
\end{array}$ \\
\hline & \multirow[t]{2}{*}{20.3} & B & $\begin{array}{l}\text { Stichocorys } \\
\text { delmontensis }\end{array}$ & & & & & $\begin{array}{l}9, \mathrm{CC} \\
10, \mathrm{CC}\end{array}$ & $\begin{array}{l}275.28 \\
289.54\end{array}$ \\
\hline & & B & $\begin{array}{c}\text { Carpocanopsis } \\
\text { bramlettei }\end{array}$ & & & & & $\begin{array}{l}9, \mathrm{CC} \\
10, \mathrm{CC}\end{array}$ & $\begin{array}{l}275.28 \\
289.54 \\
\end{array}$ \\
\hline \multirow[t]{8}{*}{ C. tetrapera } & \multirow[t]{2}{*}{21.3} & $\mathbf{T}$ & $\begin{array}{r}\text { Theocyrtis } \\
\text { annosa }\end{array}$ & & & & & $\begin{array}{l}10, \mathrm{CC} \\
11, \mathrm{CC}\end{array}$ & $\begin{array}{l}289.54 \\
294.00\end{array}$ \\
\hline & & $\mathrm{T}$ & $\begin{array}{c}\text { Calocycletta } \\
\text { robusta }\end{array}$ & & & & & $\begin{array}{l}11, \mathrm{CC} \\
12, \mathrm{CC}\end{array}$ & $\begin{array}{l}294.00 \\
308.39\end{array}$ \\
\hline & \multirow[t]{4}{*}{21.35} & B & $\begin{array}{c}\text { Calocycletta } \\
\text { virginis }\end{array}$ & & & & & $\begin{array}{l}12, \mathrm{CC} \\
13, \mathrm{CC}\end{array}$ & $\begin{array}{l}308.39 \\
314.94\end{array}$ \\
\hline & & $\mathbf{T}$ & $\begin{array}{c}\text { Cyclampterium } \\
\text { pegetrum }\end{array}$ & & & & & $\begin{array}{l}13, \mathrm{CC} \\
14, \mathrm{CC} \\
\end{array}$ & $\begin{array}{l}314.94 \\
321.38 \\
\end{array}$ \\
\hline & & B & $\begin{array}{l}\text { Botryastrobus } \\
\text { miralestensis }\end{array}$ & & & & & $\begin{array}{l}13, \mathrm{CC} \\
14, \mathrm{CC}\end{array}$ & $\begin{array}{l}314.94 \\
321.38\end{array}$ \\
\hline & & B & $\begin{array}{c}\text { Carpocanopsis } \\
\text { cingulata }\end{array}$ & & & & & $\begin{array}{l}13, \mathrm{CC} \\
14, \mathrm{CC} \\
\end{array}$ & $\begin{array}{l}314.94 \\
321.38 \\
\end{array}$ \\
\hline & 22.2 & B & $\begin{array}{c}\text { Cyrtocapsella } \\
\text { cornuta }\end{array}$ & & & & & $\begin{array}{l}14, C C \\
15-3\end{array}$ & $\begin{array}{l}321.38 \\
330.92 \\
\end{array}$ \\
\hline & 22.2 & B & $\begin{array}{c}\text { Cyrtocapsella } \\
\text { tetrapera }\end{array}$ & & & & & $\begin{array}{l}14, \mathrm{CC} \\
15-3\end{array}$ & $\begin{array}{l}321.38 \\
330.92\end{array}$ \\
\hline
\end{tabular}


Table 3. (Continued).

\begin{tabular}{|c|c|c|c|c|c|c|c|c|c|}
\hline \multirow[b]{2}{*}{ Zone } & \multirow[b]{2}{*}{$\begin{array}{l}\text { Age } \\
\text { (Ma) }\end{array}$} & \multirow[b]{2}{*}{$\begin{array}{c}\text { Top } \\
\text { or } \\
\text { bottom }\end{array}$} & \multirow[b]{2}{*}{ Species } & \multicolumn{2}{|c|}{ Hole 574} & \multicolumn{2}{|c|}{ Hole $574 \mathrm{~A}$} & \multicolumn{2}{|c|}{ Hole 574B } \\
\hline & & & & Core-Section & $\begin{array}{l}\text { Sub-bottom } \\
\text { depth } \\
\text { (m) }\end{array}$ & Core-Section & $\begin{array}{l}\text { Sub-bottom } \\
\text { depth } \\
\text { (m) }\end{array}$ & Core-Section & $\begin{array}{l}\text { Sub-bottom } \\
\text { depth } \\
\text { (m) }\end{array}$ \\
\hline \multirow[t]{3}{*}{ L. elongata } & & B & $\begin{array}{c}\text { Carpocanopsis } \\
\text { favosa }\end{array}$ & & & & & $\begin{array}{l}14, \mathrm{CC} \\
15-3\end{array}$ & $\begin{array}{l}321.38 \\
330.92 \\
\end{array}$ \\
\hline & 22.5 & $\mathbf{T}$ & $\begin{array}{c}\text { Artophormis } \\
\text { gracilis }\end{array}$ & & & & & $\begin{array}{l}15-3 \\
15, \mathrm{CC}\end{array}$ & $\begin{array}{l}330.92 \\
336.85\end{array}$ \\
\hline & 25.9 & B & $\begin{array}{c}\text { Lychnocanoma } \\
\text { elongata }\end{array}$ & & & & & $\begin{array}{l}18, \mathrm{CC} \\
19, \mathrm{CC}\end{array}$ & $\begin{array}{l}358.48 \\
368.17\end{array}$ \\
\hline \multirow[t]{9}{*}{ D. ateuchus } & & B & $\begin{array}{c}\text { Calocycletta } \\
\text { robusta }\end{array}$ & & & & & $\begin{array}{l}18, \mathrm{CC} \\
19, \mathrm{CC} \\
\end{array}$ & $\begin{array}{l}358.48 \\
368.17 \\
\end{array}$ \\
\hline & & B & $\begin{array}{c}\text { Dorcadospyris } \\
\text { forcipata }\end{array}$ & & & & & $\begin{array}{l}20, \mathrm{CC} \\
21, \mathrm{CC}\end{array}$ & $\begin{array}{l}384.25 \\
390.65\end{array}$ \\
\hline & & $T$ & $\begin{array}{r}\text { Lithocyclia } \\
\text { angusta }\end{array}$ & & & & & $\begin{array}{l}24, \mathrm{CC} \\
25, \mathrm{CC}\end{array}$ & $\begin{array}{l}419.47 \\
424.51\end{array}$ \\
\hline & & B & $\begin{array}{r}\text { Theocyrtis } \\
\text { annosa }\end{array}$ & & & & & $\begin{array}{l}25, \mathrm{CC} \\
26, \mathrm{CC}\end{array}$ & $\begin{array}{l}424.51 \\
435.90 \\
\end{array}$ \\
\hline & & $\mathrm{T}$ & $\begin{array}{l}\text { Lithocyclia } \\
\text { crux }\end{array}$ & & & & & $\begin{array}{l}25, \mathrm{CC} \\
26, \mathrm{CC}\end{array}$ & $\begin{array}{l}424.51 \\
435.90\end{array}$ \\
\hline & & B & $\begin{array}{c}\text { Didymocyrtis } \\
\text { prismatica }\end{array}$ & & & & & $\begin{array}{l}25, C C \\
26, C C\end{array}$ & $\begin{array}{l}424.51 \\
435.90\end{array}$ \\
\hline & & $\mathbf{T}$ & $\begin{array}{c}\text { Cyclampterium } \\
\text { milowi }\end{array}$ & & & & & $\begin{array}{l}26, \mathrm{CC} \\
27, \mathrm{CC}\end{array}$ & $\begin{array}{l}435.90 \\
446.86 \\
\end{array}$ \\
\hline & & $\mathrm{T}$ & $\begin{array}{c}\text { Tristylospyris } \\
\text { triceros }\end{array}$ & & & & & $\begin{array}{l}26, \mathrm{CC} \\
27, \mathrm{CC}\end{array}$ & $\begin{array}{l}435.90 \\
446.86\end{array}$ \\
\hline & & & $\begin{array}{l}\text { T. triceros } \\
\rightarrow \text { D. ateuchus }\end{array}$ & & & & & $\begin{array}{l}26, \mathrm{CC} \\
27, \mathrm{CC}\end{array}$ & $\begin{array}{l}435.90 \\
446.86 \\
\end{array}$ \\
\hline \multirow[t]{8}{*}{ T. tuberosa } & 33.0 & $B$ & $\begin{array}{c}\text { Dorcadospyris } \\
\text { ateuchus }\end{array}$ & & & & & $\begin{array}{l}26, \mathrm{CC} \\
27, \mathrm{CC}\end{array}$ & $\begin{array}{l}435.90 \\
446.86 \\
\end{array}$ \\
\hline & & B & $\begin{array}{c}\text { Cyclampterium } \\
\text { pegetrum }\end{array}$ & & & & & $\begin{array}{l}27, \mathrm{CC} \\
28, \mathrm{CC}\end{array}$ & $\begin{array}{l}446.86 \\
451.57 \\
\end{array}$ \\
\hline & & $T$ & $\begin{array}{l}\text { Theocyrtis } \\
\text { tuberosa }\end{array}$ & & & & & $\begin{array}{l}28, C C \\
29, C C \\
\end{array}$ & $\begin{array}{l}451.57 \\
465.27 \\
\end{array}$ \\
\hline & & B & $\begin{array}{l}\text { Eucyrtidium } \\
\text { diaphanes }\end{array}$ & & & & & $\begin{array}{l}28, C C \\
29, C C\end{array}$ & $\begin{array}{l}451.57 \\
465.27\end{array}$ \\
\hline & & B & $\begin{array}{r}\text { Lithocyclia } \\
\text { angusta }\end{array}$ & & & & & $\begin{array}{l}29, \mathrm{CC} \\
30, \mathrm{CC}\end{array}$ & $\begin{array}{l}465.27 \\
474.85\end{array}$ \\
\hline & & B & $\begin{array}{r}\text { Spirocyrtis } \\
\text { subtilis }\end{array}$ & & & & & $\begin{array}{l}31, \mathrm{CC} \\
32, \mathrm{CC}\end{array}$ & $\begin{array}{l}483.15 \\
493.19\end{array}$ \\
\hline & & B & $\begin{array}{l}\text { Cyclampterium } \\
\text { milowi }\end{array}$ & & & & & $\begin{array}{l}33-4 \\
33-5\end{array}$ & $\begin{array}{l}503.50 \\
505.08\end{array}$ \\
\hline & & B & $\begin{array}{l}\text { Theocyrtis } \\
\text { tuberosa }\end{array}$ & & & & & $\begin{array}{l}33-4 \\
33-5\end{array}$ & $\begin{array}{l}503.50 \\
505.08\end{array}$ \\
\hline
\end{tabular}

Note: Pairs of levels and brackets as defined in Table 1 of this appendix.

Table 4. List of radiolarian events for Site 575.

\begin{tabular}{|c|c|c|c|c|c|c|c|c|c|}
\hline \multirow[b]{2}{*}{ Zone } & \multirow[b]{2}{*}{$\begin{array}{l}\text { Age } \\
\text { (Ma) }\end{array}$} & \multirow[b]{2}{*}{$\begin{array}{c}\text { Top } \\
\text { or } \\
\text { bottom }\end{array}$} & \multirow[b]{2}{*}{ Species } & \multicolumn{2}{|c|}{ Hole 575} & \multicolumn{2}{|c|}{ Hole 575A } & \multicolumn{2}{|c|}{ Hole 575B } \\
\hline & & & & Core-Section & $\begin{array}{l}\text { Sub-bottom } \\
\text { depth } \\
\text { (m) }\end{array}$ & Core-Section & $\begin{array}{l}\text { Sub-bottom } \\
\text { depth } \\
\text { (m) }\end{array}$ & Core-Section & $\begin{array}{l}\text { Sub-bottom } \\
\text { depth } \\
\text { (m) }\end{array}$ \\
\hline B. invaginata & 0.3 & B & $\begin{array}{c}\text { Buccinosphaera } \\
\text { invaginata }\end{array}$ & & & & & & \\
\hline \multirow[t]{3}{*}{ C. tuberosa } & 0.44 & $\mathbf{T}$ & $\begin{array}{l}\text { Stylatractus } \\
\text { universus }\end{array}$ & $\begin{array}{l}1-2 \\
1-3\end{array}$ & $\begin{array}{l}1.92 \\
3.42\end{array}$ & & & & \\
\hline & & B & $\begin{array}{c}\text { Collosphaera } \\
\text { tuberosa }\end{array}$ & $\begin{array}{l}1-1 \\
1-2\end{array}$ & $\begin{array}{l}0.42 \\
1.92\end{array}$ & & & & \\
\hline & & B & $\begin{array}{l}\text { Pterocorys } \\
\text { hertwigii }\end{array}$ & $\begin{array}{l}1-2 \\
1-3\end{array}$ & $\begin{array}{l}1.92 \\
3.42\end{array}$ & & & & \\
\hline
\end{tabular}


Table 4. (Continued).

\begin{tabular}{|c|c|c|c|c|c|c|c|c|c|}
\hline \multirow[b]{2}{*}{ Zone } & \multirow[b]{2}{*}{$\begin{array}{l}\text { Age } \\
\text { (Ma) }\end{array}$} & \multirow[b]{2}{*}{$\begin{array}{l}\text { Top } \\
\text { or } \\
\text { bottom }\end{array}$} & \multirow[b]{2}{*}{ Species } & \multicolumn{2}{|c|}{ Hole 575} & \multicolumn{2}{|c|}{ Hole $575 \mathrm{~A}$} & \multicolumn{2}{|c|}{ Hole 575B } \\
\hline & & & & Core-Section & $\begin{array}{l}\text { Sub-bottom } \\
\text { depth } \\
\text { (m) }\end{array}$ & Core-Section & $\begin{array}{l}\text { Sub-bottom } \\
\text { depth } \\
\text { (m) }\end{array}$ & Core-Section & $\begin{array}{l}\text { Sub-bottom } \\
\text { depth } \\
\text { (m) }\end{array}$ \\
\hline A. ypsilon & 1.1 & $T$ & $\begin{array}{c}\text { Anthocyrtidium } \\
\text { angulare }\end{array}$ & & & & & & \\
\hline \multirow[t]{4}{*}{ A. angulare } & & $T$ & $\begin{array}{c}\text { Theocorythium } \\
\text { vetulum }\end{array}$ & {$\left[\begin{array}{l}1-4 \\
1-5\end{array}\right]$} & $\left.\begin{array}{l}4.92 \\
6.42\end{array}\right]$ & & & & \\
\hline & & $\mathrm{T}$ & $\begin{array}{l}\text { Didymocyrtis } \\
\text { avita }\end{array}$ & $\begin{array}{l}1-3 \\
1-4\end{array}$ & $\begin{array}{l}3.42 \\
4.92\end{array}$ & & & $1, \mathrm{CC}$ & $\begin{array}{r}0.00 \\
12.85\end{array}$ \\
\hline & & B & $\begin{array}{c}\text { Lamprocyrtis } \\
\text { nigriniae }\end{array}$ & $\begin{array}{l}1-3 \\
1-4 \\
\end{array}$ & $\begin{array}{l}3.42 \\
4.92 \\
\end{array}$ & & & & \\
\hline & 1.35 & B & $\begin{array}{c}\text { Theocorythium } \\
\text { trachelium }\end{array}$ & $\begin{array}{l}1-3 \\
1-4 \\
\end{array}$ & $\begin{array}{l}3.42 \\
4.92 \\
\end{array}$ & & & & \\
\hline \multirow[t]{2}{*}{ P. prismatium } & 1.5 & $\mathrm{~T}$ & $\begin{array}{l}\text { Pterocanium } \\
\text { prismatium }\end{array}$ & $\begin{array}{l}1-3 \\
1-4 \\
\end{array}$ & $\begin{array}{l}3.42 \\
4.92 \\
\end{array}$ & & & & \\
\hline & & B & $\begin{array}{c}\text { Anthocyrtidium } \\
\text { angulare }\end{array}$ & $\begin{array}{l}1-4 \\
1-5 \\
\end{array}$ & $\begin{array}{l}4.92 \\
6.42 \\
\end{array}$ & & & & \\
\hline \multirow[t]{13}{*}{ S. pentas } & 2.4 & $\mathrm{~T}$ & $\begin{array}{l}\text { Stichocorys } \\
\text { peregrina }\end{array}$ & $\begin{array}{l}1-4 \\
1-5\end{array}$ & $\begin{array}{l}4.92 \\
6.42 \\
\end{array}$ & & & $1, \mathrm{CC}$ & $\begin{array}{r}0.00 \\
12.85 \\
\end{array}$ \\
\hline & & $\mathrm{T}$ & $\begin{array}{l}\text { Phormostichoartus } \\
\quad \text { fistula }\end{array}$ & $\begin{array}{l}1-5 \\
1, \mathrm{CC}\end{array}$ & $\begin{array}{l}6.42 \\
7.27 \\
\end{array}$ & & & $\begin{array}{l}1, \mathrm{CC} \\
2, \mathrm{CC}\end{array}$ & $\begin{array}{l}12.85 \\
21.50 \\
\end{array}$ \\
\hline & 3.4 & $\mathrm{~T}$ & $\begin{array}{c}\text { Spongaster } \\
\text { pentas }\end{array}$ & {$\left[\begin{array}{l}1, \mathrm{CC} \\
2-1\end{array}\right.$} & $\left.\begin{array}{l}7.27 \\
7.30\end{array}\right]$ & & & $1, \mathrm{CC}$ & $\begin{array}{r}0.00 \\
12.85 \\
\end{array}$ \\
\hline & & $\mathrm{T}$ & $\begin{array}{l}\text { Lychnodictyum } \\
\text { audax }\end{array}$ & $\begin{array}{l}1-5 \\
1, \mathrm{CC}\end{array}$ & $\begin{array}{l}6.42 \\
7.27 \\
\end{array}$ & & & $\begin{array}{l}1, \mathrm{CC} \\
2, \mathrm{CC}\end{array}$ & $\begin{array}{l}12.85 \\
21.50 \\
\end{array}$ \\
\hline & & $\mathrm{T}$ & $\begin{array}{l}\text { Phormostichoartus } \\
\text { doliolum }\end{array}$ & $\begin{array}{l}1-5 \\
1, \mathrm{CC}\end{array}$ & $\begin{array}{l}6.42 \\
7.27 \\
\end{array}$ & & & $1, \mathrm{CC}$ & $\begin{array}{r}0.00 \\
12.85 \\
\end{array}$ \\
\hline & & B & $\begin{array}{l}\text { Amphirhopalum } \\
\text { ypsilon }\end{array}$ & $\begin{array}{l}1, \mathrm{CC} \\
2-1\end{array}$ & $\begin{array}{l}7.27 \\
7.30 \\
\end{array}$ & & & $\begin{array}{l}1, \mathrm{CC} \\
2, \mathrm{CC}\end{array}$ & $\begin{array}{r}12.85 \\
21.50 \\
\end{array}$ \\
\hline & & B & $\begin{array}{l}\text { Didymocyrtis } \\
\quad \text { tetrathalamus }\end{array}$ & $\begin{array}{l}1, \mathrm{CC} \\
2-1 \\
\end{array}$ & $\begin{array}{l}7.27 \\
7.30 \\
\end{array}$ & & & & \\
\hline & 3.6 & B & $\begin{array}{c}\text { Spongaster } \\
\text { tetras }\end{array}$ & $\begin{array}{l}1, \mathrm{CC} \\
2-1\end{array}$ & $\begin{array}{l}7.27 \\
7.30 \\
\end{array}$ & & & $1, \mathrm{CC}$ & $\begin{array}{r}0.00 \\
12.85 \\
\end{array}$ \\
\hline & 3.69 & $\mathrm{~T}$ & $\begin{array}{r}\text { Didymocyrtis } \\
\text { penultima } \\
\end{array}$ & $\begin{array}{l}1, \mathrm{CC} \\
2-1\end{array}$ & $\begin{array}{l}7.27 \\
7.30 \\
\end{array}$ & & & $1, \mathrm{CC}$ & $\begin{array}{r}0.00 \\
12.85 \\
\end{array}$ \\
\hline & & $\mathrm{T}$ & $\begin{array}{r}\text { Solenosphaera } \\
\text { omnitubus }\end{array}$ & {$\left[\begin{array}{l}1-5 \\
1, \mathrm{CC}\end{array}\right.$} & $\left.\begin{array}{l}6.42 \\
7.27\end{array}\right]$ & & & $1, \mathrm{CC}$ & $\begin{array}{r}0.00 \\
12.85 \\
\end{array}$ \\
\hline & & B & $\begin{array}{c}\text { Didymocyrtis } \\
\text { avita }\end{array}$ & $\begin{array}{l}1, \mathrm{CC} \\
2-1\end{array}$ & $\begin{array}{l}7.27 \\
7.30 \\
\end{array}$ & & & $\begin{array}{l}1, \mathrm{CC} \\
2, \mathrm{CC}\end{array}$ & $\begin{array}{l}12.85 \\
21.50 \\
\end{array}$ \\
\hline & & $\mathrm{T}$ & $\begin{array}{l}\text { Spongaster } \\
\quad \text { berminghami }\end{array}$ & $\begin{array}{l}2-2 \\
2-4 \\
\end{array}$ & $\begin{array}{r}9.23 \\
12.23 \\
\end{array}$ & & & $\begin{array}{l}1, \mathrm{CC} \\
2, \mathrm{CC} \\
\end{array}$ & $\begin{array}{l}12.85 \\
21.50 \\
\end{array}$ \\
\hline & & & $\begin{array}{l}S . \text { berminghami } \\
\rightarrow S \text {. pentas }\end{array}$ & $\begin{array}{l}2-1 \\
2-4 \\
\end{array}$ & $\begin{array}{r}7.73 \\
12.23 \\
\end{array}$ & & & & \\
\hline \multirow[t]{8}{*}{ S. peregrina } & 4.3 & B & $\begin{array}{c}\text { Spongaster } \\
\text { pentas }\end{array}$ & $\begin{array}{l}2-1 \\
2-2 \\
\end{array}$ & $\begin{array}{l}7.73 \\
9.23 \\
\end{array}$ & & & $\begin{array}{l}1, \mathrm{CC} \\
2, \mathrm{CC}\end{array}$ & $\begin{array}{l}12.85 \\
21.50\end{array}$ \\
\hline & & $\mathrm{T}$ & $\begin{array}{r}\text { Botryostrobus } \\
\text { bramlettei } \\
\end{array}$ & {$\left[\begin{array}{l}1-4 \\
2-1\end{array}\right]$} & $\left.\begin{array}{l}4.92 \\
7.73\end{array}\right]$ & & & $\begin{array}{l}1, \mathrm{CC} \\
2, \mathrm{CC}\end{array}$ & $\begin{array}{l}12.85 \\
21.50\end{array}$ \\
\hline & 4.8 & B & $\begin{array}{l}\text { Pterocanium } \\
\quad \text { prismatium }\end{array}$ & $\begin{array}{l}2-1 \\
2-2\end{array}$ & $\begin{array}{l}7.73 \\
9.23\end{array}$ & & & $\begin{array}{l}1, \mathrm{CC} \\
2, \mathrm{CC}\end{array}$ & $\begin{array}{l}12.85 \\
21.50\end{array}$ \\
\hline & & B & $\begin{array}{l}\text { Botryostrobus } \\
\text { aquilonaris }\end{array}$ & $\begin{array}{l}2-1 \\
2-2 \\
\end{array}$ & $\begin{array}{l}7.73 \\
9.23 \\
\end{array}$ & & & $\begin{array}{l}1, \mathrm{CC} \\
2, \mathrm{CC} \\
\end{array}$ & $\begin{array}{l}12.85 \\
21.50 \\
\end{array}$ \\
\hline & & $\mathbf{T}$ & $\begin{array}{l}\text { Didymocyrtis } \\
\text { antepenultima }\end{array}$ & $\begin{array}{l}1, \mathrm{CC} \\
2-5\end{array}$ & $\begin{array}{r}7.27 \\
13.73 \\
\end{array}$ & & & $\begin{array}{l}1, \mathrm{CC} \\
2, \mathrm{CC}\end{array}$ & $\begin{array}{l}12.85 \\
21.50 \\
\end{array}$ \\
\hline & & $\mathrm{T}$ & $\begin{array}{l}\text { Stichocorys } \\
\quad \text { delmontensis }\end{array}$ & $\begin{array}{l}1, \mathrm{CC} \\
2-4\end{array}$ & $\begin{array}{r}7.27 \\
12.23 \\
\end{array}$ & & & $\begin{array}{l}1, \mathrm{CC} \\
2, \mathrm{CC}\end{array}$ & $\begin{array}{l}12.85 \\
21.50 \\
\end{array}$ \\
\hline & & $\mathrm{T}$ & $\begin{array}{l}\text { Siphostichartus } \\
\text { corona }\end{array}$ & {$\left[\begin{array}{l}1-4 \\
1, \mathrm{CC}\end{array}\right.$} & $\left.\begin{array}{l}4.92 \\
7.27\end{array}\right]$ & & & $\begin{array}{l}1, \mathrm{CC} \\
2, \mathrm{CC}\end{array}$ & $\begin{array}{l}12.85 \\
21.50 \\
\end{array}$ \\
\hline & 5.0 & $\mathrm{~T}$ & $\begin{array}{c}\text { Acrobotrys } \\
\text { tritubus }\end{array}$ & $\begin{array}{l}1-5 \\
2-3\end{array}$ & $\begin{array}{r}6.42 \\
10.73\end{array}$ & & & $1, \mathrm{CC}$ & $\begin{array}{r}0.00 \\
12.85\end{array}$ \\
\hline
\end{tabular}




\section{A. NIGRINI}

Table 4. (Continued).

\begin{tabular}{|c|c|c|c|c|c|c|c|c|c|}
\hline \multirow[b]{2}{*}{ Zone } & \multirow[b]{2}{*}{$\begin{array}{l}\text { Age } \\
(\mathrm{Ma})\end{array}$} & \multirow[b]{2}{*}{$\begin{array}{c}\text { Top } \\
\text { or } \\
\text { bottom }\end{array}$} & \multirow[b]{2}{*}{ Species } & \multicolumn{2}{|c|}{ Hole 575} & \multicolumn{2}{|c|}{ Hole 575A } & \multicolumn{2}{|c|}{ Hole 575B } \\
\hline & & & & Core-Section & $\begin{array}{l}\text { Sub-bottom } \\
\text { depth } \\
\text { (m) }\end{array}$ & Core-Section & $\begin{array}{l}\text { Sub-bottom } \\
\text { depth } \\
\text { (m) }\end{array}$ & Core-Section & $\begin{array}{l}\text { Sub-bottom } \\
\text { depth } \\
\text { (m) }\end{array}$ \\
\hline \multirow[t]{2}{*}{ S. peregrina } & & $\mathrm{T}$ & $\begin{array}{l}\text { Calocycletta } \\
\text { caepa }\end{array}$ & $\begin{array}{l}2-4 \\
2-6\end{array}$ & $\begin{array}{l}12.23 \\
15.23\end{array}$ & & & $1, \mathrm{CC}$ & $\begin{array}{r}0.00 \\
12.85\end{array}$ \\
\hline & & & $\begin{array}{l}\text { S. delmontensis } \\
\rightarrow \text { S. peregrina }\end{array}$ & $\begin{array}{l}2-4 \\
2-6\end{array}$ & $\begin{array}{l}12.23 \\
15.23\end{array}$ & & & $\begin{array}{l}1, \mathrm{CC} \\
2, \mathrm{CC}\end{array}$ & $\begin{array}{l}12.85 \\
21.50\end{array}$ \\
\hline \multirow[t]{3}{*}{ D. penultima } & & $\mathrm{T}$ & $\begin{array}{c}\text { Phormostichoartus } \\
\text { marylandicus }\end{array}$ & $2-4$ & $\begin{array}{l}12.23 \\
16.89\end{array}$ & & & $\begin{array}{l}1, \mathrm{CC} \\
2, \mathrm{CC}\end{array}$ & $\begin{array}{l}12.85 \\
21.50\end{array}$ \\
\hline & 6.55 & B & $\begin{array}{r}\text { Solenosphaera } \\
\text { omnitubus }\end{array}$ & $\begin{array}{l}2, \mathrm{CC} \\
3-3\end{array}$ & $\begin{array}{l}16.89 \\
20.23\end{array}$ & & & $\begin{array}{l}2, \mathrm{CC} \\
3, \mathrm{CC}\end{array}$ & $\begin{array}{l}21.50 \\
29.66\end{array}$ \\
\hline & {$[6.4]$} & B & $\begin{array}{l}\text { Stichocorys } \\
\text { peregrina }\end{array}$ & $\begin{array}{l}2, \mathrm{CC} \\
3-3\end{array}$ & $\begin{array}{l}16.89 \\
20.23\end{array}$ & & & $\begin{array}{l}2, \mathrm{CC} \\
3, \mathrm{CC}\end{array}$ & $\begin{array}{l}21.50 \\
29.66\end{array}$ \\
\hline \multirow[t]{10}{*}{ D. antepenultima } & 8.2 & $\mathrm{~T}$ & $\begin{array}{l}\text { Diartus } \\
\quad \text { hughesi }\end{array}$ & $\begin{array}{l}2, \mathrm{CC} \\
3-3\end{array}$ & $\begin{array}{l}16.89 \\
20.23 \\
\end{array}$ & & & $\begin{array}{l}2, \mathrm{CC} \\
3, \mathrm{CC} \\
\end{array}$ & $\begin{array}{l}21.50 \\
29.66\end{array}$ \\
\hline & & $\mathrm{T}$ & $\begin{array}{l}\text { Lithopera } \\
\text { neotera }\end{array}$ & $\begin{array}{l}2, \mathrm{CC} \\
3-3\end{array}$ & $\begin{array}{l}16.89 \\
20.23\end{array}$ & & & $\begin{array}{l}2, \mathrm{CC} \\
3, \mathrm{CC}\end{array}$ & $\begin{array}{l}21.50 \\
29.66\end{array}$ \\
\hline & & $\mathrm{T}$ & $\begin{array}{l}\text { Dictyocoryne } \\
\text { ontongensis }\end{array}$ & $\begin{array}{l}2, \mathrm{CC} \\
3-3\end{array}$ & $\begin{array}{l}16.89 \\
20.23\end{array}$ & & & $\begin{array}{l}2, \mathrm{CC} \\
3, \mathrm{CC}\end{array}$ & $\begin{array}{l}21.50 \\
29.66\end{array}$ \\
\hline & & $\mathrm{T}$ & $\begin{array}{l}\text { Botryocyrtis } \\
\quad \text { miralestensis }\end{array}$ & $\begin{array}{l}3-3 \\
3, \mathrm{CC}\end{array}$ & $\begin{array}{l}20.23 \\
26.40\end{array}$ & & & $\begin{array}{l}2, \mathrm{CC} \\
3, \mathrm{CC}\end{array}$ & $\begin{array}{l}21.50 \\
29.66\end{array}$ \\
\hline & [11.2] & B & $\begin{array}{c}\text { Acrobotrys } \\
\text { tritubus } \\
\end{array}$ & $\begin{array}{l}3-3 \\
3, \mathrm{CC} \\
\end{array}$ & $\begin{array}{l}20.23 \\
26.40\end{array}$ & & & $\begin{array}{l}2, \mathrm{CC} \\
3, \mathrm{CC} \\
\end{array}$ & $\begin{array}{l}21.50 \\
29.66\end{array}$ \\
\hline & & $\mathrm{T}$ & $\begin{array}{c}\text { Didymocyrtis } \\
\text { laticonus }\end{array}$ & $\begin{array}{l}3-3 \\
3, \mathrm{CC}\end{array}$ & $\begin{array}{l}20.23 \\
26.40\end{array}$ & & & $\begin{array}{l}2, \mathrm{CC} \\
3, \mathrm{CC}\end{array}$ & $\begin{array}{l}21.50 \\
29.66\end{array}$ \\
\hline & 8.2 & B & $\begin{array}{r}\text { Didymocyrtis } \\
\text { penultima }\end{array}$ & $\begin{array}{l}3-3 \\
3, \mathrm{CC}\end{array}$ & $\begin{array}{l}20.23 \\
26.40\end{array}$ & & & $\begin{array}{l}3, \mathrm{CC} \\
4, \mathrm{CC} \\
\end{array}$ & $\begin{array}{l}29.66 \\
39.08 \\
\end{array}$ \\
\hline & 9.7 & $\mathrm{~T}$ & $\begin{array}{l}\text { Diartus } \\
\quad \text { petterssoni }\end{array}$ & $\begin{array}{l}3-3 \\
3, \mathrm{CC}\end{array}$ & $\begin{array}{l}20.23 \\
26.40\end{array}$ & & & $\begin{array}{l}3, \mathrm{CC} \\
4, \mathrm{CC}\end{array}$ & $\begin{array}{l}29.66 \\
39.08\end{array}$ \\
\hline & & B & $\begin{array}{l}\text { Spongaster } \\
\quad \text { berminghami }\end{array}$ & $\begin{array}{l}3-3 \\
3, \mathrm{CC} \\
\end{array}$ & $\begin{array}{l}20.23 \\
26.40 \\
\end{array}$ & & & $\begin{array}{l}3, \mathrm{CC} \\
4, \mathrm{CC} \\
\end{array}$ & $\begin{array}{l}29.66 \\
39.08 \\
\end{array}$ \\
\hline & & & $\begin{array}{l}\text { D. petterssoni } \\
\rightarrow D \text {. hughesi }\end{array}$ & $\begin{array}{l}3, \mathrm{CC} \\
4-4\end{array}$ & $\begin{array}{l}26.40 \\
31.23\end{array}$ & & & $\begin{array}{l}3, \mathrm{CC} \\
4, \mathrm{CC}\end{array}$ & $\begin{array}{l}29.66 \\
39.08\end{array}$ \\
\hline \multirow[t]{13}{*}{ D. petterssoni } & 11.5 & B & $\begin{array}{l}\text { Diartus } \\
\quad \text { hughesi }\end{array}$ & $\begin{array}{l}3, \mathrm{CC} \\
4-4\end{array}$ & $\begin{array}{l}26.40 \\
31.23\end{array}$ & & & $\begin{array}{l}3, \mathrm{CC} \\
4, \mathrm{CC}\end{array}$ & $\begin{array}{l}29.66 \\
39.08\end{array}$ \\
\hline & 11.6 & $\mathrm{~T}$ & $\begin{array}{c}\text { Stichocorys } \\
\text { wolffii }\end{array}$ & $\begin{array}{l}3, \mathrm{CC} \\
4-4\end{array}$ & $\begin{array}{l}26.40 \\
31.23\end{array}$ & & & $\begin{array}{l}3, \mathrm{CC} \\
4, \mathrm{CC}\end{array}$ & $\begin{array}{l}29.66 \\
39.08\end{array}$ \\
\hline & & $\mathrm{T}$ & $\begin{array}{c}\text { Cyrtocapsella } \\
\text { japonica }\end{array}$ & $\begin{array}{l}3, \mathrm{CC} \\
4-4 \\
\end{array}$ & $\begin{array}{l}26.40 \\
31.23 \\
\end{array}$ & & & $\begin{array}{l}3, \mathrm{CC} \\
4, \mathrm{CC}\end{array}$ & $\begin{array}{l}29.66 \\
39.08 \\
\end{array}$ \\
\hline & & B & $\begin{array}{c}\text { Phormostichoartus } \\
\text { doliolum }\end{array}$ & $\begin{array}{l}3, \mathrm{CC} \\
4-4\end{array}$ & $\begin{array}{l}26.40 \\
31.23\end{array}$ & & & $\begin{array}{l}3, \mathrm{CC} \\
4, \mathrm{CC} \\
\end{array}$ & $\begin{array}{l}29.66 \\
39.08 \\
\end{array}$ \\
\hline & & $\mathrm{T}$ & $\begin{array}{l}\text { Lithopera } \\
\text { thornburgi }\end{array}$ & $\begin{array}{l}3, \mathrm{CC} \\
4-4 \\
\end{array}$ & $\begin{array}{l}26.40 \\
31.23 \\
\end{array}$ & & & $\begin{array}{l}3, \mathrm{CC} \\
4, \mathrm{CC} \\
\end{array}$ & $\begin{array}{l}29.66 \\
39.08 \\
\end{array}$ \\
\hline & & $\mathrm{T}$ & $\begin{array}{r}\text { Spirocyrtis } \\
\text { subtilis }\end{array}$ & {$\left[\begin{array}{l}5-3 \\
5, \mathrm{CC}\end{array}\right.$} & $\left.\begin{array}{l}38.43 \\
44.55\end{array}\right]$ & & & {$\left[\begin{array}{l}4, \mathrm{CC} \\
5, \mathrm{CC}\end{array}\right.$} & $\left.\begin{array}{l}39.08 \\
48.28\end{array}\right]$ \\
\hline & & B & $\begin{array}{l}\text { Didymocyrtis } \\
\text { antepenultima }\end{array}$ & $\begin{array}{l}3, \mathrm{CC} \\
4-4 \\
\end{array}$ & $\begin{array}{l}26.40 \\
31.23 \\
\end{array}$ & & & $\begin{array}{l}3, \mathrm{CC} \\
4, \mathrm{CC} \\
\end{array}$ & $\begin{array}{l}29.66 \\
39.08 \\
\end{array}$ \\
\hline & & B & $\begin{array}{r}\text { Lithopera } \\
\text { bacca }\end{array}$ & $\begin{array}{l}3, \mathrm{CC} \\
4-4 \\
\end{array}$ & $\begin{array}{l}26.40 \\
31.23 \\
\end{array}$ & & & $\begin{array}{l}3, \mathrm{CC} \\
4, \mathrm{CC} \\
\end{array}$ & $\begin{array}{l}29.66 \\
39.08 \\
\end{array}$ \\
\hline & & $\mathrm{T}$ & $\begin{array}{r}\text { Carpocanopsis } \\
\text { cristata s.s. }\end{array}$ & $\begin{array}{l}4-4 \\
4, \mathrm{CC} \\
\end{array}$ & $\begin{array}{l}31.23 \\
34.93\end{array}$ & & & $\begin{array}{l}3, \mathrm{CC} \\
4, \mathrm{CC} \\
\end{array}$ & $\begin{array}{l}29.66 \\
39.08 \\
\end{array}$ \\
\hline & & B & $\begin{array}{r}\text { Botryostrobus } \\
\text { bramlettei }\end{array}$ & $\begin{array}{l}5-3 \\
5, \mathrm{CC}\end{array}$ & $\begin{array}{l}38.43 \\
44.55\end{array}$ & & & $\begin{array}{l}3, \mathrm{CC} \\
4, \mathrm{CC}\end{array}$ & $\begin{array}{l}29.66 \\
39.08 \\
\end{array}$ \\
\hline & 11.8 & $\mathrm{~T}$ & $\begin{array}{c}\text { Cyrtocapsella } \\
\text { cornuta }\end{array}$ & $\begin{array}{l}5-3 \\
5, \mathrm{CC}\end{array}$ & $\begin{array}{r}38.43 \\
44.55\end{array}$ & & & $\begin{array}{l}3, \mathrm{CC} \\
4, \mathrm{CC}\end{array}$ & $\begin{array}{l}29.66 \\
39.08\end{array}$ \\
\hline & [12.5] & $\mathrm{T}$ & $\begin{array}{c}\text { Cyrtocapsella } \\
\text { tetrapera }\end{array}$ & $\begin{array}{l}5-3 \\
5, \mathrm{CC} \\
\end{array}$ & $\begin{array}{l}38.43 \\
44.55 \\
\end{array}$ & & & $\begin{array}{l}3, \mathrm{CC} \\
4, \mathrm{CC} \\
\end{array}$ & $\begin{array}{l}29.66 \\
39.08 \\
\end{array}$ \\
\hline & & $\mathrm{T}$ & $\begin{array}{r}\text { Lithopera } \\
\text { renzae }\end{array}$ & $\begin{array}{l}5-3 \\
5, \mathrm{CC}\end{array}$ & $\begin{array}{l}38.43 \\
44.55 \\
\end{array}$ & & & {$\left[\begin{array}{l}6, \mathrm{CC} \\
7, \mathrm{CC}\end{array}\right]$} & $\left.\begin{array}{l}55.95 \\
64.74\end{array}\right]$ \\
\hline
\end{tabular}


Table 4. (Continued).

\begin{tabular}{|c|c|c|c|c|c|c|c|c|c|}
\hline \multirow[b]{2}{*}{ Zone } & \multirow[b]{2}{*}{$\begin{array}{l}\text { Age } \\
(\mathrm{Ma})\end{array}$} & \multirow[b]{2}{*}{$\begin{array}{c}\text { Top } \\
\text { or } \\
\text { bottom }\end{array}$} & \multirow[b]{2}{*}{ Species } & \multicolumn{2}{|c|}{ Hole 575} & \multicolumn{2}{|c|}{ Hole 575A } & \multicolumn{2}{|c|}{ Hole 575B } \\
\hline & & & & Core-Section & $\begin{array}{l}\text { Sub-bottom } \\
\text { depth } \\
\text { (m) }\end{array}$ & Core-Section & $\begin{array}{l}\text { Sub-bottom } \\
\text { depth } \\
\text { (m) }\end{array}$ & Core-Section & $\begin{array}{l}\text { Sub-bottom } \\
\text { depth } \\
\text { (m) }\end{array}$ \\
\hline \multirow[t]{5}{*}{ D. petterssoni } & \multirow[t]{4}{*}{11.8} & $\mathrm{~T}$ & $\begin{array}{l}\text { Dorcadospyris } \\
\quad \text { alata }\end{array}$ & $\begin{array}{l}5-3 \\
5, \mathrm{CC}\end{array}$ & $\begin{array}{l}38.43 \\
44.55\end{array}$ & & & {$\left[\begin{array}{l}8, \mathrm{CC} \\
9, \mathrm{CC}\end{array}\right.$} & $\left.\begin{array}{l}73.74 \\
81.33\end{array}\right]$ \\
\hline & & $\mathrm{T}$ & $\begin{array}{c}\text { Giraffospyris } \\
\text { toxaria }\end{array}$ & $\begin{array}{l}5-3 \\
5, \mathrm{CC}\end{array}$ & $\begin{array}{l}38.43 \\
44.55\end{array}$ & & & $\begin{array}{l}5, \mathrm{CC} \\
6, \mathrm{CC}\end{array}$ & $\begin{array}{l}48.28 \\
55.95\end{array}$ \\
\hline & & B & $\begin{array}{c}\text { Cyrtocapsella } \\
\text { japonica }\end{array}$ & {$\left[\begin{array}{l}5, \mathrm{CC} \\
6-3\end{array}\right.$} & $\left.\begin{array}{l}44.55 \\
47.92\end{array}\right]$ & 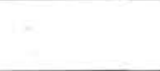 & - & $\begin{array}{l}5, \mathrm{CC} \\
6, \mathrm{CC}\end{array}$ & $\begin{array}{l}48.28 \\
55.95\end{array}$ \\
\hline & & $\mathrm{T}$ & $\begin{array}{l}\text { Didymocyrtis } \\
\text { mammifera }\end{array}$ & $\begin{array}{l}5-3 \\
5, \mathrm{CC}\end{array}$ & $\begin{array}{l}38.43 \\
44.55 \\
\end{array}$ & & & {$\left[\begin{array}{l}7, \mathrm{CC} \\
8, \mathrm{CC}\end{array}\right.$} & $\left.\begin{array}{l}64.73 \\
73.74\end{array}\right]$ \\
\hline & 12.3 & B & $\begin{array}{l}\text { Diartus } \\
\quad \text { petterssoni }\end{array}$ & $\begin{array}{l}5-3 \\
5, \mathrm{CC}\end{array}$ & $\begin{array}{l}38.43 \\
44.55\end{array}$ & & & $\begin{array}{l}5, \mathrm{CC} \\
6, \mathrm{CC}\end{array}$ & $\begin{array}{l}48.28 \\
55.95 \\
\end{array}$ \\
\hline \multirow[t]{17}{*}{ D. alata } & \multirow[t]{4}{*}{14.2} & B & $\begin{array}{l}\text { Lithopera } \\
\text { neotera }\end{array}$ & {$\left[\begin{array}{l}7, \mathrm{CC} \\
8-3\end{array}\right.$} & $\left.\begin{array}{l}63.54 \\
66.92\end{array}\right]$ & & & {$\left[\begin{array}{l}7, \mathrm{CC} \\
8, \mathrm{CC}\end{array}\right.$} & $\left.\begin{array}{l}64.73 \\
73.74\end{array}\right]$ \\
\hline & & B & $\begin{array}{l}\text { Lithopera } \\
\text { thornburgi }\end{array}$ & $\begin{array}{l}6-3 \\
6, \mathrm{CC}\end{array}$ & $\begin{array}{l}47.92 \\
53.98\end{array}$ & & & {$\left[\begin{array}{l}7, \mathrm{CC} \\
8, \mathrm{CC}\end{array}\right.$} & $\left.\begin{array}{l}64.73 \\
73.74\end{array}\right]$ \\
\hline & & B & $\begin{array}{l}\text { Phormostichoartus } \\
\text { corbula }\end{array}$ & $\begin{array}{l}6, \mathrm{CC} \\
7-3\end{array}$ & $\begin{array}{l}53.98 \\
57.42 \\
\end{array}$ & & & {$\left[\begin{array}{l}6, \mathrm{CC} \\
7, \mathrm{CC}\end{array}\right.$} & $\left.\begin{array}{l}55.95 \\
64.73\end{array}\right]$ \\
\hline & & B & $\begin{array}{l}\text { Dictyocoryne } \\
\text { ontongensis }\end{array}$ & $\begin{array}{l}6, \mathrm{CC} \\
7-3\end{array}$ & $\begin{array}{l}53.98 \\
57.42 \\
\end{array}$ & & & {$\left[\begin{array}{l}6, \mathrm{CC} \\
7, \mathrm{CC}\end{array}\right.$} & $\left.\begin{array}{l}55.95 \\
64.73\end{array}\right]$ \\
\hline & \multirow[t]{2}{*}[13.8]{} & B & $\begin{array}{c}\text { Didymocyrtis } \\
\text { laticonus }\end{array}$ & $\begin{array}{l}6, \mathrm{CC} \\
7-3 \\
\end{array}$ & $\begin{array}{l}53.98 \\
57.42 \\
\end{array}$ & & & $\begin{array}{l}8, \mathrm{CC} \\
9, \mathrm{CC} \\
\end{array}$ & $\begin{array}{l}73.74 \\
81.33 \\
\end{array}$ \\
\hline & & $\mathrm{T}$ & $\begin{array}{l}\text { Liriospyris } \\
\text { parkerae }\end{array}$ & $\begin{array}{l}8-3 \\
8, \mathrm{CC}\end{array}$ & $\begin{array}{l}66.92 \\
72.99 \\
\end{array}$ & & & $\begin{array}{l}8, \mathrm{CC} \\
9, \mathrm{CC} \\
\end{array}$ & $\begin{array}{l}73.74 \\
81.33 \\
\end{array}$ \\
\hline & \multirow[t]{3}{*}{14.75} & $\mathrm{~T}$ & $\begin{array}{c}\text { Calocycletta } \\
\text { virginis }\end{array}$ & $\begin{array}{l}8-3 \\
8, \mathrm{CC}\end{array}$ & $\begin{array}{l}66.92 \\
72.99 \\
\end{array}$ & & & $\begin{array}{l}8, \mathrm{CC} \\
9, \mathrm{CC} \\
\end{array}$ & $\begin{array}{l}73.74 \\
81.33 \\
\end{array}$ \\
\hline & & $\mathrm{T}$ & $\begin{array}{c}\text { Carpocanopsis } \\
\text { bramlettei }\end{array}$ & $\begin{array}{l}8-3 \\
8, \mathrm{CC} \\
\end{array}$ & $\begin{array}{l}66.92 \\
72.99 \\
\end{array}$ & & & {$\left[\begin{array}{l}10, \mathrm{CC} \\
11, \mathrm{CC}\end{array}\right.$} & $\left.\begin{array}{l}90.98 \\
99.53\end{array}\right]$ \\
\hline & & $\mathrm{T}$ & $\begin{array}{l}\text { Acrocubus } \\
\text { octopylus }\end{array}$ & $\begin{array}{l}8-3 \\
8, \mathrm{CC}\end{array}$ & $\begin{array}{l}66.92 \\
72.99 \\
\end{array}$ & & & $\begin{array}{l}9, \mathrm{CC} \\
10, \mathrm{CC}\end{array}$ & $\begin{array}{l}81.33 \\
90.98 \\
\end{array}$ \\
\hline & \multirow[t]{3}{*}{15.35} & $\mathrm{~T}$ & $\begin{array}{c}\text { Calocycletta } \\
\text { costata }\end{array}$ & $\begin{array}{l}8, C C \\
9-3\end{array}$ & $\begin{array}{l}72.99 \\
76.42 \\
\end{array}$ & & & $\begin{array}{l}9, \mathrm{CC} \\
10, \mathrm{CC}\end{array}$ & $\begin{array}{l}81.33 \\
90.98 \\
\end{array}$ \\
\hline & & $\mathrm{T}$ & $\begin{array}{c}\text { Didymocyrtis } \\
\text { tubaria }\end{array}$ & $\begin{array}{l}8, \mathrm{CC} \\
9-3\end{array}$ & $\begin{array}{l}72.99 \\
76.42 \\
\end{array}$ & & & $\begin{array}{l}10, \mathrm{CC} \\
11, \mathrm{CC}\end{array}$ & $\begin{array}{l}90.98 \\
99.53 \\
\end{array}$ \\
\hline & & $\mathrm{T}$ & $\begin{array}{c}\text { Didymocyrtis } \\
\text { violina }\end{array}$ & $\begin{array}{l}8, C C \\
9-3\end{array}$ & $\begin{array}{l}72.99 \\
76.42 \\
\end{array}$ & & & $\begin{array}{l}10, \mathrm{CC} \\
11, \mathrm{CC} \\
\end{array}$ & $\begin{array}{l}90.98 \\
99.53 \\
\end{array}$ \\
\hline & 15.8 & $\mathrm{~T}$ & $\begin{array}{c}\text { Dorcadospyris } \\
\text { dentata }\end{array}$ & $\begin{array}{l}8, \mathrm{CC} \\
9-3\end{array}$ & $\begin{array}{l}72.99 \\
76.42 \\
\end{array}$ & & & {$\left[\begin{array}{l}11, \mathrm{CC} \\
12, \mathrm{CC}\end{array}\right.$} & $\left.\begin{array}{l}99.53 \\
90.98\end{array}\right]$ \\
\hline & & $\mathrm{T}$ & $\begin{array}{l}\text { Liriospyris } \\
\text { stauropora }\end{array}$ & $\begin{array}{l}8, C C \\
9-3\end{array}$ & $\begin{array}{l}72.99 \\
76.42 \\
\end{array}$ & & & $\begin{array}{l}10, \mathrm{CC} \\
11, \mathrm{CC} \\
\end{array}$ & $\begin{array}{l}90.98 \\
99.53 \\
\end{array}$ \\
\hline & & & $\begin{array}{l}\text { L. stauropora } \\
\rightarrow \text { L. parkeri }\end{array}$ & $\begin{array}{l}8, \mathrm{CC} \\
10, \mathrm{CC}\end{array}$ & $\begin{array}{l}72.99 \\
89.01\end{array}$ & & & $\begin{array}{l}10, \mathrm{CC} \\
13, \mathrm{CC}\end{array}$ & $\begin{array}{r}90.98 \\
114.04 \\
\end{array}$ \\
\hline & & $\mathrm{T}$ & $\begin{array}{c}\text { Dorcadospyris } \\
\text { forcipata }\end{array}$ & $\begin{array}{l}9-3 \\
9, \mathrm{CC}\end{array}$ & $\begin{array}{l}76.42 \\
80.14 \\
\end{array}$ & & & $\begin{array}{l}11, \mathrm{CC} \\
12, \mathrm{CC}\end{array}$ & $\begin{array}{r}99.53 \\
109.15 \\
\end{array}$ \\
\hline & & & $\begin{array}{l}\text { D. dentata } \\
\rightarrow \text { D. alata }\end{array}$ & $\begin{array}{l}9-3 \\
9, \mathrm{CC} \\
\end{array}$ & $\begin{array}{l}76.42 \\
80.14 \\
\end{array}$ & & & $\begin{array}{l}11, \mathrm{CC} \\
12, \mathrm{CC}\end{array}$ & $\begin{array}{r}99.53 \\
109.15 \\
\end{array}$ \\
\hline C. costata & 15.7 & B & $\begin{array}{l}\text { Dorcadospyris } \\
\quad \text { alata }\end{array}$ & $\begin{array}{l}9, \mathrm{CC} \\
10-4 \\
\end{array}$ & $\begin{array}{l}80.14 \\
85.12 \\
\end{array}$ & & & $\begin{array}{l}11, \mathrm{CC} \\
12, \mathrm{CC}\end{array}$ & $\begin{array}{r}99.53 \\
109.15 \\
\end{array}$ \\
\hline & & $\mathbf{T}$ & $\begin{array}{c}\text { Eucyrtidium } \\
\text { diaphanes }\end{array}$ & $\begin{array}{l}10-4 \\
10, \mathrm{CC}\end{array}$ & $\begin{array}{l}85.12 \\
89.01 \\
\end{array}$ & & & $\begin{array}{l}11, \mathrm{CC} \\
12, \mathrm{CC}\end{array}$ & $\begin{array}{r}99.53 \\
109.15 \\
\end{array}$ \\
\hline & & B & $\begin{array}{l}\text { Liriospyris } \\
\text { parkerae }\end{array}$ & $\begin{array}{l}10-4 \\
10, \mathrm{CC}\end{array}$ & $\begin{array}{l}85.12 \\
89.01 \\
\end{array}$ & & & $\begin{array}{l}12, \mathrm{CC} \\
13, \mathrm{CC}\end{array}$ & $\begin{array}{l}109.15 \\
114.04 \\
\end{array}$ \\
\hline & & $T$ & $\begin{array}{c}\text { Carpocanopsis } \\
\text { cingulata }\end{array}$ & $\begin{array}{l}10-4 \\
10, \mathrm{CC}\end{array}$ & $\begin{array}{l}85.12 \\
89.01\end{array}$ & & & $\begin{array}{l}12, \mathrm{CC} \\
13, \mathrm{CC}\end{array}$ & $\begin{array}{l}109.15 \\
114.04 \\
\end{array}$ \\
\hline & 16.2 & $\mathrm{~T}$ & $\begin{array}{r}\text { Didymocyrtis } \\
\text { prismatica }\end{array}$ & $\begin{array}{l}11-3 \\
11, \mathrm{CC}\end{array}$ & $\begin{array}{l}92.51 \\
98.45 \\
\end{array}$ & & & $\begin{array}{l}12, \mathrm{CC} \\
13, \mathrm{CC}\end{array}$ & $\begin{array}{l}109.15 \\
114.04 \\
\end{array}$ \\
\hline & & B & $\begin{array}{r}\text { Carpocanopsis } \\
\text { cristata s.s. }\end{array}$ & [- & & & & {$[-$} & \\
\hline
\end{tabular}


Table 4. (Continued).

\begin{tabular}{|c|c|c|c|c|c|c|c|c|c|}
\hline \multirow[b]{2}{*}{ Zone } & \multirow[b]{2}{*}{$\begin{array}{l}\text { Age } \\
\text { (Ma) }\end{array}$} & \multirow[b]{2}{*}{$\begin{array}{l}\text { Top } \\
\text { or } \\
\text { bottom }\end{array}$} & \multirow[b]{2}{*}{ Species } & \multicolumn{2}{|c|}{ Hole 575} & \multicolumn{2}{|c|}{ Hole 575A } & \multicolumn{2}{|c|}{ Hole 575B } \\
\hline & & & & Core-Section & $\begin{array}{l}\text { Sub-bottom } \\
\text { depth } \\
\text { (m) }\end{array}$ & Core-Section & $\begin{array}{l}\text { Sub-bottom } \\
\text { depth } \\
\text { (m) }\end{array}$ & Core-Section & $\begin{array}{l}\text { Sub-bottom } \\
\text { depth } \\
\text { (m) }\end{array}$ \\
\hline \multirow[t]{7}{*}{ C. costata } & & $\mathrm{T}$ & $\begin{array}{l}\text { Carpocanopsis } \\
\text { favosa }\end{array}$ & $\begin{array}{l}11-3 \\
11, \mathrm{CC}\end{array}$ & $\begin{array}{l}92.51 \\
98.45\end{array}$ & $\begin{array}{l}1-2 \\
1, \mathrm{CC}\end{array}$ & $\begin{array}{l}95.72 \\
98.86\end{array}$ & $\begin{array}{l}12, \mathrm{CC} \\
13, \mathrm{CC}\end{array}$ & $\begin{array}{l}114.04 \\
114.04\end{array}$ \\
\hline & & B & $\begin{array}{r}\text { Lithopera } \\
\text { renzae }\end{array}$ & & & $\begin{array}{l}1-1 \\
1-2\end{array}$ & $\begin{array}{l}94.22 \\
95.72\end{array}$ & \multicolumn{2}{|c|}{$[-]$} \\
\hline & & B & $\begin{array}{l}\text { Acrocubus } \\
\text { octopylus }\end{array}$ & & & $\begin{array}{l}1-2 \\
1, \mathrm{CC}\end{array}$ & $\begin{array}{l}95.72 \\
98.86\end{array}$ & $\begin{array}{l}13, \mathrm{CC} \\
14, \mathrm{CC}\end{array}$ & $\begin{array}{l}114.04 \\
118.97\end{array}$ \\
\hline & & B & $\begin{array}{c}\text { Giraffospyris } \\
\text { toxaria }\end{array}$ & . & & $\begin{array}{l}1-2 \\
1, \mathrm{CC}\end{array}$ & $\begin{array}{l}95.72 \\
98.86\end{array}$ & $\begin{array}{l}13, \mathrm{CC} \\
14, \mathrm{CC}\end{array}$ & $\begin{array}{l}114.04 \\
114.04\end{array}$ \\
\hline & \multirow[t]{2}{*}{16.5} & $T$ & $\begin{array}{c}\text { Lychnocanoma } \\
\text { elongata }\end{array}$ & & & $\begin{array}{l}2, \mathrm{CC} \\
3, \mathrm{CC}\end{array}$ & $\begin{array}{l}102.26 \\
105.28\end{array}$ & & \\
\hline & & B & $\begin{array}{l}\text { Didymocyrtis } \\
\text { mammifera }\end{array}$ & & & $\begin{array}{l}2, \mathrm{CC} \\
3, \mathrm{CC}\end{array}$ & $\begin{array}{l}102.26 \\
105.28\end{array}$ & & \\
\hline & 17.3 & B & $\begin{array}{c}\text { Calocycletta } \\
\text { costata }\end{array}$ & & & $\begin{array}{l}4, \mathrm{CC} \\
5, \mathrm{CC}\end{array}$ & $\begin{array}{l}109.87 \\
114.00\end{array}$ & & \\
\hline \multirow[t]{5}{*}{ S. wolffii } & \multirow[t]{3}{*}{17.1} & B & $\begin{array}{c}\text { Dorcadospyris } \\
\text { dentata }\end{array}$ & & & $\begin{array}{l}5, \mathrm{CC} \\
6, \mathrm{CC}\end{array}$ & $\begin{array}{l}114.00 \\
119.17\end{array}$ & & \\
\hline & & B & $\begin{array}{c}\text { Calocycletta } \\
\text { caepa }\end{array}$ & & & $\begin{array}{l}5, \mathrm{CC} \\
6, \mathrm{CC}\end{array}$ & $\begin{array}{l}114.00 \\
119.17\end{array}$ & & \\
\hline & & B & $\begin{array}{l}\text { Liriospyris } \\
\quad \text { stauropora }\end{array}$ & & & $\begin{array}{l}7, \mathrm{CC} \\
8, \mathrm{CC}\end{array}$ & $\begin{array}{l}123.25 \\
126.29\end{array}$ & & \\
\hline & {$[20.4]$} & $\mathrm{T}$ & $\begin{array}{c}\text { Dorcadospyris } \\
\text { ateuchus }\end{array}$ & & & $\begin{array}{l}9, \mathrm{CC} \\
10, \mathrm{CC}\end{array}$ & $\begin{array}{l}128.14 \\
132.01\end{array}$ & & \\
\hline & 17.6 & B & $\begin{array}{c}\text { Stichocorys } \\
\text { wolffii }\end{array}$ & & & $\begin{array}{l}9, \mathrm{CC} \\
10, \mathrm{CC}\end{array}$ & $\begin{array}{l}128.14 \\
132.01\end{array}$ & & \\
\hline \multirow[t]{5}{*}{ S. delmontensis } & & B & $\begin{array}{l}\text { Siphostichartus } \\
\text { corona }\end{array}$ & & & $\begin{array}{l}9, \mathrm{CC} \\
10, \mathrm{CC}\end{array}$ & $\begin{array}{l}128.14 \\
132.01\end{array}$ & & \\
\hline & \multirow[t]{2}{*}{19.25} & B & $\begin{array}{c}\text { Didymocyrtis } \\
\text { violina }\end{array}$ & & & $\begin{array}{l}25, \mathrm{CC} \\
26, \mathrm{CC}\end{array}$ & $\begin{array}{l}181.96 \\
184.80\end{array}$ & & \\
\hline & & B & $\begin{array}{c}\text { Didymocyrtis } \\
\text { tubaria }\end{array}$ & & & $\begin{array}{l}25, \mathrm{CC} \\
26, \mathrm{CC}\end{array}$ & $\begin{array}{l}181.96 \\
184.88\end{array}$ & & \\
\hline & \multirow[t]{2}{*}{20.3} & B & $\begin{array}{l}\text { Stichocorys } \\
\quad \text { delmontensis }\end{array}$ & & & $\begin{array}{l}25, \mathrm{CC} \\
26, \mathrm{CC}\end{array}$ & $\begin{array}{l}181.96 \\
184.80\end{array}$ & & \\
\hline & & B & $\begin{array}{c}\text { Carpocanopsis } \\
\text { bramlettei }\end{array}$ & & & $\begin{array}{l}26, \mathrm{CC} \\
27, \mathrm{CC}\end{array}$ & $\begin{array}{l}184.80 \\
188.41\end{array}$ & & \\
\hline \multirow[t]{3}{*}{ C. tetrapera } & \multirow[t]{2}{*}{21.3} & $\mathrm{~T}$ & $\begin{array}{r}\text { Theocyrtis } \\
\text { annosa }\end{array}$ & & & $\begin{array}{l}31, \mathrm{CC} \\
32, \mathrm{CC}\end{array}$ & $\begin{array}{l}201.75 \\
205.13\end{array}$ & & \\
\hline & & $\mathrm{T}$ & $\begin{array}{c}\text { Calocycletta } \\
\text { robusta }\end{array}$ & & & $\begin{array}{l}31, \mathrm{CC} \\
32, \mathrm{CC}\end{array}$ & $\begin{array}{l}201.75 \\
205.13\end{array}$ & & \\
\hline & 21.35 & B & $\begin{array}{c}\text { Calocycletta } \\
\text { virginis }\end{array}$ & & & $\begin{array}{l}32, \mathrm{CC} \\
33, \mathrm{CC}\end{array}$ & $\begin{array}{l}205.13 \\
208.30\end{array}$ & & \\
\hline
\end{tabular}

Note: Pairs of levels and brackets as defined in Table 1 of this appendix. 


\section{APPENDIX B}

\section{Raw Data for the Leg 85 Holes}

The following appendix comprises tables of raw data for each of the Leg 85 holes. Unlike other DSDP reports (e.g., Westberg and Riedel, 1982) this study does not record species abundances, but rather notes only presence or absence. Radiolarian abundances and conditions of preservation are almost always "common" and "good." Exceptions to this general rule are noted in the text for each site.

Table 1. Raw data for Hole 572A.

\begin{tabular}{|c|c|c|c|c|c|c|c|c|c|c|c|c|c|c|c|c|c|c|c|c|c|c|c|c|c|c|c|c|c|c|}
\hline $\begin{array}{l}\text { Core-Section } \\
\text { (interval in cm) }\end{array}$ & 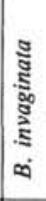 & 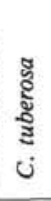 & $\frac{:}{\vdots}$ & 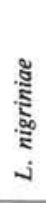 & 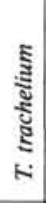 & 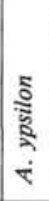 & 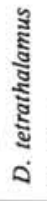 & 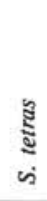 & 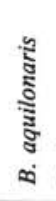 & $\begin{array}{l}\mathcal{Z} \\
\tilde{\Xi} \\
\dot{0} \\
\dot{v}\end{array}$ & 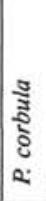 & $\begin{array}{l}\text { हैँ } \\
\text { हे } \\
\text { ம }\end{array}$ & 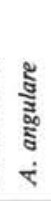 & 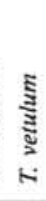 & $\begin{array}{l}\text { है } \\
\text { वे }\end{array}$ & 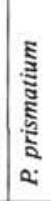 & 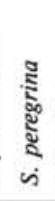 & $\underbrace{\stackrel{5}{5}}_{Q}$ & $\begin{array}{l}\text { y } \\
\text { ¿ } \\
\dot{y}\end{array}$ & 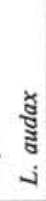 & 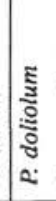 & 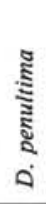 & 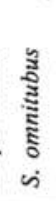 & 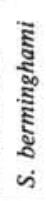 & 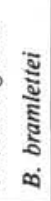 & 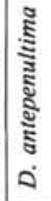 & 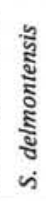 & 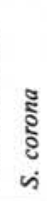 & 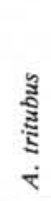 & $\begin{array}{l}\text { ปั } \\
\text { ปั }\end{array}$ \\
\hline $\begin{array}{l}1-1,108-109 \\
1-3,69-70 \\
1, C C \\
2-3,70-71 \\
2-5,70-71\end{array}$ & - & $\begin{array}{l}P \\
P \\
+ \\
-\end{array}$ & $\begin{array}{l}\mathrm{P} \\
\mathrm{P} \\
\mathrm{P} \\
\mathrm{P}\end{array}$ & $\begin{array}{l}\mathrm{P} \\
\mathrm{P} \\
\mathrm{P} \\
\mathrm{P}\end{array}$ & $\begin{array}{l}\mathrm{P} \\
\mathrm{P} \\
\mathrm{P}\end{array}$ & $\begin{array}{l}\mathrm{P} \\
\mathrm{P} \\
\mathrm{P} \\
\mathrm{P}\end{array}$ & $\begin{array}{l}\mathrm{P} \\
\mathrm{P} \\
\mathrm{P} \\
\mathrm{P} \\
\mathrm{P}\end{array}$ & $\begin{array}{l}\mathrm{P} \\
\mathrm{P} \\
\mathrm{P} \\
\mathrm{P}\end{array}$ & $\begin{array}{l}\mathrm{P} \\
\mathrm{P} \\
\mathrm{P} \\
\mathrm{P}\end{array}$ & P & $\begin{array}{l}\mathrm{P} \\
\mathrm{P} \\
\mathrm{P} \\
\mathrm{P}\end{array}$ & $\begin{array}{l}\bar{P} \\
P\end{array}$ & $\overline{-}$ & $\overline{-}$ & $\overline{-}$ & & & & & & & & & & & & & & & \\
\hline $\begin{array}{l}2, \mathrm{CC} \\
3-3,90-92 \\
3-5,90-92 \\
3, \mathrm{CC} \\
4-3,70-71\end{array}$ & & & - & $\begin{array}{l}+ \\
- \\
-\end{array}$ & $\begin{array}{l}\mathrm{P} \\
- \\
+ \\
-\end{array}$ & $\begin{array}{l}\mathrm{P} \\
\mathrm{P} \\
\mathrm{P}\end{array}$ & $\begin{array}{l}\mathrm{P} \\
\mathrm{P}\end{array}$ & $\begin{array}{l}\mathrm{P} \\
\mathrm{P}\end{array}$ & $\begin{array}{l}\mathrm{P} \\
\mathrm{P} \\
\mathrm{P}\end{array}$ & $\begin{array}{l}\mathrm{P} \\
\mathrm{P} \\
- \\
\mathrm{P}\end{array}$ & $\begin{array}{l}\mathrm{P} \\
\mathrm{P} \\
\mathrm{P} \\
\mathrm{P}\end{array}$ & $\begin{array}{l}\mathrm{P} \\
\mathrm{P} \\
\mathrm{P}\end{array}$ & $\begin{array}{l}\mathrm{P} \\
\mathrm{P} \\
\mathrm{P} \\
-\end{array}$ & $\begin{array}{l}\mathrm{P} \\
\mathrm{P} \\
\mathrm{P} \\
\mathrm{P} \\
\mathrm{P}\end{array}$ & $\begin{array}{l}+ \\
+ \\
+\end{array}$ & $\begin{array}{l}\mathrm{P} \\
\mathrm{P}\end{array}$ & & & - & & & & & & & & & & & \\
\hline $\begin{array}{l}4, C C \\
5-3,70-71 \\
5, C C \\
6-3,70-71 \\
6, C C\end{array}$ & & & - & + & - & $\begin{array}{l}\mathrm{P} \\
\mathrm{P} \\
\mathrm{P} \\
\mathrm{P} \\
\mathrm{P}\end{array}$ & $\begin{array}{l}\mathrm{P} \\
\mathrm{P} \\
\mathrm{P} \\
+\end{array}$ & $\begin{array}{l}\mathrm{P} \\
\mathrm{P} \\
\mathrm{P} \\
\mathrm{P}\end{array}$ & $\begin{array}{l}\mathrm{P} \\
\mathrm{P} \\
\mathrm{P} \\
\mathrm{P} \\
\mathrm{P}\end{array}$ & $\begin{array}{l}\mathrm{P} \\
\mathrm{P} \\
\mathrm{P}\end{array}$ & $\begin{array}{l}\mathrm{P} \\
\mathrm{P} \\
\mathrm{P} \\
\mathrm{P} \\
\mathrm{P}\end{array}$ & $\begin{array}{l}\mathrm{P} \\
\mathrm{P} \\
\mathrm{P} \\
\mathrm{P} \\
\mathrm{P}\end{array}$ & & $\begin{array}{l}\mathrm{P} \\
\mathrm{P} \\
\mathrm{P} \\
\mathrm{P} \\
\mathrm{P}\end{array}$ & $\begin{array}{l}+ \\
+ \\
\text { P }\end{array}$ & $\begin{array}{l}\mathrm{P} \\
\mathrm{P} \\
\mathrm{P} \\
\mathrm{P} \\
\mathrm{P}\end{array}$ & $\begin{array}{l}\bar{P} \\
P \\
P\end{array}$ & $\begin{array}{l}\bar{P} \\
P\end{array}$ & $\begin{array}{l}+ \\
\frac{-}{-} \\
+\end{array}$ & $\frac{+}{\mathrm{P}}$ & - & & - & & $\frac{-}{+}$ & & & & & \\
\hline $\begin{array}{l}7-3,70-71 \\
7, C C \\
8-3,70-71 \\
8, C C \\
9-3,70-71\end{array}$ & & & & & & - & - & $\begin{array}{l}P \\
+ \\
-\end{array}$ & $\begin{array}{l}- \\
-\end{array}$ & $\begin{array}{l}\mathrm{P} \\
\mathrm{P} \\
\mathrm{P}\end{array}$ & $\begin{array}{l}P \\
P\end{array}$ & $\begin{array}{l}\mathrm{P} \\
\mathrm{P}\end{array}$ & & $\begin{array}{l}\mathrm{P} \\
\mathrm{P} \\
\mathrm{P} \\
\mathrm{P} \\
\mathrm{P}\end{array}$ & $\begin{array}{l}\mathrm{P} \\
\mathrm{P} \\
-\end{array}$ & $\begin{array}{l}\mathrm{P} \\
\mathrm{P} \\
\mathrm{P} \\
\mathrm{P} \\
\mathrm{P}\end{array}$ & $\begin{array}{l}\mathrm{P} \\
\mathrm{P} \\
\mathrm{P} \\
\mathrm{P} \\
\mathrm{P}\end{array}$ & $\begin{array}{l}P \\
P \\
P \\
P\end{array}$ & $\begin{array}{l}+ \\
\mathrm{P} \\
- \\
+\end{array}$ & $\begin{array}{l}\mathrm{P} \\
+ \\
\mathrm{P} \\
+\end{array}$ & $\begin{array}{l}\mathrm{P} \\
\mathrm{P} \\
\mathrm{P} \\
\mathrm{P} \\
\mathrm{P}\end{array}$ & $\begin{array}{l}\bar{P} \\
P\end{array}$ & $\begin{array}{l}- \\
+ \\
P\end{array}$ & $\frac{-}{-}$ & $\begin{array}{l}- \\
-\end{array}$ & & & & & \\
\hline $\begin{array}{l}9, \mathrm{CC} \\
10, \mathrm{CC} \\
11-3,78-79 \\
11, \mathrm{CC} \\
12-3,68-69\end{array}$ & & & & & & & & & $\begin{array}{l}\mathrm{P} \\
\mathrm{P} \\
\mathrm{P} \\
\mathrm{P} \\
+\end{array}$ & $\begin{array}{l}\mathrm{P} \\
\mathrm{P} \\
\mathrm{P}\end{array}$ & $\begin{array}{l}\mathrm{P} \\
\mathrm{P} \\
\mathrm{P} \\
\mathrm{P}\end{array}$ & $\begin{array}{l}\mathrm{P} \\
\mathrm{P}\end{array}$ & & $\begin{array}{l}\mathrm{P} \\
\mathrm{P} \\
\mathrm{P}\end{array}$ & + & $\begin{array}{l}P \\
+ \\
- \\
+ \\
-\end{array}$ & $\begin{array}{l}\mathrm{P} \\
\mathrm{P} \\
\mathrm{P} \\
\mathrm{P}\end{array}$ & $\overline{+}$ & - & $\begin{array}{l}\mathrm{P} \\
\mathrm{P} \\
\mathrm{P} \\
\mathrm{P}\end{array}$ & $\begin{array}{l}P \\
P \\
P \\
P\end{array}$ & $\begin{array}{l}\mathrm{P} \\
\mathrm{P} \\
\mathrm{P} \\
\mathrm{P}\end{array}$ & $\begin{array}{l}\bar{P} \\
\bar{P}\end{array}$ & $\begin{array}{l}\mathrm{P} \\
\mathrm{P} \\
\mathrm{P} \\
\mathrm{P} \\
\mathrm{P}\end{array}$ & $\begin{array}{l}- \\
+ \\
+ \\
+\end{array}$ & & & & & \\
\hline $\begin{array}{l}12, \mathrm{CC} \\
13-3,70-71 \\
13, \mathrm{CC} \\
14-3,70-71 \\
14, \mathrm{CC}\end{array}$ & & & & & & & & & $\begin{array}{l}\mathrm{P} \\
\mathrm{P} \\
- \\
-\end{array}$ & $\begin{array}{l}\mathrm{P} \\
\mathrm{P} \\
\mathrm{P} \\
\mathrm{P}\end{array}$ & $\begin{array}{l}\mathrm{P} \\
\mathrm{P} \\
\mathrm{P} \\
\mathrm{P}\end{array}$ & $\begin{array}{l}\mathrm{P} \\
\mathrm{P} \\
\mathrm{P} \\
\mathrm{P} \\
\mathrm{P}\end{array}$ & & $\begin{array}{l}\mathrm{P} \\
\mathrm{P} \\
\mathrm{P}\end{array}$ & & $\begin{array}{l}- \\
- \\
- \\
-\end{array}$ & $\begin{array}{l}\mathrm{P} \\
\mathrm{P} \\
\mathrm{P} \\
\mathrm{P} \\
\mathrm{P}\end{array}$ & $\begin{array}{l}\mathrm{P} \\
\mathrm{P} \\
\mathrm{P}\end{array}$ & $\begin{array}{l}+ \\
+\end{array}$ & $\begin{array}{l}\mathrm{P} \\
\mathrm{P} \\
\mathrm{P} \\
\mathrm{P} \\
\mathrm{P}\end{array}$ & $\begin{array}{l}\mathrm{P} \\
\mathrm{P} \\
\mathrm{P} \\
\mathrm{P} \\
\mathrm{P}\end{array}$ & $\begin{array}{l}\mathrm{P} \\
\mathrm{P} \\
\mathrm{P} \\
\mathrm{P} \\
\mathrm{P}\end{array}$ & $\begin{array}{l}\mathrm{P} \\
\mathrm{P} \\
\mathrm{P} \\
\mathrm{P}\end{array}$ & $\begin{array}{l}- \\
\mathrm{P} \\
\mathrm{P} \\
\mathrm{P}\end{array}$ & $\begin{array}{l}+ \\
\mathrm{P} \\
+ \\
\mathrm{P} \\
-\end{array}$ & $\begin{array}{l}\frac{+}{P} \\
P\end{array}$ & $\begin{array}{l}\overline{-} \\
\mathrm{P} \\
\mathrm{P}\end{array}$ & $\begin{array}{l}- \\
\bar{P} \\
\mathrm{P} \\
\mathrm{P}\end{array}$ & $\frac{-}{-}$ & \\
\hline $\begin{array}{l}15-3,70-71 \\
15, \mathrm{CC} \\
16-3,72-73 \\
16, \mathrm{CC} \\
17, \mathrm{CC}\end{array}$ & & & & & & & & & & $\begin{array}{l}\mathrm{P} \\
\mathrm{P} \\
\mathrm{P} \\
\mathrm{P}\end{array}$ & $\begin{array}{l}\mathrm{P} \\
\mathrm{P} \\
\mathrm{P} \\
\mathrm{P} \\
\mathrm{P}\end{array}$ & $\begin{array}{l}\mathrm{P} \\
\mathrm{P} \\
\mathrm{P} \\
\mathrm{P}\end{array}$ & & $\begin{array}{l}\mathrm{P} \\
\mathrm{P} \\
\mathrm{P} \\
\mathrm{P}\end{array}$ & & - & $\begin{array}{l}\mathrm{P} \\
\mathrm{P} \\
\mathrm{P} \\
\mathrm{P} \\
\mathrm{P}\end{array}$ & $\begin{array}{l}\mathrm{P} \\
\mathrm{P} \\
\mathrm{P} \\
\mathrm{P}\end{array}$ & & $\begin{array}{l}\mathrm{P} \\
\mathrm{P} \\
\mathrm{P} \\
\mathrm{P}\end{array}$ & $\begin{array}{l}\mathrm{P} \\
\mathrm{P} \\
\mathrm{P} \\
\mathrm{P} \\
\mathrm{P}\end{array}$ & $\begin{array}{l}\mathrm{P} \\
\mathrm{P} \\
\mathrm{P} \\
\mathrm{P} \\
\mathrm{P}\end{array}$ & $\begin{array}{l}\mathrm{P} \\
\mathrm{P} \\
\mathrm{P} \\
\mathrm{P} \\
\mathrm{P}\end{array}$ & $\begin{array}{l}\mathrm{P} \\
\mathrm{P} \\
\mathrm{P} \\
\mathrm{P} \\
\mathrm{P}\end{array}$ & $\begin{array}{l}\mathrm{P} \\
\mathrm{P} \\
\mathrm{P} \\
\mathrm{P}\end{array}$ & $\begin{array}{l}\mathrm{P} \\
\mathrm{P} \\
\mathrm{P} \\
\mathrm{P} \\
\mathrm{P}\end{array}$ & $\begin{array}{l}\mathrm{P} \\
\mathrm{P} \\
\mathrm{P}\end{array}$ & $\begin{array}{l}\mathrm{P} \\
\mathrm{P} \\
\mathrm{P} \\
\mathrm{P} \\
\mathrm{P}\end{array}$ & $\begin{array}{l}\mathrm{P} \\
\mathrm{P} \\
- \\
\mathrm{P}\end{array}$ & $\begin{array}{l}- \\
\bar{P} \\
P\end{array}$ \\
\hline
\end{tabular}

Note: $\mathrm{P}=$ present; $+=$ rare (one or two specimens); $-=$ searched for and found to be absent. 


\section{A. NIGRINI}

Table 2. Raw data for Hole $572 \mathrm{C}$ (for explanation of symbols see note to this appendix, Table 1).

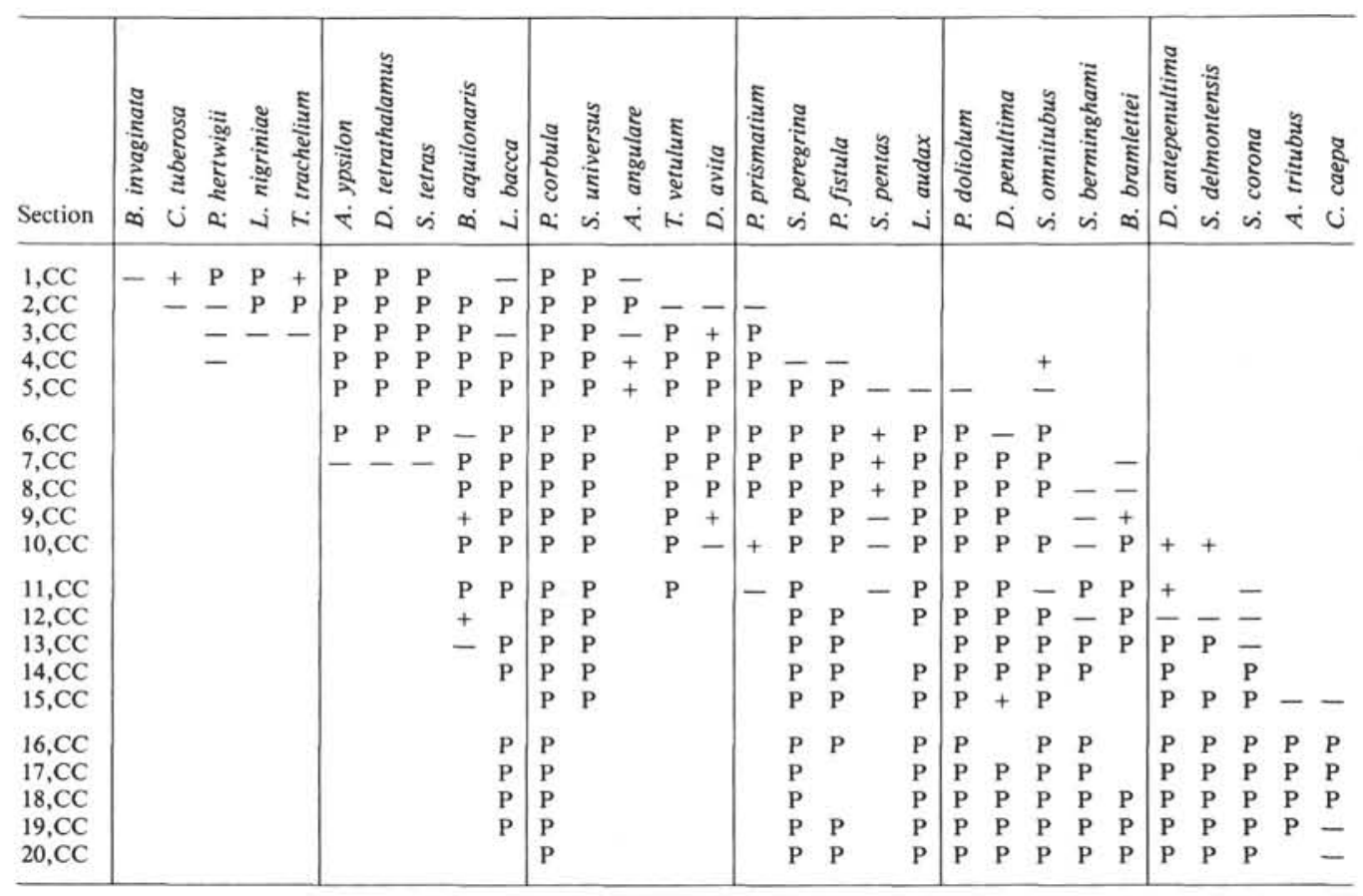


Table 3. Raw data for Hole 572D (for explanation of symbols see note to this appendix, Table 1).

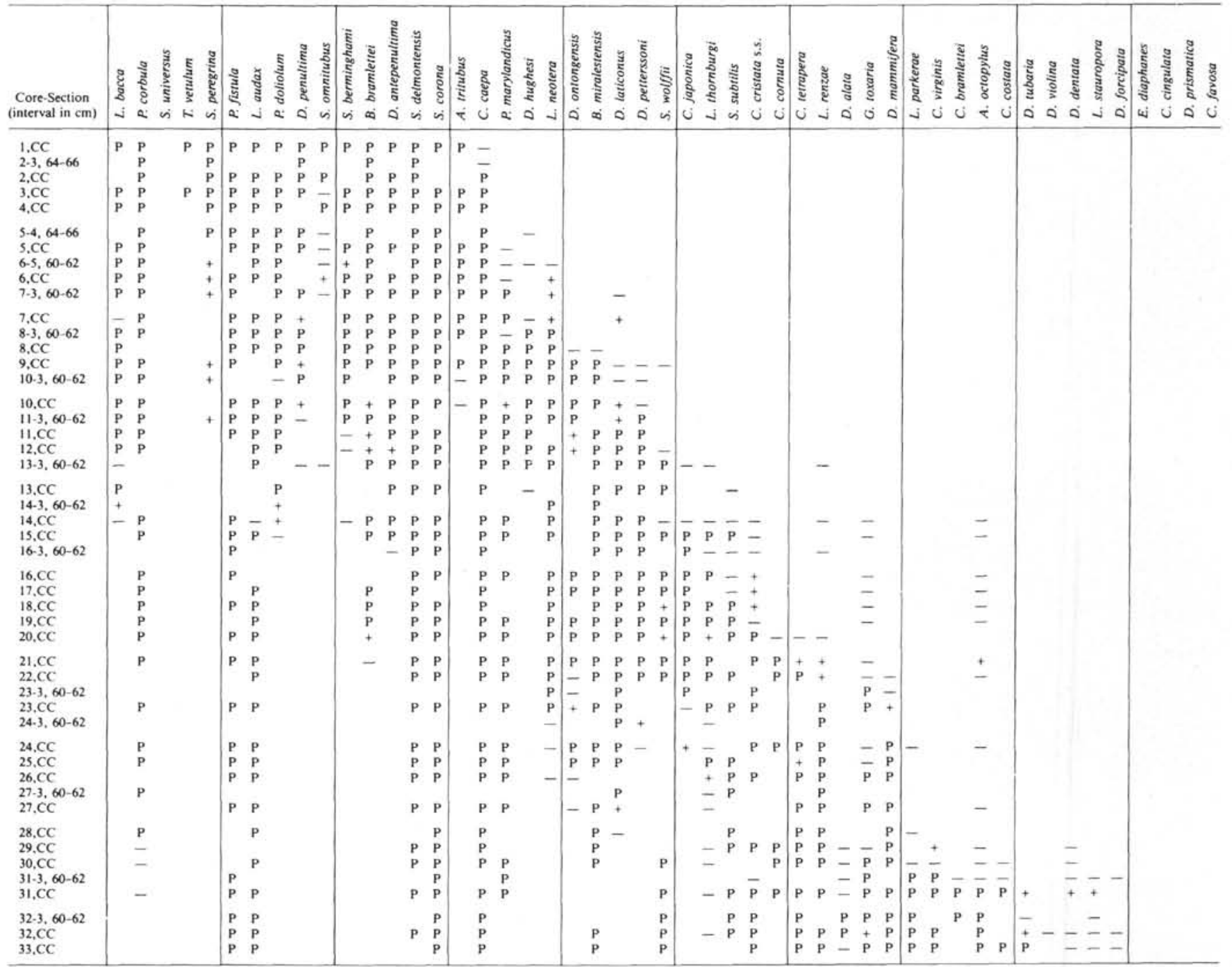


Table 4. Raw data for Hole 573 (for explanation of symbols see note to this appendix, Table 1).

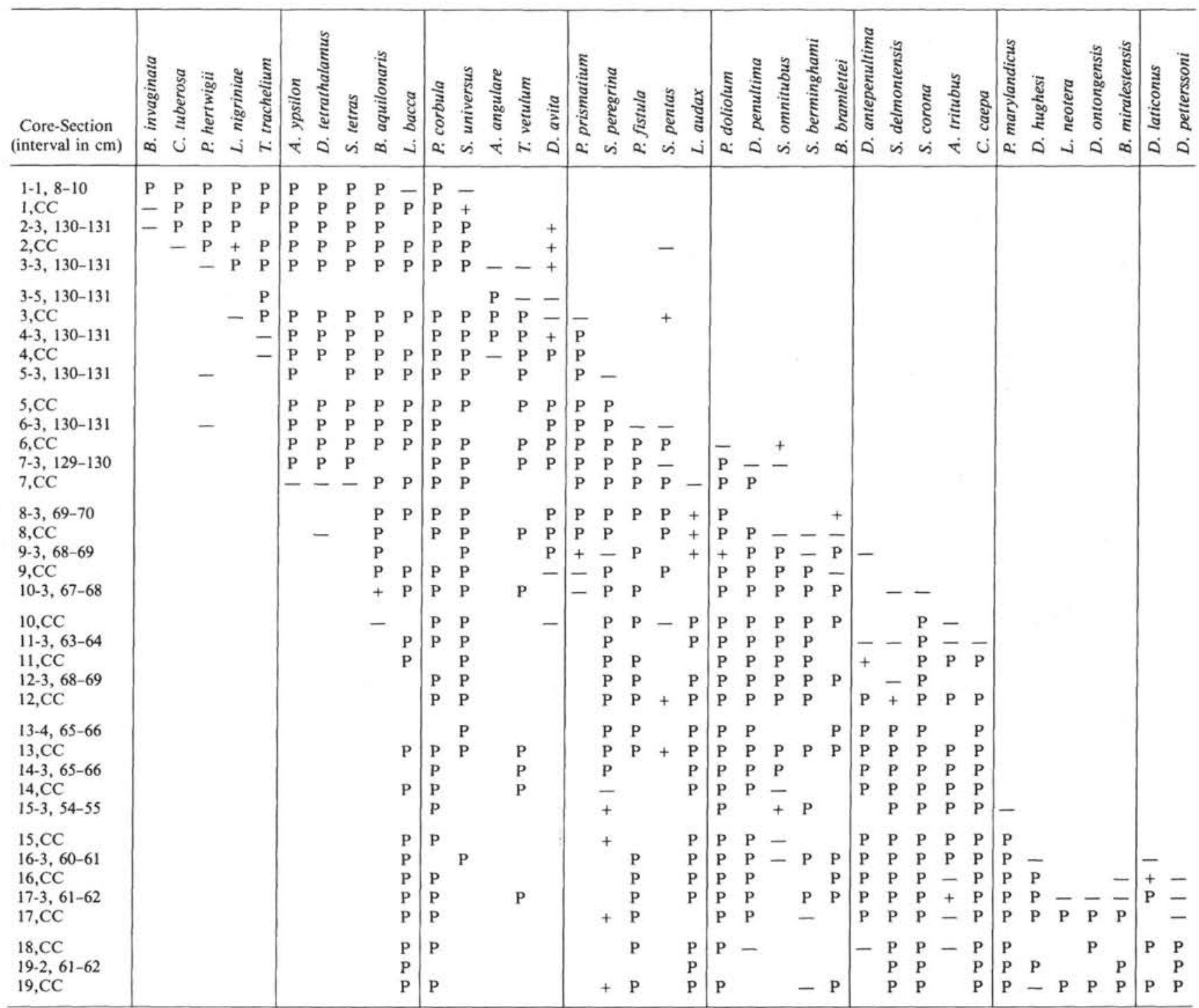

Table 5. Raw data for.Hole 572A (for explanation of symbols see note to this appendix, Table 1).

\begin{tabular}{|c|c|c|c|c|c|c|c|c|c|c|c|c|c|c|c|c|c|c|c|c|c|c|}
\hline Section & 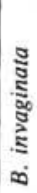 & 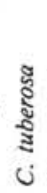 & 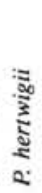 & 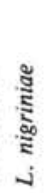 & 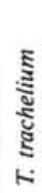 & 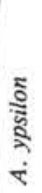 & 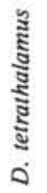 & $\begin{array}{l}\tilde{y} \\
\dot{z} \\
\dot{v}\end{array}$ & 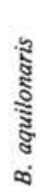 & $\begin{array}{l}\mathbb{Z} \\
\text { g } \\
\text { i }\end{array}$ & 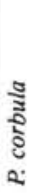 & 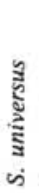 & 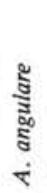 & 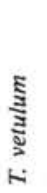 & $\begin{array}{l}\text { : } \\
\text { हे } \\
0\end{array}$ & 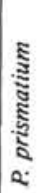 & 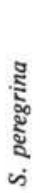 & $\underbrace{\stackrel{0}{3}}_{0}$ & 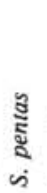 & 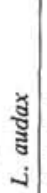 & 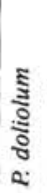 & 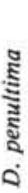 \\
\hline $1, \mathrm{CC}$ & - & - & $\mathrm{P}$ & $P$ & $P$ & P & $\mathrm{P}$ & $P$ & & & $\mathrm{P}$ & $P$ & - & - & - & & & & & & & \\
\hline $2, \mathrm{CC}$ & & & - & - & - & P & P & $\mathrm{P}$ & & P & $\mathrm{P}$ & $P$ & $\mathrm{P}$ & P & + & - & & & & & & \\
\hline $3, \mathrm{CC}$ & & & - & & & P & $\mathrm{P}$ & $\mathrm{P}$ & $\mathrm{P}$ & $P$ & $\mathrm{P}$ & $P$ & - & & + & $\mathrm{P}$ & - & & & & & \\
\hline $4, \mathrm{CC}$ & & & & & & P & $\mathrm{P}$ & $\mathrm{P}$ & $\mathrm{P}$ & & $\mathrm{P}$ & $\mathrm{P}$ & & $\mathrm{P}$ & + & P & $\mathrm{P}$ & - & & - & - & \\
\hline $5, \mathrm{CC}$ & & & & & & P & & $\mathrm{P}$ & & $P$ & $\mathrm{P}$ & & & $\mathrm{P}$ & $\mathrm{P}$ & P & $\mathrm{P}$ & P & - & + & - & + \\
\hline $6, \mathrm{CC}$ & & & & & & P & & $P$ & & $P$ & $\mathrm{P}$ & & & $\mathbf{P}$ & $\mathrm{P}$ & $\mathrm{P}$ & $\mathrm{P}$ & $\mathrm{P}$ & - & P & P & \\
\hline
\end{tabular}


Table 6. Raw data for Hole 573B (for explanation of symbols see note to this appendix, Table 1).

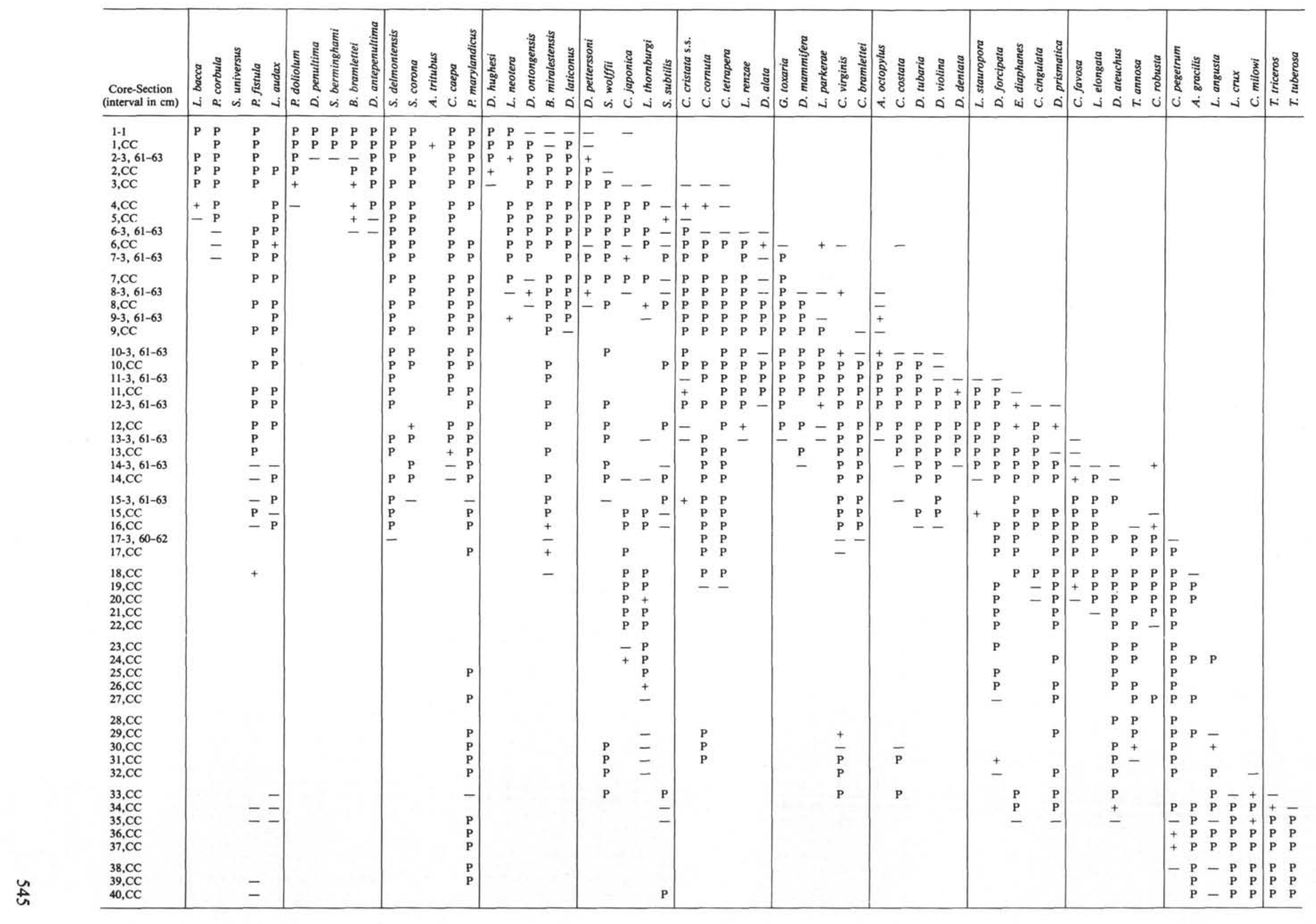


Table 7. Raw data for Hole 574 (for explanation of symbols see note to this appendix, Table 1).

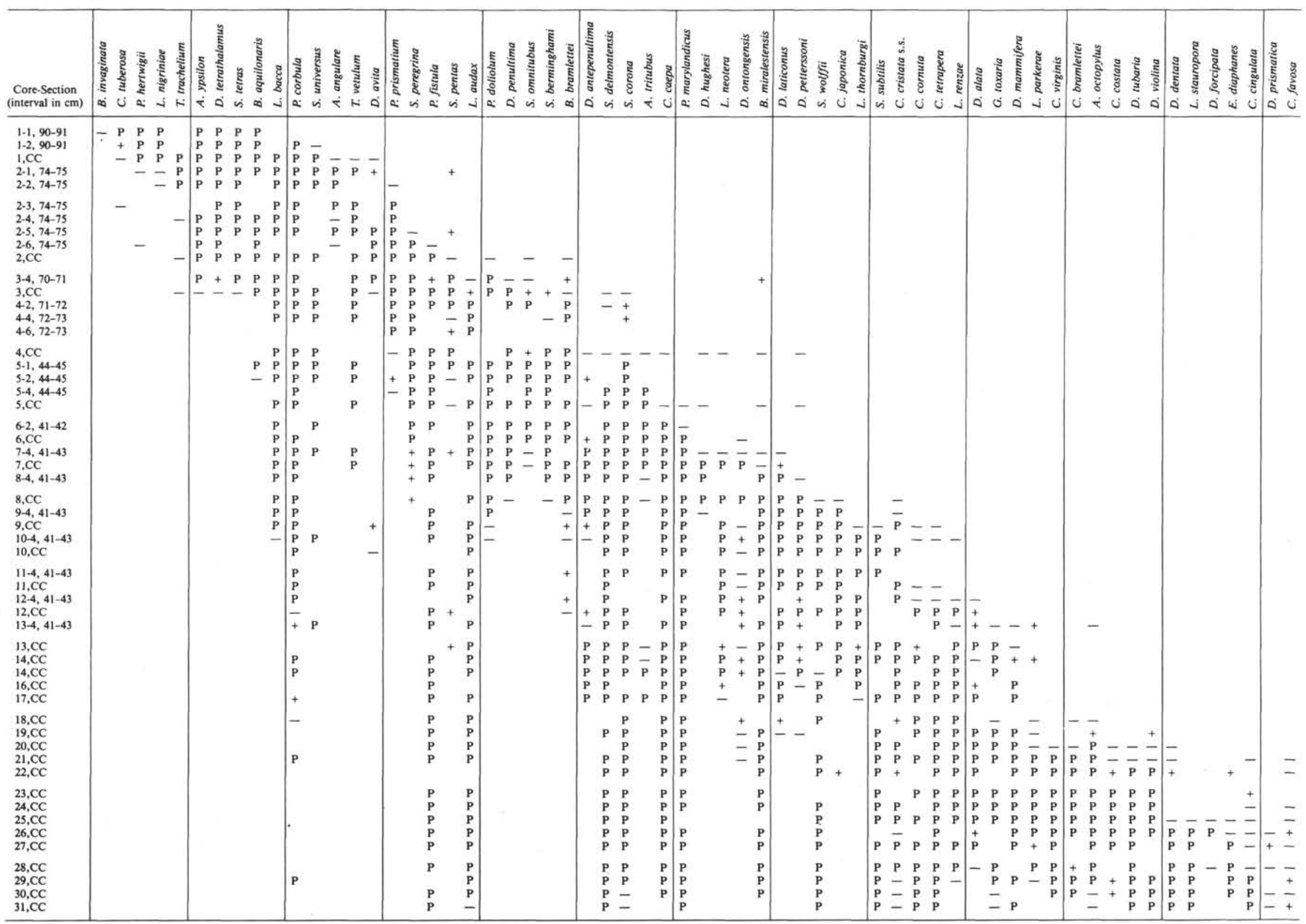


Table 8. Raw data for Hole 574A (for explanation of symbols see note to this appendix, Table 1).

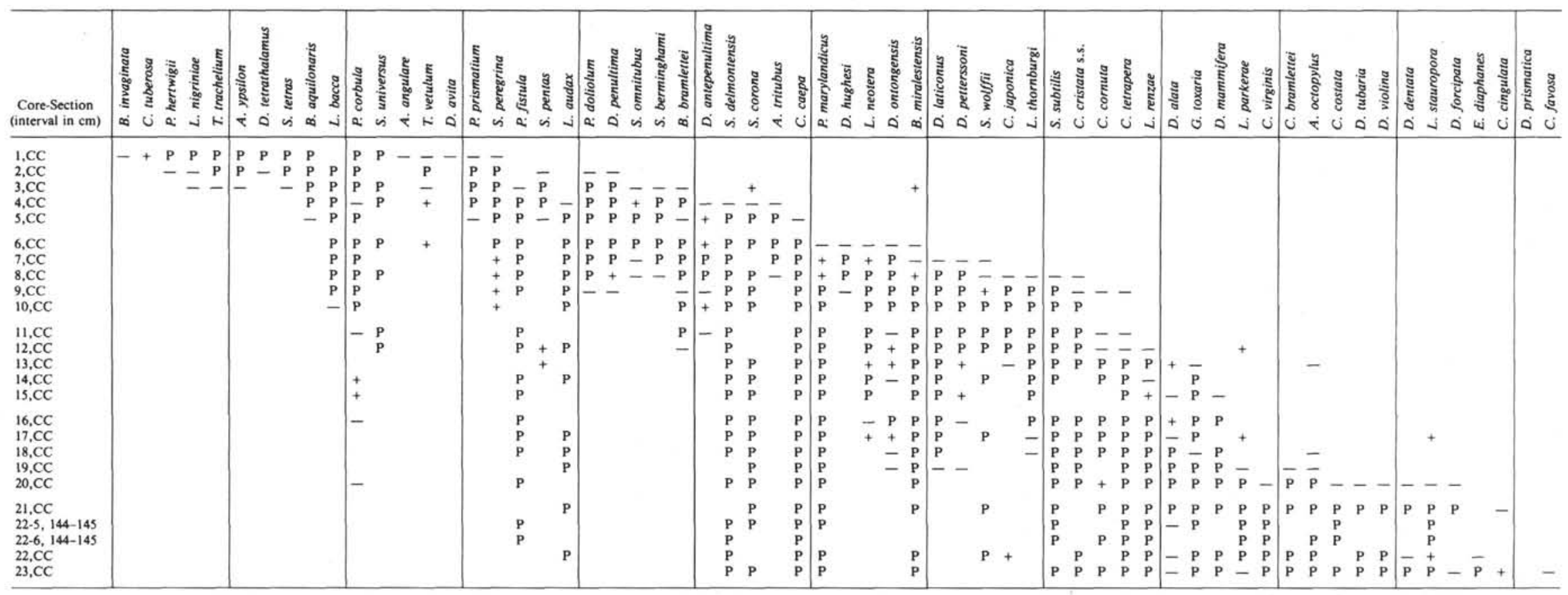


Table 9. Raw data for Hole $574 \mathrm{C}$ (for explanation of symbols see note to this appendix, Table 1).

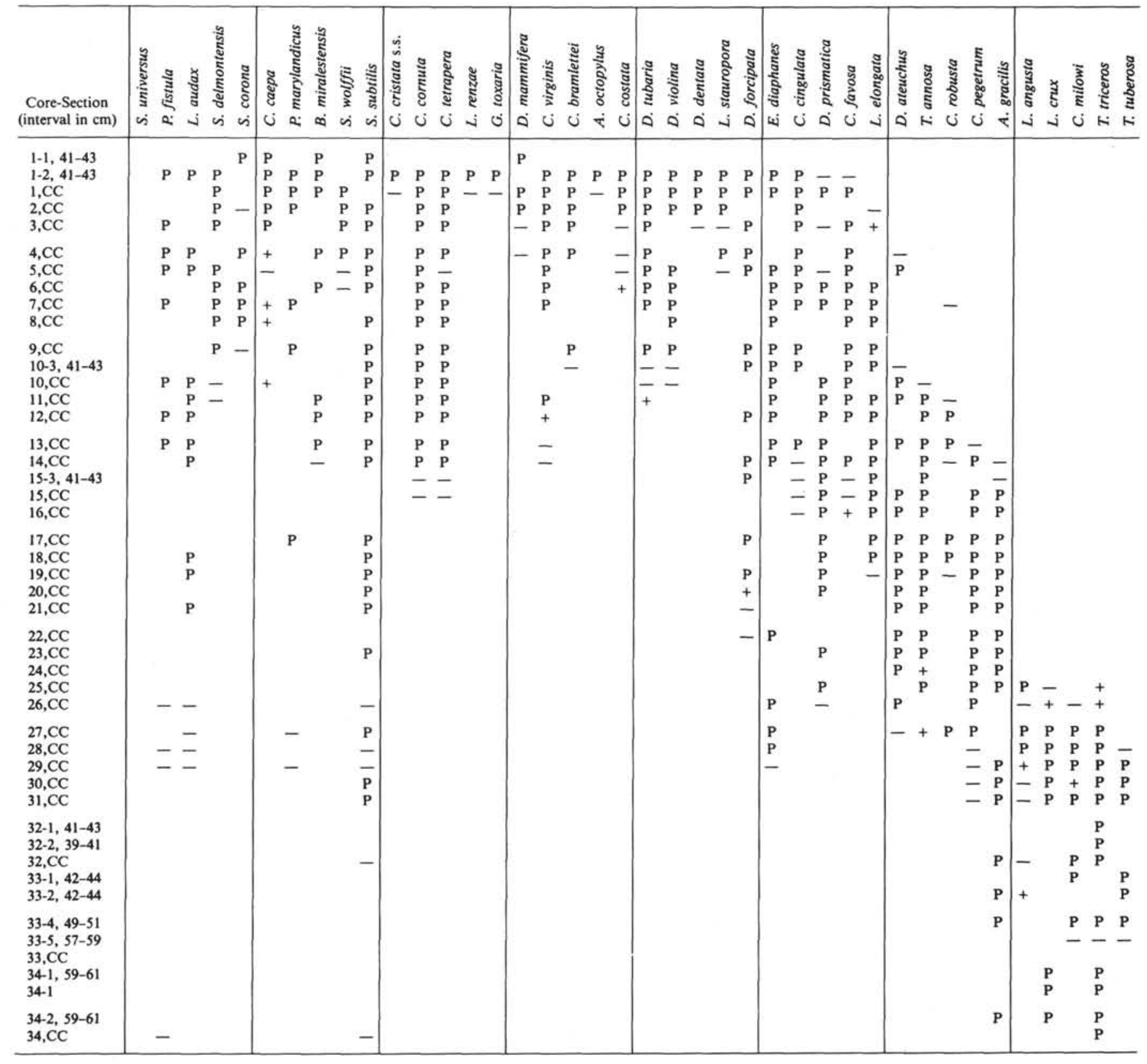


Table 10. Raw data for Hole 575 (for explanation of symbols see note to this appendix, Table 1).

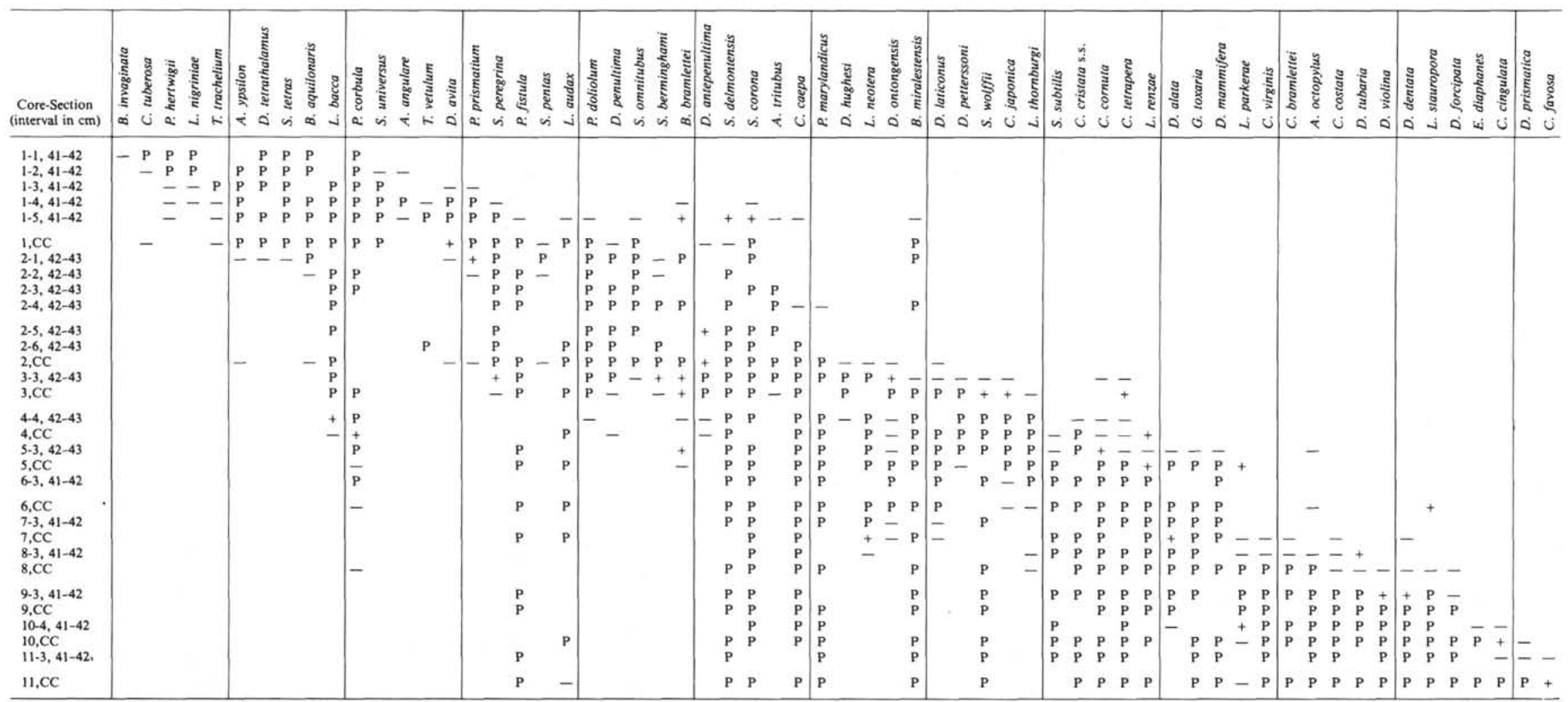


Table 11. Raw data for Hole 572A (for explanation of symbols see note to this appendix, Table 1).

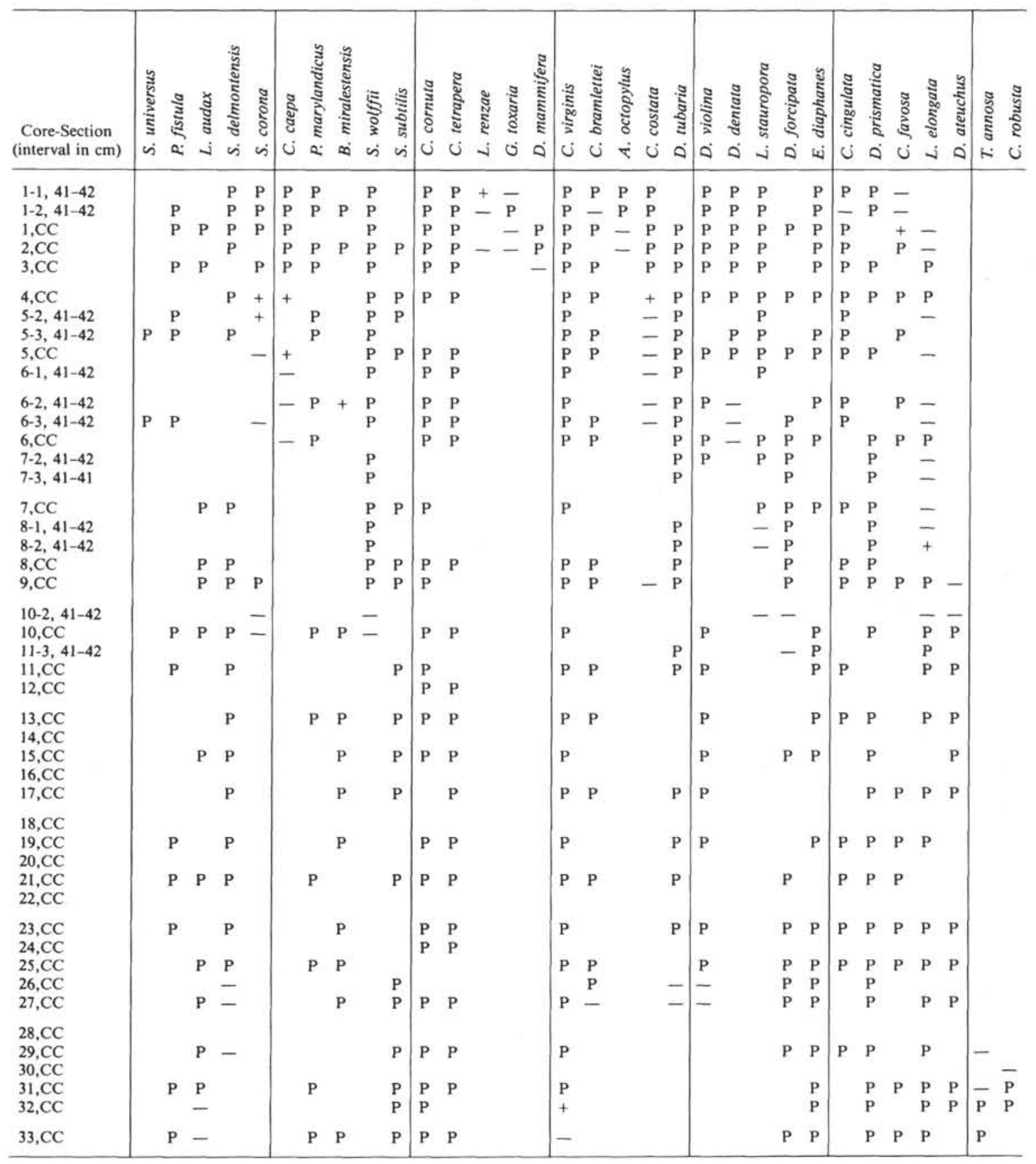


Table 12. Raw data for Hole 575B (for explanation of symbols see note to this appendix, Table 1).

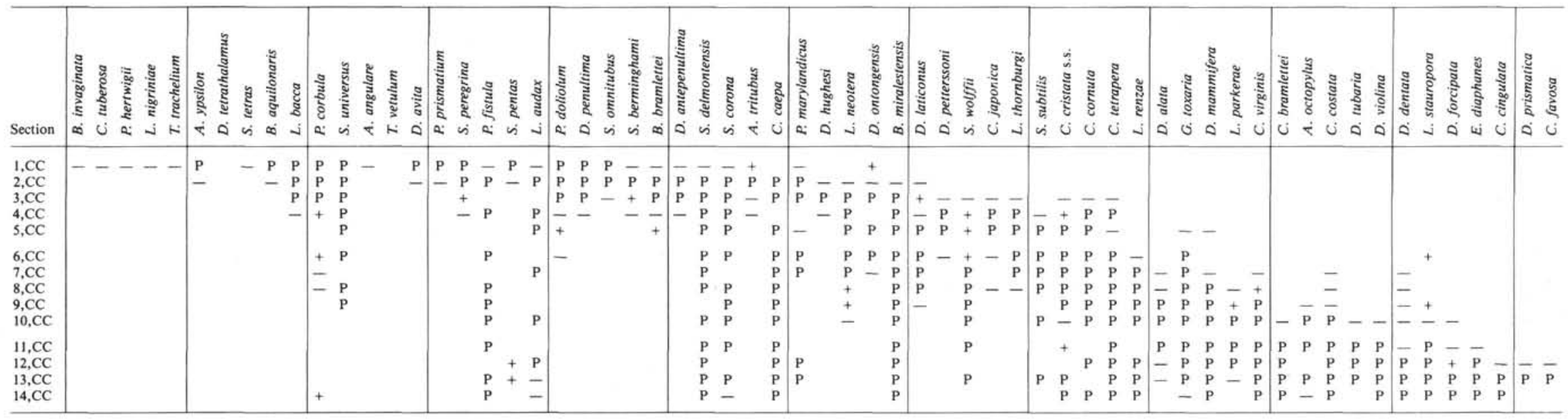

